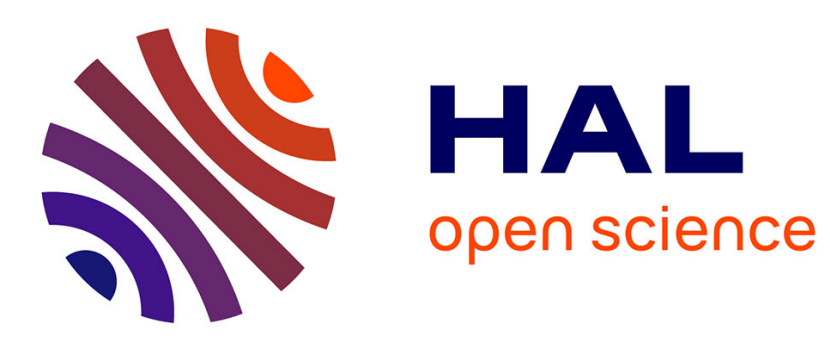

\title{
Generalized Convolution Behaviors and Topological Algebra
}

Henri Bourlès, Ulrich Oberst

\section{To cite this version:}

Henri Bourlès, Ulrich Oberst. Generalized Convolution Behaviors and Topological Algebra. Acta Applicandae Mathematicae, 2015, 141 (1), pp.107 - 148. 10.1007/s10440-015-0007-4 . hal-02368011

\section{HAL Id: hal-02368011 \\ https://hal.science/hal-02368011}

Submitted on 20 Nov 2019

HAL is a multi-disciplinary open access archive for the deposit and dissemination of scientific research documents, whether they are published or not. The documents may come from teaching and research institutions in France or abroad, or from public or private research centers.
L'archive ouverte pluridisciplinaire HAL, est destinée au dépôt et à la diffusion de documents scientifiques de niveau recherche, publiés ou non, émanant des établissements d'enseignement et de recherche français ou étrangers, des laboratoires publics ou privés. 


\title{
Generalized convolution behaviors and topological algebra
}

published online in Acta Appl Math, Springer Verlag, February 5, 2015

DOI 10.1007/s10440-015-0007-4

\author{
Henri Bourlès \\ SATIE, ENS Cachan/CNAM, \\ 61 Avenue President Wilson, F-94230 Cachan, France \\ email: henri.bourles@cnam.fr; tel:+33 140958883 \\ and \\ Ulrich Oberst \\ Institut für Mathematik, Universität Innsbruck \\ Technikerstraße 13, A-6020 Innsbruck, Austria \\ email: Ulrich.Oberst@uibk.ac.at, tel: +435238 53139
}

February 5, 2015

\begin{abstract}
We investigate one-dimensional 'generalized convolution behaviors' (gen. beh.) that comprise differential and delay-differential behaviors in particular. We thus continue work of, for instance, Breth'e, van Eijndhoven, Fliess, Gluesing-Luerssen, Habets, Loiseau, Mounier, Rocha, Vettori, Willems, Yamamoto, Zampieri of the last twenty-five years. The signal space for these behaviors is the space $\mathrm{E}$ of smooth complex-valued functions on the real line. The ring of operators is the commutative integral domain E' of distributions with compact support with its convolution product that acts on $\mathrm{E}$ by a variant of the convolution product and makes it an E'-module. Both E and E' carry their standard topologies. Closed E'-submodules of finite powers of E were introduced and studied by Schwartz already in 1947 under the name 'invariant varieties' and are called gen. beh. here. A gen. beh. is called a behavior if it can be described by finitely many convolution equations. The ring E' is not noetherian and therefore the standard algebraic arguments from one-dimensional differential systems theory have to be completed by methods of topological algebra. Standard constructions like elimination or taking (closed) images of behaviors may lead to gen. beh. and therefore the consideration of the latter is mandatory. It is not known whether all gen. beh. are indeed behaviors, but we show that many of them are, in particular all autonomous ones. The E'-module E is neither injective nor a cogenerator and, in particular, does not admit elimination in Willems' sense. But the signal submodule PE of all polynomial-exponential signals is injective for finitely generated modules and thus admits elimination. This is a useful replacement and approximation of the injectivity of E since the polynomial-exponential part of any gen. beh. is dense in it. We also describe a useful replacement of the cogenerator property and thus establish a strong relation between convolution equations and their solution spaces. Input/output struc-
\end{abstract}


tures of gen. beh. exist and are used to prove that also many nonautonomous generalized behaviors are indeed behaviors. The E'-torsion elements of E, i.e., the smooth functions which satisfy at least one nonzero convolution equation, are called 'mean-periodic functions' and were studied by many outstanding analysts. Their results are significant for gen. beh..

AMS subject classification: 44A35, 46E25, 93B25, 93C 30.

Key words: convolution equation, convolution behavior, elimination, autonomous behavior, input/output structure, duality, topological algebra

\section{Introduction}

The present paper is the elaboration of the research announcement [6].

One-dimensional convolution equations have already a longer history in mathematics and were especially studied in the analytic theory of mean-periodic functions [8], [30], [22], [21], [10], [1], [2]. The paper [2] gives an excellent survey of the theory and its history. The study of such equations and their solution spaces from Willems' behavioral point of view is of more recent origin and was pursued, for instance, by Fliess and Mounier [13], Brethé and Loiseau [7], [23], Glüsing-Lürssen [15], [16], [17], van Eijndhoven and Habets [18], [12], Mounier [25], Rocha and Willems [29], Vettori and Zampieri [32], [33], [34], [35], Yamamoto [36], Yamamoto and Willems [37]. We refer to [16] and [23] for references to the important earlier work on delay-differential equations with commensurate delays.

We continue the work of these colleagues and prove new results on generalized (convolution) behaviors that were introduced and studied by Schwartz already in 1947 as invariant varieties [30, §1]. Variants of these were also considered in the important quoted papers by Eijndhoven and Habets, Glüsing-Lürssen, Vettori and Zampieri. These behaviors are defined as follows: We use the signal space $\mathcal{E}$ of smooth complexvalued functions on the time-axis $\mathbb{R}(\ni t)$ and the algebra $\mathcal{E}^{\prime}$ of distributions with compact support with its convolution product $*$ as ring of operators that acts on $\mathcal{E}^{\prime}$ by a variant $\circ$ of the convolution product $*$ and makes $\mathcal{E}$ an $\mathcal{E}^{\prime}$-module. The ring $\mathcal{E}^{\prime}$ is a commutative integral domain, but not noetherian. Both $\mathcal{E}$ and $\mathcal{E}^{\prime}$ are equipped with their standard topologies. All structures are canonically extended to finite products $\mathcal{E}^{\ell}$ (columns) and $\mathcal{E}^{\prime 1 \times \ell}$ (rows). A generalized behavior is a closed $\mathcal{E}^{\prime}$-submodule $\mathcal{B}$ of some $\mathcal{E}^{\ell}, \ell \in \mathbb{N}$. In connection with the signal submodule $\mathcal{P} \mathcal{E} \subset \mathcal{E}$ of polynomialexponential functions we talk of the generalized $\mathcal{E}^{\prime} \mathcal{E}$-behavior $\mathcal{B}$ or $\mathcal{E}^{\prime} \mathcal{P} \mathcal{E}$-behavior $\mathcal{B} \cap \mathcal{P} \mathcal{E}^{\ell}$. A generalized behavior is called a (convolution) behavior if it admits a kernel representation according to Willems or, in other words, if it can be described by finitely many convolution equations. Convolution equations and (generalized) convolution behaviors are important from the engineering point of view because they comprise differential, delay-differential and integral equations and behaviors. Since the ring $\mathcal{E}^{\prime}$ is not noetherian the standard algebraic arguments from one-dimensional differential systems theory have to be completed by methods of topological algebra. The usual constructions, for instance of elimination or taking (closed) images of convolution behaviors, may lead to generalized behaviors and therefore the consideration of the latter is mandatory. It is not known whether every generalized behavior is indeed a behavior and the proof of Thm. 7.13 suggests that this is probably hard to decide. We show, however, that many generalized behaviors are behaviors, in particular all autonomous ones. In the seminal result [30, Thm. 13 on p. 914, lines before Thm. 13] Schwartz already proved that every generalized behavior $\mathcal{B} \subseteq \mathcal{E}$ is a behavior and defined by two 
equations, but not by one in general. Another important topic of the present paper is the discussion of (Willems') elimination for generalized behaviors.

The paper is organized as follows: Basic facts on generalized $\mathcal{E}^{\prime} \mathcal{E}$-behaviors and complex variables are recalled in Sections 2 and 3. The Paley-Wiener-Schwartz theorem on the Fourier-Laplace transform and one of Ehrenpreis' many important results enable to identify the topological algebra $\mathcal{E}^{\prime}$ with a topological subalgebra (still denoted by $\mathcal{E}^{\prime}$ ) of the ring $\mathcal{O}$ of entire holomorphic functions on $\mathbb{C}$. This is an essential ingredient of the proofs of Thms. 5.8 and 7.13. Localizations of $\mathcal{O}$ and $\mathcal{E}^{\prime}$ with respect to their closed maximal ideals are introduced in Section 3 and are important for the algebraic characterization of topological properties of generalized behaviors in Thm. 4.5 of Section 4. In Thm. 4.4 it is shown that the signal module $\mathcal{E}^{\prime} \mathcal{P} \mathcal{E}$ of polynomialexponential signals is injective for finitely generated modules and thus admits elimination in Willems' sense. In contrast it is known that the $\mathcal{E}^{\prime}$-module $\mathcal{E}$ is not divisible and hence not injective. Since the polynomial-exponential trajectories of a generalized behavior are dense in it (cf. [17, (3.3)], Result 2.1) this property of $\mathcal{P} \mathcal{E}$ is a useful replacement and approximation of the injectivity of $\mathcal{E}$ as module over the ring of differential operators. Since $\operatorname{Hom}_{\mathcal{E}^{\prime}}\left(\mathcal{E}^{\prime} / \mathfrak{a}, \mathcal{E}\right)=0$ for every dense ideal $\mathfrak{a}$ of $\mathcal{E}^{\prime}$ the signal modules $\mathcal{E}^{\prime} \mathcal{E}$ and hence $\mathcal{P} \mathcal{E}$ are not cogenerators, but again Thm. 4.4,(1), describes a useful replacement of the cogenerator property. Section 5 deals with autonomous generalized behaviors that are characterized by the property that the past of each behavior trajectory determines its future. All autonomous generalized behaviors are constructed in Thm. 5.6 and shown to be indeed behaviors in Thm. 5.8. In Section 6 we discuss the characteristic variety, weak controllability and the weakly controllable part of a generalized behavior. Weak controllability of convolution behaviors was introduced and characterized in [17, Thm. 3.12, p.11], for instance. In the last Section 7 we treat input/output structures and the corresponding transfer matrices of generalized behaviors and also the weakly controllable realizations of arbitrary transfer matrices. By means of these notions we show that also many nonautonomous generalized behaviors are indeed behaviors. For instance, a generalized input/output behavior is a behavior if its transfer matrix has an invertible common denominator (Thm. 7.5,(3)) or if it is weakly controllable and has only one input or only one output (Thm. 7.13). Here a distribution $T \in \mathcal{E}^{\prime}$ is called invertible if $T * \mathcal{E}=\mathcal{E}$. In particular, the controllable part of any delay-differential behavior is also a behavior. This solves an open question of $[17$, p. 11]. The principal new results of this paper are Thms. 4.4, 4.5, 5.6, 5.8, 7.5, 7.12 and 7.13 .

We use the following abbreviations: f.d.=finite-dimensional, f.g.=finitely generated, gen. beh.=generalized behavior, $\mathrm{IO}=$ input/output, resp.=respectively.

\section{Basic data}

We are first going to describe the algebra and topology of $\mathcal{E}$ and $\mathcal{E}^{\prime}$ in more precise terms. The space $\mathcal{E}$ is equipped with its strong topology of compact convergence of the functions and all their derivatives [31, p.88]. Its topological dual $\mathcal{E}^{\prime}$ of continuous linear functions $T: \mathcal{E} \rightarrow \mathbb{C}$ is identified with the space of distributions with compact support [31, Thm. XXV]. The space $\mathcal{E}^{\prime}$ carries the weak locally convex topology [3, Ch.II, §6] with respect to the canonical nondegenerate bilinear form

$$
<-,->: \mathcal{E}^{\prime} \times \mathcal{E} \rightarrow \mathbb{C},<T, w>:=T(w),
$$

as does the space $\mathcal{E}$, and the strong topology of uniform convergence on the bounded 
sets of $\mathcal{E}$. Then $\mathcal{E}$ with the strong resp. weak topology is the topological dual of $\mathcal{E}^{\prime}$ with the strong resp. weak topology, i.e., $\mathcal{E}$ with these topologies is reflexive [31, p. 89]. The convolution product $*$ on $\mathcal{E}^{\prime}$, defined by

$$
<S * T, w>=<S_{s}<T_{t}, w(s+t)>>, S, T \in \mathcal{E}^{\prime}, w \in \mathcal{E},
$$

makes $\mathcal{E}^{\prime}$ a commutative (integral) domain and a topological algebra with respect to the strong topology [31, Ch. VI, Thms. IV, VII, XIV]. Also via convolution $\mathcal{E}^{\prime}$ acts on $\mathcal{E}$ and makes it an $\mathcal{E}^{\prime}$-module such that for all $w \in \mathcal{E}$ the map $\mathcal{E}^{\prime} \rightarrow \mathcal{E}, T \mapsto T * w$, is continuous with respect to both topologies. We define the action $\circ$ by

$$
\circ: \mathcal{E}^{\prime} \times \mathcal{E} \rightarrow \mathcal{E},\left\langle S, T \circ w>=<S * T, w>, S, T \in \mathcal{E}^{\prime}, w \in \mathcal{E} .\right.
$$

The action $\circ$ is related to the convolution product via the algebra involution $T \mapsto \check{T}$ of $\mathcal{E}^{\prime}$, i.e., by

$$
T \circ w=\check{T} * w \text { where }<\check{T}_{t}, w(t)>:=<T_{t}, w(-t)>.
$$

In particular, $\mathcal{E}$ with $\circ$ is also an $\mathcal{E}^{\prime}$-module. In the sequel we will use $\mathcal{E}^{\prime}$ as ring of operators and $\mathcal{E}^{\prime} \mathcal{E}$ with the action $\circ$ as signal module. This signal space and module structure $\circ$ are important because various cases of engineering significance, in particular differential, delay-differential and integral linear equations are included, for instance

$$
\begin{gathered}
\left(\delta_{h} \circ w\right)(t)=w(t+h), t, h \in \mathbb{R},<\delta_{h}, w>:=w(h), \\
\left(\left(\delta_{1}-\delta_{0}\right) \circ w\right)(t)=w(t+1)-w(t), \\
\delta:=\delta_{0},-\delta^{\prime} \circ w=\delta^{\prime} * w=w^{\prime}, \\
(T \circ w)(t)=(\check{T} * w)(t)=\int_{-\infty}^{\infty} T(x-t) w(x) d x \text { for } T \in \\
\mathcal{L}_{0}^{1}(\mathbb{R}, \mathbb{C}):=\{\text { integrable functions with compact support }\} .
\end{gathered}
$$

The preceding notions and results are canonically extended to the finite powers $\mathcal{E}^{\ell}:=\mathcal{E}^{\ell \times 1}$ (columns) resp. $\mathcal{E}^{\prime 1 \times \ell}$ (rows) for any $\ell \in \mathbb{N}$. The closed subspaces of $\mathcal{E}^{\ell}$ with respect to the strong or the weak topologies coincide [3, §II.6.3, Cor. 3]. If $R \in \mathcal{E}^{\prime k \times \ell}$ and $w \in \mathcal{E}^{\ell}$ then $R \circ w \in \mathcal{E}^{k}$ is defined and homogeneous linear systems $R \circ w=0$ and inhomogeneous systems $R \circ w=u$ can and will be considered. A closed subspace $\mathcal{B} \subseteq \mathcal{E}^{\ell}$ is translation-invariant, i.e., $\delta_{h} \circ \mathcal{B}=\delta_{h} * \mathcal{B}=\mathcal{B}$ for all $h \in \mathbb{R}$, if and only if it is an $\mathcal{E}^{\prime}$-submodule of $\mathcal{E}^{\ell}[31,(\mathrm{VI}, 3 ; 16)]$. The closed $\mathcal{E}^{\prime}$-submodules of some $\mathcal{E}^{\ell}, \ell \in \mathbb{N}$, are the generalized (convolution) behaviors introduced above. Recently Lomadze characterized the differential behaviors among the gen. beh.. A (convolution) behavior is a gen. beh. of the form

$$
\begin{gathered}
\mathcal{B}:=\left\{w \in \mathcal{E}^{\ell} ; R \circ w=0\right\}= \\
\left\{w \in \mathcal{E}^{\ell} ; \forall i=1, \cdots, k: \sum_{j=1}^{\ell} R_{i j} \circ w_{j}=0\right\}, R \in \mathcal{E}^{\prime k \times \ell},
\end{gathered}
$$

that obviously is a closed $\mathcal{E}^{\prime}$-submodule of $\mathcal{E}^{\ell}$ and the solution space of finitely many convolution equations. The representation in (6) is Willems' kernel representation of the behavior. 
We define the polar or orthogonal submodule of an $\mathcal{E}^{\prime}$-submodule $U \subseteq \mathcal{E}^{\prime 1 \times \ell}$ as [34, $\S 2],[4$, Thm. 2.23]

$$
U^{o}:=\left\{w \in \mathcal{E}^{\ell} ;\langle U, w>=0\}=U^{\perp}:=\left\{w \in \mathcal{E}^{\ell} ; U \circ w=0\right\} \subseteq \mathcal{E}^{\ell}\right.
$$

and likewise, for an $\mathcal{E}^{\prime}$-submodule $\mathcal{B} \subseteq \mathcal{E}^{\ell}$,

$$
\mathcal{B}^{o}:=\left\{\xi \in \mathcal{E}^{\prime 1 \times \ell} ;<\xi, \mathcal{B}>=0\right\}=\mathcal{B}^{\perp}:=\left\{\xi \in \mathcal{E}^{\prime 1 \times \ell} ; \xi \circ \mathcal{B}=0\right\} .
$$

The submodule $U^{\perp}$ is obviously closed and therefore a gen. beh. and likewise $\mathcal{B}^{\perp}$ is closed. The maps $U \mapsto U^{\circ}=U^{\perp}$ and $\mathcal{B} \mapsto \mathcal{B}^{\circ}=\mathcal{B}^{\perp}$ form a Galois correspondence, i.e., are order-reversing and satisfy

$$
U \subseteq U^{\perp \perp}, \mathcal{B} \subseteq \mathcal{B}^{\perp \perp}, U^{\perp}=U^{\perp \perp \perp}, \mathcal{B}^{\perp}=\mathcal{B}^{\perp \perp \perp}
$$

Let $\mathrm{cl}_{E}(U)$ resp. $\operatorname{cl}_{E}(\mathcal{B})$ denote the closures of $U$ resp. $\mathcal{B}$ in $\mathcal{E}^{\prime 1 \times \ell}$ resp. $\mathcal{E}^{\ell}$. The important bipolar theorem [3, Thm. II.6.1] implies

$$
\mathrm{cl}_{E}(U)=U^{o o}=U^{\perp \perp} \text { and } \operatorname{cl}_{E}(\mathcal{B})=\mathcal{B}^{o o}=\mathcal{B}^{\perp \perp} .
$$

Hence the Galois correspondence establishes a one-one correspondence, also called polarity or duality, between closed submodules $U \subseteq \mathcal{E}^{\prime 1 \times \ell}$ and gen. beh. $\mathcal{B}:=U^{\perp} \subseteq$ $\mathcal{E}^{\ell}$. In other words, $U$ resp. $\mathcal{B}$ are closed if and only if $U=U^{\perp \perp}$ resp. $\mathcal{B}=\mathcal{B}^{\perp \perp}$. For a matrix $R \in \mathcal{E}^{\prime k \times \ell}$ the corresponding behavior is obtained as

$$
\left\{w \in \mathcal{E}^{\ell} ; R \circ w=0\right\}=U^{\perp} \text { where } U:=\mathcal{E}^{\prime 1 \times k} R=\sum_{i=1}^{k} \mathcal{E}^{\prime} R_{i-} \subseteq \mathcal{E}^{\prime 1 \times \ell}
$$

is the row module of $R$. An arbitrary gen. beh. $\mathcal{B}=U^{\perp}$ is a behavior if and only if there are $k \in \mathbb{N}$ and $R \in \mathcal{E}^{\prime k \times \ell}$ such that

$$
\mathcal{B}=U^{\perp}=\left(\mathcal{E}^{\prime 1 \times k} R\right)^{\perp} \text { or, equivalently, } \mathcal{B}^{\perp}=\operatorname{cl}_{E}(U)=\operatorname{cl}_{E}\left(\mathcal{E}^{\prime 1 \times k} R\right),
$$

i.e., if the closure of $U$ contains a dense f.g. submodule. Schwartz' seminal result [30, Thm. 13 on p. 914] thus signifies that every closed ideal $\mathfrak{a}$ of $\mathcal{E}^{\prime}$ contains a dense ideal $\mathcal{E}^{\prime} T_{1}+\mathcal{E}^{\prime} T_{2}, T_{i} \in \mathcal{E}^{\prime}$. In general, a does not contain a dense principal ideal, i.e., the behavior $\mathfrak{a}^{\perp}$ cannot be described by one equation or, in general terms, by a matrix with linearly independent rows (=of maximal row rank), cf. [30, lines before Thm. 13 on p.914]. Therefore the conjecture [17, (2) on p.12] concerning delay-differential behaviors is false for arbitrary convolution behaviors.

For any $\mathcal{E}^{\prime}$-module $U \subseteq \mathcal{E}^{1 \times \ell}$ we also introduce the factor module $M=\mathcal{E}^{\prime 1 \times \ell} / U$ with its factor or identification topology. The module $M$ is separated or a Hausdorff space if and only if $U$ is closed. As for differential systems the canonical Malgrange isomorphism

$$
\begin{gathered}
\operatorname{Hom}_{\mathcal{E}^{\prime}}\left(\mathcal{E}^{\prime 1 \times \ell} / U, \mathcal{E}\right)=\operatorname{Hom}_{\mathcal{E}^{\prime}}\left(\mathcal{E}^{\prime 1 \times \ell} / \operatorname{cl}_{E}(U), \mathcal{E}\right) \cong \\
\mathcal{B}:=U^{\perp}=\operatorname{cl}_{E}(U)^{\perp}, \varphi \leftrightarrow w, \varphi(\xi+U)=\xi \circ w, \xi \in \mathcal{E}^{\prime 1 \times \ell},
\end{gathered}
$$

holds. We consider this as an algebraic isomorphism only and not as a topological one. The left exact functor $\operatorname{Hom}_{\mathcal{E}^{\prime}}(-, \mathcal{E})$ on f.g. $\mathcal{E}^{\prime}$-modules thus is an equally important tool for generalized convolution behaviors as it is for differential behaviors.

We are now going to describe the space $\mathcal{P E} \subseteq \mathcal{E}$ of polynomial-exponential functions 
in more detail. From (5) we know $-\delta^{\prime} \circ w=w^{\prime}=\frac{d w}{d t}$. We therefore identify the differential operator $\partial:=\frac{d}{d t}$ with its corresponding distribution $-\delta^{\prime}, \partial:=-\delta^{\prime}$, and conclude that the polynomial algebra $\mathbb{C}[\partial]=\mathbb{C}\left[-\delta^{\prime}\right]$ is the subalgebra of $\mathcal{E}^{\prime}$ of linear differential operators with constant coefficients. If $N$ is any module over a commutative integral domain $A$ its torsion submodule $\operatorname{tor}(N) \subseteq N$ is the submodule of all elements $x$ for which there is a nonzero $a \in A$ that annihilates $x$, i.e., $a x=0$. If $\operatorname{tor}(N)=N$ resp. $\operatorname{tor}(N)=0$ the module $N$ is called torsion or a torsion module resp. torsionfree. From standard one-dimensional differential systems theory it is known that $\mathcal{P} \mathcal{E}$ is the torsion submodule of $\mathcal{E}$ as $\mathbb{C}[\partial]=\mathbb{C}\left[\frac{d}{d t}\right]$-module. Since $\mathbb{C}[\partial]$ is a principal ideal domain with the representative system of prime elements $\partial-z, z \in \mathbb{C}$, any torsion module admits the canonical primary direct sum decomposition. Recall the definition of direct sums here: If $\left(V_{i}\right)_{i \in I}$ is a possibly infinite family of submodules of a module $V$ the sum $\sum_{i \in I} V_{i}$ is the least submodule of $V$ containing all $V_{i}$ and consists of all sums $\sum_{i \in I} x_{i}$ where $x_{i} \in V_{i}$ and only finitely many $x_{i}$ are nonzero or, in other words, almost all $x_{i}$ are zero. Without this condition the sum $\sum_{i} x_{i}$ does not make sense in pure algebra; convergence is not considered here. The sum $\sum_{i} V_{i}$ is called direct and then written as $\bigoplus_{i \in I} V_{i}$ if every $x \in \sum_{i} V_{i}$ has a unique representation $x=\sum_{i} x_{i}$. For $\mathcal{P} \mathcal{E}$ we now obtain:

$$
\begin{gathered}
\mathcal{P \mathcal { E }}:=\operatorname{tor}(\mathbb{C}[\partial] \mathcal{E})=\{w \in \mathcal{E} ; \exists 0 \neq T \in \mathbb{C}[\partial] \text { with } T \circ w=0\}=\bigoplus_{z \in \mathbb{C}} \mathcal{P} \mathcal{E}(z), \\
\text { where } \mathcal{P} \mathcal{E}(z):=\bigcup_{k \in \mathbb{N}} \operatorname{ann}_{\mathcal{E}}\left((\partial-z)^{k}\right), \\
\operatorname{ann}_{\mathcal{E}}\left((\partial-z)^{k}\right):=\left(\mathcal{E}^{\prime}(\partial-z)^{k}\right)^{\perp}=\left\{w \in \mathcal{E} ;(\partial-z)^{k} \circ w=0\right\}= \\
\mathbb{C}[t]_{<k} e^{z t}=\bigoplus_{j=0}^{k-1} \mathbb{C} e_{z, j}, \mathbb{C}[t]_{<k}:=\bigoplus_{j=0}^{k-1} \mathbb{C} t^{j}, \\
e_{z, j}:=\frac{t^{j}}{j !} e^{z t},(\partial-z)^{m} \circ e_{z, j}= \begin{cases}e_{z, j-m} & \text { if } m \leq j \\
0 & \text { if } m>j \\
\mathcal{P} \mathcal{E}(z)=\mathbb{C}[t] e^{z t}, \mathcal{P} \mathcal{E}=\bigoplus_{z \in \mathbb{C}} \mathbb{C}[t] e^{z t} .\end{cases}
\end{gathered}
$$

For a submodule $U \subseteq \mathcal{E}^{1 \times \ell}$ and its behavior $\mathcal{B}:=U^{\perp}$ this implies the decomposition

$$
\begin{aligned}
& \mathcal{B} \bigcap \mathcal{P} \mathcal{E}^{\ell}=\left\{w=\sum_{z \in \mathbb{C}} w_{z}\right.\left.\in \bigoplus_{z \in \mathbb{C}} \mathcal{P} \mathcal{E}(z)^{\ell} ; U \circ \sum_{z \in \mathbb{C}} w_{z}=\sum_{z \in \mathbb{C}} U \circ w_{z}=0\right\}= \\
& \bigoplus_{z \in \mathbb{C}}\left(\mathcal{B} \bigcap \mathcal{P} \mathcal{E}(z)^{\ell}\right) .
\end{aligned}
$$

Since the ring $\mathcal{E}^{\prime}$ is commutative the annihilators $\operatorname{ann}_{\mathcal{E}}\left((\partial-z)^{k}\right)$ are $\mathbb{C}$-f.d. $\mathcal{E}^{\prime}$ submodules of $\mathcal{E}$ and indeed behaviors and therefore also the $\mathcal{P} \mathcal{E}(z)=\mathbb{C}[t] e^{z t}$ and $\mathcal{P} \mathcal{E}=\oplus_{z \in \mathbb{C}} \mathcal{P} \mathcal{E}(z)$ are $\mathcal{E}^{\prime}$-submodules of $\mathcal{E}$, but not closed. The isomorphism (13) induces the isomorphism

$$
\operatorname{Hom}_{\mathcal{E}^{\prime}}\left(\mathcal{E}^{\prime 1 \times \ell} / U, \mathcal{P} \mathcal{E}\right) \cong \mathcal{B} \bigcap \mathcal{P} \mathcal{E}^{\ell}
$$

for the polynomial-exponential part of $\mathcal{B}$. The following essential result was the fundamental result Thm. 6 of [30] for $\mathcal{B} \subseteq \mathcal{E}$ and was extended to arbitrary gen. beh. in $[17,(3.3)]$ by means of [27]. 
Result 2.1. The polynomial-exponential part $\mathcal{B} \cap \mathcal{P} \mathcal{E}^{\ell}$ of a gen. beh. $\mathcal{B} \subseteq \mathcal{E}^{\ell}$ is dense in $\mathcal{B}$, hence

$$
\mathcal{B}^{\perp}=\mathcal{B}^{o}=\left(\mathcal{B} \bigcap \mathcal{P} \mathcal{E}^{\ell}\right)^{o}=\left(\mathcal{B} \bigcap \mathcal{P} \mathcal{E}^{\ell}\right)^{\perp}
$$

This is false in higher dimensions and therefore the theory of this paper cannot be extended to higher dimensions.

\section{The use of complex variables}

Let $\mathcal{O}:=\mathcal{O}(\mathbb{C})$ denote the $\mathbb{C}$-algebra of entire functions (everywhere convergent power series) in the complex variable $s \in \mathbb{C}$. It is a Fréchet algebra with the topology of compact convergence. The Fourier transform or Fourier-Laplace transform [19, Thm. 7.1.14]

$$
\mathcal{F}: \mathcal{E}^{\prime} \rightarrow \mathcal{O}, T \mapsto \widehat{T}, \widehat{T}(s):=<T_{t}, e^{-i t s}>,
$$

is an injective algebra homomorphism where $\mathcal{E}^{\prime}$ resp. $\mathcal{O}$ are furnished with the convolution resp. with the pointwise multiplication. For $a>0, p \in \mathbb{N}$ and $F \in \mathcal{O}$ define

$$
\begin{gathered}
\|F\|_{a, p}:=\sup _{z \in \mathbb{C}}|F(z)|(1+|z|)^{-p} e^{-a|\Im(z)|}, \Im(z):=\text { imaginary part of } z, \\
\mathcal{O}_{a, p}:=\left\{F \in \mathcal{O} ;\|F\|_{a, p}<\infty\right\}= \\
\left\{F \in \mathcal{O} ; \exists C>0 \forall z \in \mathbb{C}:|F(z)| \leq C(1+|z|)^{p} e^{a|\Im(z)|}\right\}, \\
P W S:=\bigcup_{a>0, p \in \mathbb{N}} \mathcal{O}_{a, p}, \mathcal{O}_{a, p} \subseteq \mathcal{O}_{a^{\prime}, p^{\prime}} \text { if } a \leq a^{\prime}, p \leq p^{\prime}, \\
\mathcal{O}_{a, p} \subsetneq \mathcal{O}_{a^{\prime}, p} \text { if } a<a^{\prime}, \mathcal{O}_{a, p} \subsetneq \mathcal{O}_{a, p^{\prime}} \text { if } p<p^{\prime} .
\end{gathered}
$$

Then $\mathcal{O}_{a, p}$ is a Banach space with the norm $\|-\|_{a, p}$ and a convergent sequence in $\mathcal{O}_{a, p}$ is compactly convergent in particular. The Paley-Wiener-Schwartz theorem [19, Thm. 7.3.1] implies the algebra isomorphism

$$
\begin{gathered}
\mathcal{F}: \mathcal{E}^{\prime} \cong P W S, T \mapsto \widehat{T} \\
\operatorname{supp}(T) \subseteq[-a, a]:=\{t \in \mathbb{R} ;|t| \leq a\} \Longleftrightarrow \widehat{T} \in \bigcup_{p \in \mathbb{N}} \mathcal{O}_{a, p}
\end{gathered}
$$

where $\operatorname{supp}(T)$ is the support of $T$ as distribution. This theorem was an important tool already in [33], [17] and [12].

A sequence $\left(T_{n}\right)_{n \in \mathbb{N}} \in \mathcal{E}^{\prime \mathbb{N}}$ converges weakly (and then also strongly) to $T \in \mathcal{E}^{\prime}$ if and only if $\lim _{n \rightarrow \infty}<T_{n}, w>=<T, w>$ for all $w \in \mathcal{E}$. According to [11, Lemma 5.17, p.155], [2, p.211] this is equivalent to the existence of

$$
\begin{gathered}
a>0, p \in \mathbb{N} \text { such that } \forall n \in \mathbb{N}: \widehat{T_{n}}, \widehat{T} \in \mathcal{O}_{a, p} \text { and } \\
\qquad \lim _{n \rightarrow \infty}\left\|\widehat{T_{n}}-\widehat{T}\right\|_{a, p}=0 .
\end{gathered}
$$

This enables the construction of distributions with support in $[-a, a]$ by limit processes in the Banach space $\mathcal{O}_{a, p}$ and is very important for the present paper. In the sequel we identify

$$
\begin{gathered}
\mathcal{E}^{\prime}=P W S \subset \mathcal{O}, T=\widehat{T}, T(s):=\widehat{T}(s)=<T_{t}, e^{-i t s}>, \widehat{\delta_{h}}=e^{-i h s}, h \in \mathbb{R}, \\
\partial=-\delta^{\prime}=\widehat{-\delta^{\prime}}=<-\delta^{\prime}, e^{-i s t}>=(-1)(-1)(-i s)=-i s, s=i \partial .
\end{gathered}
$$


Hence $\mathbb{C}[s]=\mathbb{C}[i \partial]=\mathbb{C}[\partial]=\mathbb{C}\left[\delta^{\prime}\right] \subset \mathcal{E}^{\prime}$ is the subalgebra of differential operators. The algebra $\mathcal{O}$ is a Stein algebra $[5, \S 6]$ and has many valuable algebraic properties. An ideal $\mathfrak{b}$ of $\mathcal{O}$ is closed if and only if it is f.g. and then even a principal ideal, hence $\mathcal{O}$ is a Bézout domain and therefore also coherent [5, Def. and Cor. 2.3]. More generally, submodules $U \subseteq \mathcal{O}^{1 \times \ell}$ are closed if and only if they are f.g. and then indeed free. This implies that the f.g. Stein modules $\mathcal{O}^{1 \times \ell} / U, U$ closed, [5, Result 6.1, Thm. 6.2] are precisely the finitely presented or coherent ones. The principal ideals $\mathcal{E}^{\prime} f, 0 \neq f \in \mathcal{E}^{\prime}$, of $\mathcal{E}^{\prime}$ are not closed in general. Indeed, the following equivalences hold [20, Thms. 16.3.10, 16.5.7, Def. 16.3.12 ] (cf. (53)):

$$
\mathcal{E}^{\prime} f \text { is closed } \Longleftrightarrow \mathcal{E}^{\prime} f=\mathcal{E}^{\prime} \bigcap \mathcal{O} f \Longleftrightarrow f \circ \mathcal{E}=f * \mathcal{E}=\mathcal{E}
$$

Then $f$ is called invertible. Smooth functions with compact support, considered as distributions, are never invertible whereas all differential operators $f \in \mathbb{C}[s]=\mathbb{C}[\partial] \subset$ $\mathcal{E}^{\prime}$ are invertible due to the standard result $f \circ \mathcal{E}=\mathcal{E}$. This applies especially to the prime powers $(s-z)^{k}=(i \partial-z)^{k}=i^{k}(\partial+i z)^{k}$ and was widely exploited in the seminal paper [30]. The ideals $\mathfrak{m}_{O}(z)=\mathcal{O}(s-z), z \in \mathbb{C}$, are precisely the closed maximal ideals of $\mathcal{O}$. For every $z \in \mathbb{C}$ the $\operatorname{ring} \mathcal{O}_{z}:=\mathbb{C}\langle s-z\rangle$ is the ring of locally convergent power series at $z$. It is a discrete valuation ring (DVR) (cf. [24, §11]), i.e., a principal ideal domain with the unique (up to association) prime element $s-z$ and unique maximal ideal $\mathfrak{m}_{z}=\mathcal{O}_{z}(s-z)$. The identity theorem implies the inclusion $\mathcal{O} \subset \mathcal{O}_{z}, f=f_{z}:=\sum_{n=0}^{\infty} f^{(n)}(z)(n !)^{-1}(s-z)^{n}$. The closure of an $\mathcal{O}$-submodule $V \subseteq \mathcal{O}^{1 \times \ell}$ is

$$
\operatorname{cl}_{O}(V)=\left\{w \in \mathcal{O}^{1 \times \ell} ; \forall z \in \mathbb{C}: w_{z} \in \mathcal{O}_{z} V \subseteq \mathcal{O}_{z}^{1 \times \ell}\right\}
$$

[5, Result 6.1,(8)]. Equations (22) and (14) imply

$$
\begin{gathered}
\mathcal{E}^{\prime}(s-z)^{k}=\mathcal{E}^{\prime}(\partial+i z)^{k}=\mathcal{E}^{\prime} \bigcap \mathcal{O}(s-z)^{k} \text { for all } k \in \mathbb{N} \text { and } \\
\operatorname{ann}_{\mathcal{E}}\left((s-z)^{k}\right)=\left\{w \in \mathcal{E} ;(\partial+i z)^{k} \circ w=0\right\}=\mathbb{C}[t]_{<k} e^{-i z t} .
\end{gathered}
$$

There results the chain of maximal ideals with residue field $\mathbb{C}$

$$
\begin{gathered}
\mathfrak{m}_{z} \supset \mathfrak{m}_{O}(z):=\mathcal{O} \bigcap \mathfrak{m}_{z}=\{f \in \mathcal{O} ; f(z)=0\}= \\
\mathcal{O}(s-z) \supset \mathfrak{m}_{E}(z):=\mathcal{E}^{\prime} \bigcap \mathfrak{m}_{O}(z)=\mathcal{E}^{\prime}(s-z)=\mathcal{E}^{\prime}(\partial+i z) \supset \\
\mathfrak{m}_{P}(z):=\mathbb{C}[s] \bigcap \mathfrak{m}_{O}(z)=\mathbb{C}[s](s-z)=\mathbb{C}[\partial](\partial+i z) .
\end{gathered}
$$

The maximal ideals induce the local quotient rings

$$
\begin{gathered}
\mathcal{O}_{z} \supset \mathcal{O}_{\mathfrak{m}_{O}(z)} \supset \mathcal{E}_{\mathfrak{m}_{E}(z)}^{\prime}:=\left\{f g^{-1} ; f, g \in \mathcal{E}^{\prime}, g \in \mathcal{E}^{\prime} \backslash \mathfrak{m}_{E}(z)\right\}= \\
\left\{f g^{-1} ; f, g \in \mathcal{E}^{\prime}, g(z) \neq 0\right\} \supset \mathbb{C}[s]_{\mathfrak{m}_{P}(z)}
\end{gathered}
$$

Lemma 3.1. All local rings in (26) are DVRs with the unique prime element $s-z$ or $\partial+i z$, up to association (units).

Proof. For $\mathcal{O}_{z}=\mathbb{C}\langle s-z\rangle, \mathcal{O}_{\mathfrak{m}_{O}(z)}$ and $\mathbb{C}[s]_{\mathfrak{m}_{P}(z)}$ this is a standard result: For all three local rings $A$ with maximal ideal $\mathfrak{m}$ the value $u(z)=u+\mathfrak{m} \in A / \mathfrak{m} \underset{\text { ident. }}{=} \mathbb{C}$ of a ring element $u \in A$ is defined and $u$ is a unit if and only if $u(z) \neq 0$. One represents an arbitrary nonzero element $h$ of the ring in the unique form $h=u(s-z)^{k}$ where 
$u(z) \neq 0$ and hence $u$ is a unit and $k$ is the multiplicity. This implies the DVR property. For $A=\mathcal{E}_{\mathfrak{m}_{E}(z)}^{\prime}$ the proof is a variant of that for $\mathcal{O}_{\mathfrak{m}_{O}(z)}$ : Again it suffices to show that each element $h$ of this ring has a unique representation $h=u(s-z)^{k}$ with a unit $u$ of $\mathcal{E}_{\mathfrak{m}_{E}(z)}^{\prime}$. But let

$$
\begin{gathered}
h=f g^{-1}, f, g \in \mathcal{E}^{\prime}, g(z) \neq 0, \text { and } f=f_{1}(s-z)^{k}, f_{1} \in \mathcal{O}, f_{1}(z) \neq 0 \Longrightarrow \\
h=\left(f_{1} g^{-1}\right)(s-z)^{k} .
\end{gathered}
$$

From (22) we infer $f_{1} \in \mathcal{E}^{\prime}$ and from $g(z) \neq 0, f_{1}(z) \neq 0$ or $g, f_{1} \in \mathcal{E}^{\prime} \backslash \mathfrak{m}_{E}(z)$ that $u:=f_{1} g^{-1}$ is a unit of $\mathcal{E}_{\mathfrak{m}_{E}(z)}^{\prime}$.

The representation $h=u(s-z)^{k}$ is unique since it is unique in $\mathcal{O}_{z}=\mathbb{C}\langle s-z\rangle$.

By means of (24) we also conclude

$$
\begin{gathered}
\mathfrak{m}_{z}^{k}=\mathcal{O}_{z}(s-z)^{k} \supset \mathfrak{m}_{O}(z)^{k}:=\mathcal{O} \bigcap \mathfrak{m}_{z}^{k}=\mathcal{O}(s-z)^{k} \supset \\
\mathfrak{m}_{E}(z)^{k}:=\mathcal{E}^{\prime} \bigcap \mathfrak{m}_{z}^{k}=\mathcal{E}^{\prime}(s-z)^{k} \supset \mathfrak{m}_{P}(z)^{k}:=\mathbb{C}[s] \bigcap \mathfrak{m}_{z}^{k}=\mathbb{C}[s](s-z)^{k} .
\end{gathered}
$$

The canonical injection $\mathbb{C}[s] \subset \mathcal{O}_{z}=\mathbb{C}<s-z>$ induces the isomorphism

$$
\mathbb{C}[s] / \mathbb{C}[s](s-z)^{k} \cong \mathbb{C}<s-z>/ \mathbb{C}<s-z>(s-z)^{k} \cong \oplus_{j=0}^{k-1} \mathbb{C} \overline{(s-z)}^{j}
$$

where $\overline{s-z}$ denotes the residue class in the respective factor algebras. Together the equations (27) and (28) induce the canonical identifications

$$
\begin{gathered}
\mathcal{O}_{z} / \mathcal{O}_{z}(s-z)^{k}=\mathcal{O} / \mathcal{O}(s-z)^{k}=\mathcal{E}_{\mathfrak{m}_{E}(z)}^{\prime} / \mathcal{E}_{\mathfrak{m}_{E}(z)}^{\prime}(s-z)^{k(z)}=\mathcal{E}^{\prime} / \mathcal{E}^{\prime}(s-z)^{k}= \\
\mathbb{C}[s] / \mathbb{C}[s](s-z)^{k}=\oplus_{i=0}^{k-1} \mathbb{C} \overline{(s-z)} \cong \mathbb{C}[s-z]_{<k}=\mathbb{C}[s]_{<k} .
\end{gathered}
$$

These factor algebras have $\mathbb{C}$-dimension $k$. By (22) $\mathcal{E}^{\prime}(s-z)^{k}$ is closed and hence the algebra $\mathcal{E}^{\prime} / \mathcal{E}^{\prime}(s-z)^{k}$ is Hausdorff with the coinduced factor topology and this coincides with the topology as f.d. $\mathbb{C}$-space. The identifications (29) finally induce the identification of the completions of these DRVs [24, §8, p.62-63], [5, (94)], viz.

$$
\widehat{\mathcal{O}_{z}}=\widehat{\mathcal{O}_{\mathfrak{m}_{O}(z)}}=\widehat{\mathcal{E}_{\mathfrak{m}_{E}(z)}^{\prime}}=\widehat{\mathbb{C}[s]_{\mathfrak{m}_{P}(z)}}=\mathbb{C}[[s-z]]
$$

where $\mathbb{C}[[s-z]]$ is the DVR of formal power series in $s-z$. Since the inclusion $A \subset \widehat{A}$ of a local noetherian ring into its completion is faithfully flat, i.e., the functor $M \mapsto \widehat{A} \otimes_{A} M$ preserves and reflects exact sequences of $A$-modules, the preceding considerations imply that all inclusions of DVRs

$$
\mathbb{C}[[s-z]] \supset \mathcal{O}_{z} \supset \mathcal{O}_{\mathfrak{m}_{O}(z)} \supset \mathcal{E}_{\mathfrak{m}_{E}(z)}^{\prime} \supset \mathbb{C}[s]_{\mathfrak{m}_{P}(z)},
$$

are faithfully flat.

Let $\mathfrak{b}=\mathcal{O} f$ be any nonzero closed ideal of $\mathcal{O}$. Its associated analytic variety is the the countable discrete set of zeros of $f$ or $\mathfrak{b}$, viz.

$$
V_{\mathbb{C}}(f):=V_{\mathbb{C}}(\mathfrak{b}):=\{z \in \mathbb{C} ; \forall g \in \mathfrak{b}: g(z)=0\} .
$$

Recall that $\mathfrak{m}_{O}(z)^{k}=\mathcal{O}(s-z)^{k} \subset \mathfrak{m}_{z}^{k}=\mathcal{O}_{z}(s-z)^{k}$. The multiplicity $k(z):=$ $\operatorname{mult}(f, z)$ of $f$ at $z$ is defined by the equivalent conditions

$$
\begin{gathered}
f \in \mathfrak{m}_{O}(z)^{k(z)} \backslash \mathfrak{m}_{O}(z)^{k(z)+1} \Longleftrightarrow f \in \mathfrak{m}_{z}^{k(z)} \backslash \mathfrak{m}_{z}^{k(z)+1} \\
\Longleftrightarrow \exists g \in \mathcal{O} \text { with } f=g(s-z)^{k(z)} \text { and } g(z) \neq 0 \Longleftrightarrow \\
f^{(i)}(z)=0 \text { for } i=0, \cdots, k(z)-1, f^{(k(z))}(z) \neq 0,
\end{gathered}
$$


hence $V_{\mathbb{C}}(f)=\{z \in \mathbb{C} ; \operatorname{mult}(f, z) \geq 1\}$.

Definition 3.2. For any nonzero ideal $\mathfrak{b}=\mathcal{O} f, 0 \neq f \in \mathcal{O}$, the family

$$
\left(V_{\mathbb{C}}(f),(\operatorname{mult}(f, z))_{z \in V_{\mathbb{C}}(f)}\right)
$$

is called the cospectrum [30, p. 911] or multiplicity variety [1, p. 117] of $\mathfrak{b}$. If $\mathcal{B} \subsetneq \mathcal{E}$ is a gen.beh., i.e., a proper closed translation-invariant subspace of $\mathcal{E}$, with its associated nonzero ideals $\mathfrak{a}:=\mathcal{B}^{\perp}$ of $\mathcal{E}^{\prime}$ and $\mathfrak{b}:=\operatorname{cl}_{O}(\mathcal{O} \mathfrak{a})$ of $\mathcal{O}$ then the cospectrum of $\mathfrak{b}$ is called the spectrum of $\mathcal{B}[30$, p. 877].

The Weierstraß and Mittag-Leffler theorems (compare also [14, Thm. 7]) imply the Stein algebra isomorphism

$$
\begin{gathered}
\Delta: \mathcal{O} / \mathcal{O} f \cong \prod_{z \in V_{\mathbb{C}}(f)} \mathcal{O} / \mathcal{O}(s-z)^{k(z)}, k(z):=\operatorname{mult}(f, z), \\
g+\mathcal{O} f \mapsto\left(g+\mathcal{O}(s-z)^{k(z)}\right)_{z \in V_{\mathbb{C}}(f)} \\
\text { with } \operatorname{ker}(\Delta)=\mathfrak{b}=\bigcap_{z \in V_{\mathbb{C}}(f)} \mathcal{O}(s-z)^{k(z)} \text { and } \\
\mathcal{O} / \mathcal{O}(s-z)^{k(z)} \underset{(29)}{=} \mathbb{C}[s] / \mathbb{C}[s](s-z)^{k(z)}= \\
\oplus_{j=0}^{k(z)-1} \mathbb{C} \overline{(s-z)^{j}} \ni \bar{g}:=g+\mathcal{O} f=\sum_{i=0}^{k(z)-1} \frac{g^{(j)}(z)}{j !} \overline{(s-z)^{j}}
\end{gathered}
$$

This implies in particular that the cospectra or multiplicity varieties are in one-one correspondence with the nonzero closed ideals of $\mathcal{O}$. Notice that for finite $V_{\mathbb{C}}(f)$ the isomorphism $\Delta$ is a consequence of the Chinese Remainder Theorem. There is no simple characterization of the closed ideals $\mathfrak{b}$ of $\mathcal{O}$ or of the corresponding multiplicity varieties for which there is a distribution $f \in \mathcal{E}^{\prime}$ with $\mathfrak{b}=\mathcal{O} f$.

\section{Injectivity, elimination and closure}

Elimination in Willems' sense for gen. beh. occurs as follows: Assume

$$
\begin{gathered}
P \in \mathcal{E}^{\prime \ell_{2} \times \ell_{1}}, U_{1} \subseteq \mathcal{E}^{\prime 1 \times \ell_{1}} \text { and } \\
U_{2}:=(\circ P)^{-1}\left(U_{1}\right)=\left\{\eta \in \mathcal{E}^{\prime 1 \times \ell_{2}} ; \eta P \in U_{1}\right\} .
\end{gathered}
$$

The $U_{i}$ induce f.g. modules $M_{i}:=\mathcal{E}^{1 \times \ell_{i}} / U_{i}$, gen. beh.

$$
\begin{gathered}
\mathcal{B}_{i}:=U_{i}^{\perp}=\operatorname{cl}_{E}\left(U_{i}\right)^{\perp} \underset{\text { ident. }}{=} \operatorname{Hom}_{\mathcal{E}^{\prime}}\left(M_{i}, \mathcal{E}\right) \subseteq \mathcal{E}^{\ell_{i}} \\
\text { and maps }(\circ P)_{\text {ind }}: \mathcal{E}^{\prime 1 \times \ell_{2}} / U_{2} \rightarrow \mathcal{E}^{\prime 1 \times \ell_{1}} / U_{1}, \eta+U_{2} \mapsto \eta P+U_{1}, \\
P \circ=\operatorname{Hom}\left((\circ P)_{\text {ind }}, \mathcal{E}\right): \mathcal{B}_{1}=\operatorname{Hom}_{\mathcal{E}^{\prime}}\left(M_{1}, \mathcal{E}\right) \rightarrow \mathcal{B}_{2} .
\end{gathered}
$$

The map $(\circ P)_{\text {ind }}$ is, of course, injective.

Lemma 4.1. For the not necessarily closed submodule $U_{1} \subseteq \mathcal{E}^{\prime 1 \times \ell_{1}}$ the equation

$$
\operatorname{cl}_{E}\left(P \circ U_{1}^{\perp}\right)=\left((\circ P)^{-1}\left(\operatorname{cl}_{E}\left(U_{1}\right)\right)\right)^{\perp}
$$

holds. 
Proof. Let $\xi \in \mathcal{E}^{\prime 1 \times \ell_{2}}$ be any element. We prove the dual equation

$$
\begin{gathered}
\left(P \circ U_{1}^{\perp}\right)^{\perp}=\left(\operatorname{cl}_{E}\left(P \circ U_{1}^{\perp}\right)\right)^{\perp}=\left((\circ P)^{-1}\left(\mathrm{cl}_{E}\left(U_{1}\right)\right)\right)^{\perp \perp}=(\circ P)^{-1}\left(\operatorname{cl}_{E}\left(U_{1}\right)\right) . \\
\text { But } \xi \in\left(P \circ U_{1}^{\perp}\right)^{\perp} \Longleftrightarrow \xi \circ\left(P \circ U_{1}^{\perp}\right)=(\xi P) \circ U_{1}^{\perp}=0 \Longleftrightarrow \\
\xi P \in U_{1}^{\perp \perp}=\operatorname{cl}_{E}\left(U_{1}\right) \Longleftrightarrow \xi \in(\circ P)^{-1}\left(\operatorname{cl}_{E}\left(U_{1}\right)\right) .
\end{gathered}
$$

In Thm. 4.4,(2), below we derive the sharper equation

$$
\operatorname{cl}_{E}\left(P \circ \mathcal{B}_{1}\right)=\operatorname{cl}_{E}\left(P \circ U_{1}^{\perp}\right)=(\circ P)^{-1}\left(U_{1}\right)^{\perp}=\mathcal{B}_{2}
$$

for any, not necessarily closed $U_{1}$.

With the notations from (36) $P \circ \mathcal{B}_{1}$ is, of course, an $\mathcal{E}^{\prime}$-submodule of $\mathcal{E}^{\prime 1 \times \ell_{2}}$. If it is also closed and thus the gen. beh. $\mathcal{B}_{2}$ by (38) then we say by slightly generalizing Willems' terminology in the case of differential systems that $\mathcal{B}_{2}$ is obtained by elimination from $\mathcal{B}_{1}$. Even if $U_{1}$ is f.g. it is not known in general whether $U_{2}$ is also f.g.. In other words, the closed image of a behavior may not be a behavior and therefore the treatment of gen. beh. instead of behaviors only is mandatory. If all gen. beh. are behaviors then this problem disappears, but this is not known at present.

A module $\mathcal{E}^{\prime} W$ is injective if and only if the left exact functor $\operatorname{Hom}_{\mathcal{E}^{\prime}}(-, W)$ is even exact or, equivalently, transforms injections into surjections, and an injective cogenerator if in addition this functor also reflects exactness or if $\operatorname{Hom}_{\mathcal{E}^{\prime}}(M, W)=0 \mathrm{im}$ plies $M=0$. If $0 \neq f \in \mathcal{E}^{\prime}=\mathcal{E}^{\prime 1 \times 1}$ is not invertible the map $\circ f: \mathcal{E}^{\prime} \rightarrow \mathcal{E}^{\prime}$ is injective, but $f \circ=\operatorname{Hom}(\circ f, \mathcal{E}): \mathcal{E} \rightarrow \mathcal{E}$ is not surjective and therefore $f \circ \mathcal{E}$ is not a gen. beh. and $\mathcal{E}^{\prime} \mathcal{E}$ is not injective and does not admit elimination. But we are now going to show that $\mathcal{P} \mathcal{E}$ admits elimination. By [26, Thms. 1.14, 6.6] the module $\mathcal{P} \mathcal{E}(-i z)=\mathbb{C}[t] e^{-i z t}$ is the minimal injective cogenerator over the local ring $\mathbb{C}[s]_{\mathfrak{m}_{P}(z)}$ where $s=i \partial, \mathbb{C}[s]=\mathbb{C}[\partial]$ and $\mathfrak{m}_{P}(z)=\mathbb{C}[s](s-z)=\mathbb{C}[\partial](\partial+i z)$. According to $[24$, Thm. 18.6,(iii)] the module $\mathcal{P} \mathcal{E}(-i z)$ is also the minimal injective cogenerator over the completion $\widehat{\mathbb{C}[s]_{\mathfrak{m}_{P}(z)}}=\mathbb{C}[[s-z]]=\mathbb{C}[[\partial+i z]]$. Here the action of $\mathbb{C}[s]=\mathbb{C}[\partial]$ on $\mathcal{P} \mathcal{E}(-i z)$ by differentiation can be canonically extended to $\mathbb{C}[[s-z]]$ since $(\partial+i z)^{m} \circ \frac{t^{j}}{j !} e^{-i z t}=0$ for $m>j$ by (14). This latter nilpotence of the action moreover implies that $\mathbb{C}[s]$ - and $\mathbb{C}[[s-z]]$-submodules of $\mathcal{P} \mathcal{E}(-i z)$ coincide. The faithful flatness of the inclusions (31) then furnish the injectivity of $\mathcal{P} \mathcal{E}(-i z)$ as $\mathcal{E}_{\mathfrak{m}_{E}(z)}^{\prime}$-module. We give a simple, essentially constructive direct proof of this result.

Consider the formal power series algebra $\mathbb{C}[[\partial]]$ and the polynomial ring $\mathbb{C}[t]=$ $\oplus_{j=0}^{\infty} \mathbb{C} t^{j}$ with its basis $e_{j}:=t^{j} / j !, j \in \mathbb{N}$, and the $\mathbb{C}$-linear differentiation operator

$$
\partial=\frac{d}{d t}: \mathbb{C}[t] \rightarrow \mathbb{C}[t], w \mapsto w^{\prime}=\frac{d w}{d t}=\partial w, \partial^{m} e_{j}=\left\{\begin{array}{ll}
e_{j-m} & \text { if } m \leq j \\
0 & \text { if } m>j
\end{array} .\right.
$$

This endomorphism can be extended to the action

$$
\begin{gathered}
\circ: \mathbb{C}[[\partial]] \times \mathbb{C}[t] \rightarrow \mathbb{C}[t], \sum_{m=0}^{\infty} a_{m} \partial^{m} \circ w:=\sum_{m \leq \operatorname{deg}(w)} a_{m} \partial^{m} w, \text { or } \\
\sum_{m=0}^{\infty} a_{m} \partial^{m} \circ \sum_{j=0}^{k} b_{j} e_{j}:=\sum_{m \leq j \leq k} a_{m} b_{j} e_{j-m} .
\end{gathered}
$$


which makes $\mathbb{C}[t]$ a module over the DVR $\mathbb{C}[[\partial]]$. Since $\mathbb{C}[[\partial]]$ is a principal ideal domain the injectivity of $\mathbb{C}[[\partial]] \mathbb{C}[t]$ is equivalent to its divisibility, i.e., to the solvability of $f \circ y=v$ for each $v \in \mathbb{C}[t]$ and nonzero $f \in \mathbb{C}[[\partial]]$. It suffices to show this solvability for the basis signals $v:=e_{k}=t^{k} / k !$. If $f=g \partial^{m}$ with a unit $g$ of $\mathbb{C}[[\partial]]$ and $y:=g^{-1} \circ e_{k+m}$ then $f \circ y=v=e_{k}$, indeed

$$
\begin{gathered}
f=g \partial^{m}, g=g_{0}+g_{1} \partial+\cdots, g_{0} \neq 0, g^{-1}=\sum_{n=0}^{\infty} a_{n} \partial^{n}, a_{0}=g_{0}^{-1}, \\
y:=g^{-1} \circ e_{k+m}=\sum_{n=0}^{k+m} a_{n} e_{k+m-n} . \text { Then } \\
f \circ y=\partial^{m} \circ g \circ g^{-1} \circ e_{k+m}=\partial^{m} \circ e_{k+m}=e_{k+m-m}=e_{k}=v .
\end{gathered}
$$

The maximal ideal of $\mathbb{C}[[\partial]]$ is $\mathfrak{m}:=\mathbb{C}[[\partial]] \partial$ and the map

$$
\mathbb{C}[[\partial]] \rightarrow \mathbb{C}[t], f=f_{0}+f_{1} \partial+\cdots \mapsto f \circ 1=f_{0}=f_{0} t^{0},
$$

induces the $\mathbb{C}[[\partial]]$-isomorphism $\mathbb{C}[[\partial]] / \mathfrak{m} \cong \mathbb{C}=\mathbb{C} t^{0}$, hence $\mathbb{C} t^{0}$ is the unique simple $\mathbb{C}[[\partial]]$-module (up to isomorphism).

Lemma 4.2. (cf. [24, Thm. Ex. 18.7]) The module $\mathbb{C}[t]$ is the unique least injective $\mathbb{C}[[\partial]]$-cogenerator (up to isomorphism).

Proof. This is a standard result of homological algebra of which we give a direct proof. (i) Let $N$ be a nonzero $\mathbb{C}[[\partial]]$-module. Since the ring is noetherian there is a simple subquotient $U_{1} / U_{2}, U_{2} \subset U_{1} \subseteq N$, hence $U_{1} / U_{2} \cong \mathbb{C} t^{0}$. Since $\mathbb{C}[t]$ is injective the nonzero linear map $U_{1} \stackrel{\text { can }}{\longrightarrow} U_{1} / U_{2} \cong \mathbb{C} t^{0} \subset \mathbb{C}[t]$ can be extended to $N$ and therefore $\operatorname{Hom}_{\mathbb{C}[\partial]]]}(N, \mathbb{C}[t]) \neq 0$. This shows that $\mathbb{C}[[\partial]] \mathbb{C}[t]$ is an injective cogenerator.

(ii) If $V \subseteq \mathbb{C}[t]$ is a nonzero submodule and $0 \neq w=\sum_{k=0}^{m} a_{k} e_{k} \in V, a_{m} \neq 0$, then

$$
0 \neq a_{m}=a_{m} t^{0}=\partial^{m} \circ w \in V \bigcap \mathbb{C} t^{0} \Longrightarrow V \bigcap \mathbb{C} t^{0} \neq 0 .
$$

This signifies [24, p.281] that $\mathbb{C} t^{0}$ is essential in $\mathbb{C}[t]$.

(iii) If $W$ is any injective cogenerator then $\operatorname{Hom}_{\mathbb{C}[\partial]]}\left(\mathbb{C} t^{0}, W\right) \neq 0$. Since $\mathbb{C} t^{0}$ is simple and $W$ is injective a nonzero $\mathbb{C}[[\partial]]$-linear map $\varphi: \mathbb{C} t^{0} \rightarrow W$ is obviously injective and can be extended to $\psi: \mathbb{C}[t] \rightarrow W$ with

$$
\begin{gathered}
0=\operatorname{ker}(\varphi)=\mathbb{C} t^{0} \bigcap \operatorname{ker}(\psi) \underset{\left({ }_{(i i)}\right.}{\Longrightarrow} \operatorname{ker}(\psi)=0 \underset{\text { ident. }}{\Longrightarrow} \\
\mathbb{C}[t] \subset W \underset{\mathbb{C}[t] \text { injective }}{\Longrightarrow} \mathbb{C}[t] \bigoplus W_{1}=W .
\end{gathered}
$$

This shows that $\mathbb{C}[t]$ is indeed the least injective $\mathbb{C}[[\partial]]$-cogenerator (up to isomorphism).

We now transfer this result to other situations. Define the isomorphisms

$$
\begin{gathered}
\phi: \mathbb{C}[t] \cong \mathcal{P} \mathcal{E}(-i z)=\mathbb{C}[t] e^{-i z t} \\
w \mapsto w e^{-i z t}, e_{j}=t^{j} / j ! \mapsto e_{-i z, j}=t^{j}(j !)^{-1} e^{-i z t} \text { and } \\
\varphi: \mathbb{C}[[\partial]] \cong \mathbb{C}[[s-z]], \\
\partial \mapsto \partial+i z=i^{-1}(s-z), \sum_{m} a_{m} \partial^{m} \mapsto \sum_{m} a_{m} i^{-m}(s-z)^{m} .
\end{gathered}
$$


The map $\varphi$ is an algebra isomorphism and $\phi$ is $\varphi$-semi-linear in the sense that

$$
\begin{gathered}
\phi(f \circ w)=\varphi(f) \circ \phi(w), f \in \mathbb{C}[[\partial]], w \in \mathbb{C}[t], \text { especially for } m \leq j: \\
\phi\left(\partial^{m} \circ e_{j}\right)=\phi\left(e_{j-m}\right)=e_{-i z, j-m}=(\partial+i z)^{m} \circ e_{-i z, j}=\varphi\left(\partial^{m}\right) \circ \phi\left(e_{j}\right) .
\end{gathered}
$$

Lemma 4.2 and a standard transport of structure argument furnish

Corollary 4.3. For $z \in \mathbb{C}$ the modules $\mathcal{P E}(-i z)=\mathbb{C}[t] e^{-i z t}$ resp. $\mathcal{P E}(z)=\mathbb{C}[t] e^{z t}$ are the unique least injective cogenerators over the formal power series algebras $\mathbb{C}[[s-z]]=\mathbb{C}[[\partial+i z]]$ resp. $\mathbb{C}[[\partial-z]]=\mathbb{C}[[s-i z]]$. If

$$
\begin{gathered}
f=g(s-z)^{m}, g \in \mathbb{C}[[s-z]], g(z) \neq 0, g^{-1}=\sum_{n=0}^{\infty} a_{n}(s-z)^{n}, u:=e_{-i z, k} \text { and } \\
y:=g^{-1} \circ i^{-m} e_{-i z, k+m}=\sum_{n=0}^{k+m} a_{n} i^{n-m} e_{-i z, k+m-n} \text { then } f \circ y=u .
\end{gathered}
$$

The last equation yields the constructive solution $y$ of the divisibility equation $f \circ y=u$.

Proof. The proof is a modification of those of (41) and of Lemma 4.2.

We now transfer this result to the local rings $\mathcal{E}_{\mathfrak{m}_{E}(z)}^{\prime}$ and also to $\mathcal{E}^{\prime}$.

Theorem 4.4. Let $\mathcal{B} \subseteq \mathcal{E}^{\ell}$ be any gen. beh..

1. The module $\mathcal{P} \mathcal{E}(-i z)=\mathbb{C}[t] e^{-i z t}$ is the least injective cogenerator over $\mathcal{E}_{\mathfrak{m}_{E}(z)}^{\prime}$. In particular, there is a matrix $R^{z} \in \mathcal{E}^{\prime k_{z} \times \ell}$ that is of maximal row rank $k_{z}$ and unique up to row equivalence over $\mathcal{E}_{\mathfrak{m}_{E}(z)}^{\prime}$ such that

$$
\mathcal{B} \bigcap \mathcal{P E}(-i z)^{\ell}=\left\{w \in \mathcal{P E}(z)^{\ell} ; R^{z} \circ w=0\right\} .
$$

2. The module $\mathcal{P} \mathcal{E}=\bigoplus_{z \in \mathbb{C}} \mathbb{C}[t] e^{z t}=\bigoplus_{z \in \mathbb{C}} \mathbb{C}[t] e^{-i z t}$ is injective for f.g. $\mathcal{E}^{\prime}$ modules, i.e., the left exact functor $\operatorname{Hom}_{\mathcal{E}^{\prime}}(-, \mathcal{P} \mathcal{E})$ preserves exactness of sequences off.g. modules and especially transforms injections $M^{\prime} \rightarrow M$ off.g. $\mathcal{E}^{\prime}$-modules into surjections $\operatorname{Hom}_{\mathcal{E}^{\prime}}(M, \mathcal{P} \mathcal{E}) \rightarrow \operatorname{Hom}_{\mathcal{E}^{\prime}}\left(M^{\prime}, \mathcal{P} \mathcal{E}\right)$. For the data from 1. resp. (36) this implies

$$
\begin{gathered}
\mathcal{B} \bigcap \mathcal{P} \mathcal{E}^{\ell}=\bigoplus_{z \in \mathbb{C}}\left\{w \in \mathcal{P E}(-i z)^{\ell} ; R^{z} \circ w=0\right\} \text { resp. } \\
P \circ\left(\mathcal{B}_{1} \bigcap \mathcal{P} \mathcal{E}^{\ell}\right)=\mathcal{B}_{2} \bigcap \mathcal{P} \mathcal{E}^{\ell}, \operatorname{cl}_{E}\left(P \circ \mathcal{B}_{1}\right)=\mathcal{B}_{2} .
\end{gathered}
$$

In particular ${ }_{\mathcal{E}^{\prime}} \mathcal{P} \mathcal{E}$ admits elimination.

Proof. 1. The divisibilty of $\mathcal{P} \mathcal{E}(-i z)$ over $\mathbb{C}[[s-z]]$ implies that over the subring $\mathcal{E}_{\mathfrak{m}_{E}(z)}^{\prime}$. Notice that $\mathbb{C}[s]=\mathbb{C}\left[-i \delta^{\prime}\right] \subset \mathcal{E}_{\mathfrak{m}_{E}(z)}^{\prime} \subset \mathbb{C}[[s-z]]$. If

$$
\begin{gathered}
0 \neq w=\sum_{j=0}^{m} w_{j} e_{-i z, j} \in \mathcal{P} \mathcal{E}(-i z)=\mathbb{C}[t] e^{-i z t}, w_{j} \in \mathbb{C}, w_{m} \neq 0, \text { then } \\
0 \neq(s-z)^{m} \circ w=i^{m} w_{m} e^{-i z t} \in \mathbb{C} e^{-i z t} .
\end{gathered}
$$

As in Lemma 4.2 this shows that the simple $\mathcal{E}_{\mathfrak{m}_{E}(z)}^{\prime}$-module $\mathbb{C} e^{-i z t}$ is essential in $\mathcal{P E}(-i z)$ and that $\mathcal{P} \mathcal{E}(-i z)$ is the least injective $\mathcal{E}_{\mathfrak{m}_{E}(z)}^{\prime}$-cogenerator.

2. For all $z \in \mathbb{C}$ the localization or quotient module functor

$$
\operatorname{Mod}_{\mathcal{E}^{\prime}} \rightarrow \operatorname{Mod}_{\mathcal{E}_{\mathfrak{m}_{E}(z)}^{\prime}}, M \mapsto M_{\mathfrak{m}_{E}(z)}:=\left\{f^{-1} x ; x \in M, f \in \mathcal{E}^{\prime} \backslash \mathfrak{m}_{E}(z)\right\}
$$


is exact and so is the functor

$$
\begin{aligned}
& \operatorname{Mod}_{\mathcal{E}^{\prime}} \rightarrow \operatorname{Mod}_{\mathcal{E}_{\mathfrak{m}_{E}(z)}^{\prime}} \\
& M \mapsto \operatorname{Hom}_{\mathcal{E}^{\prime}}(M, \mathcal{P E}(-i z)) \underset{\text { ident. }}{=} \operatorname{Hom}_{\mathcal{E}_{\mathfrak{m}_{E}(z)}^{\prime}}\left(M_{\mathfrak{m}_{E}(z)}, \mathcal{P E}(-i z)\right)
\end{aligned}
$$

since $\mathcal{P E}(-i z)$ is injective as $\mathcal{E}_{\mathfrak{m}_{E}(z)}^{\prime}$-module. The identification comes from the universal property of the quotient module $M_{\mathfrak{m}_{E}(z)}$. If $M$ is a f.g. $\mathcal{E}^{\prime}$-module the functor $\operatorname{Hom}_{\mathcal{E}^{\prime}}(M,-)$ preserves direct sums and hence

$$
\begin{gathered}
\operatorname{Hom}_{\mathcal{E}^{\prime}}(M, \mathcal{P} \mathcal{E})=\operatorname{Hom}_{\mathcal{E}^{\prime}}\left(M, \bigoplus_{z \in \mathbb{C}} \mathcal{P E}(-i z)\right) \underset{\text { ident. }}{=} \bigoplus_{z \in \mathbb{C}} \operatorname{Hom}_{\mathcal{E}^{\prime}}(M, \mathcal{P} \mathcal{E}(-i z)) \underset{\text { ident. }}{=} \\
\bigoplus_{z \in \mathbb{C}} \operatorname{Hom}_{\mathcal{E}_{\mathfrak{m}_{E}(z)}^{\prime}}\left(M_{\mathfrak{m}_{E}(z)}, \mathcal{P E}(-i z)\right) .
\end{gathered}
$$

Since direct sums of exact sequences are exact too this shows that the contravariant functor $M \mapsto \operatorname{Hom}_{\mathcal{E}^{\prime}}(M, \mathcal{P E})$ maps exact sequences $M^{\prime} \rightarrow M \rightarrow M^{\prime \prime}$ of f.g. $\mathcal{E}^{\prime}-$ modules onto exact sequences. In the situation of (35) and (36) this implies the surjection

$$
\begin{aligned}
& P \circ: \mathcal{B}_{1} \bigcap \mathcal{P E}^{\ell_{1}} \underset{\text { ident. }}{=} \operatorname{Hom}_{\mathcal{E}^{\prime}}\left(M_{1}, \mathcal{P E}\right) \rightarrow \mathcal{B}_{2} \bigcap \mathcal{P} \mathcal{E}^{\ell_{2}} \underset{\text { ident. }}{=} \operatorname{Hom}_{\mathcal{E}^{\prime}}\left(M_{2}, \mathcal{P E}\right) \Longrightarrow \\
& \mathcal{B}_{2} \bigcap \mathcal{P} \mathcal{E}^{\ell_{2}}=P \circ\left(\mathcal{B}_{1} \bigcap \mathcal{P} \mathcal{E}^{\ell_{1}}\right) \subseteq P \circ \mathcal{B}_{1} \subseteq \mathcal{B}_{2} \Longrightarrow \\
& \mathcal{B}_{2}=\operatorname{cl}_{E}\left(\mathcal{B}_{2} \bigcap \mathcal{P} \mathcal{E}^{\ell_{2}}\right)=\operatorname{cl}_{E}\left(P \circ \mathcal{B}_{1}\right)
\end{aligned}
$$

With $M=\mathcal{E}^{\prime 1 \times \ell} / U$ and the identifications

$$
\mathcal{B} \underset{\text { ident. }}{=} \operatorname{Hom}_{\mathcal{E}^{\prime}}(M, \mathcal{E}) \text { and } \mathcal{B} \bigcap \mathcal{P E}^{\ell} \underset{\text { ident. }}{=} \operatorname{Hom}_{\mathcal{E}^{\prime}}(M, \mathcal{P E})
$$

equation (49) also implies (45).

Notice that for arbitrarily chosen matrices $R^{z} \in \mathcal{E}^{\prime k_{z} \times \ell}$ the gen. beh. $\mathcal{B}:=$ $\mathrm{cl}_{E}\left(\bigoplus_{z \in \mathbb{C}}\left\{w \in \mathcal{P} \mathcal{E}(z)^{\ell} ; R^{z} \circ w=0\right\}\right)$ does not satisfy (45). This is in contrast to Thm. 5.6 below.

Thm. 4.4 implies the following important result on the connection between algebra and topology. Let

$$
\begin{gathered}
\mathcal{K}:=\operatorname{quot}\left(\mathcal{E}^{\prime}\right):=\left\{f g^{-1} ; f, g \in \mathcal{E}^{\prime}, g \neq 0\right\} \subset \\
\mathcal{M}:=\operatorname{quot}(\mathcal{O})=\left\{f g^{-1} ; f, g \in \mathcal{O}, g \neq 0\right\}
\end{gathered}
$$

denote the quotient field $\mathcal{K}$ of $\mathcal{E}^{\prime}$ inside the quotient field $\mathcal{M}$ of $\mathcal{O}$ of meromorphic functions. All local rings $\mathcal{E}_{\mathfrak{m}_{E}(z)}^{\prime}$ are contained in $\mathcal{K}$. For every submodule $U \subseteq \mathcal{E}^{\prime 1 \times \ell}$ this implies

$$
\begin{gathered}
U_{\mathfrak{m}_{E}(z)} \subseteq \mathcal{E}_{\mathfrak{m}_{E}(z)}^{\prime \times \ell} \subset \mathcal{K}^{1 \times \ell} \text { and } \\
\mathcal{K} U=\mathcal{K} U_{\mathfrak{m}_{E}(z)}=\mathcal{K} \otimes_{\mathcal{E}^{\prime}} U \subseteq \mathcal{K}^{1 \times \ell}=\mathcal{K} \otimes_{\mathcal{E}^{\prime}} \mathcal{E}^{\prime 1 \times \ell} .
\end{gathered}
$$

Theorem 4.5. Let $U \subseteq \mathcal{E}^{\prime 1 \times \ell}$ be any $\mathcal{E}^{\prime}$-submodule and $M:=\mathcal{E}^{\prime 1 \times \ell} / U$.

1. The closure of $U$ in $\mathcal{E}^{1 \times \ell}$ is

$$
\operatorname{cl}_{E}(U)=\mathcal{E}^{\prime 1 \times \ell} \bigcap \bigcap_{z \in \mathbb{C}} U_{\mathfrak{m}_{E}(z)} .
$$


2. The closures in $\mathcal{E}^{1 \times \ell}$ and in $\mathcal{O}^{1 \times \ell}$ are related by

$$
\begin{gathered}
\operatorname{cl}_{O}(U)=\operatorname{cl}_{O}(\mathcal{O U}) \text { and } \operatorname{cl}_{E}(U)=\mathcal{E}^{1 \times \ell} \bigcap \operatorname{cl}_{O}(U), \\
\text { hence } \mathcal{E}^{\prime 1 \times \ell} / \operatorname{cl}_{E}(U) \underset{\text { ident. }}{\subset} \mathcal{O}^{1 \times \ell} / \operatorname{cl}_{O}(U) .
\end{gathered}
$$

Proof. 1. Let

$$
\begin{gathered}
\mathcal{B}=U^{\perp} \underset{\text { ident. }}{=} \operatorname{Hom}_{\mathcal{E}^{\prime}}(M, \mathcal{E}) \subseteq \mathcal{E}^{\ell} \Longrightarrow \operatorname{cl}_{E}(U)=U^{\perp \perp}=\mathcal{B}^{\perp} \underset{\text { Res. } 2.1}{=} \\
\left(\mathcal{B} \bigcap \mathcal{P} \mathcal{E}^{\ell}\right)^{\perp} \underset{(45)}{=}\left(\sum_{z \in \mathbb{C}}\left(\mathcal{B} \bigcap \mathcal{P E}(-i z)^{\ell}\right)\right)^{\perp}=\bigcap_{z \in \mathbb{C}}\left(\mathcal{B} \bigcap \mathcal{P E}(-i z)^{\ell}\right)^{\perp} .
\end{gathered}
$$

Since $\mathcal{P} \mathcal{E}(-i z)$ is an injective cogenerator over $\mathcal{E}_{\mathfrak{m}_{E}(z)}^{\prime}$ the equation

$$
\begin{gathered}
\mathcal{B} \bigcap \mathcal{P E}(-i z)^{\ell} \underset{\text { ident. }}{=} \operatorname{Hom}_{\mathcal{E}_{\mathfrak{m}_{E}(z)}^{\prime}}\left(M_{\mathfrak{m}_{E}(z)}, \mathcal{P E}(-i z)\right)= \\
\operatorname{Hom}_{\mathcal{E}_{\mathfrak{m}_{E}(z)}^{\prime}\left(\mathcal{E}_{\mathfrak{m}_{E}(z)}^{\prime 1 \times \ell} / U_{\mathfrak{m}_{E}(z)}, \mathcal{P E}(-i z)\right)}
\end{gathered}
$$

implies

$$
\begin{gathered}
U_{\mathfrak{m}_{E}(z)}=\left\{\xi \in \mathcal{E}_{\mathfrak{m}_{E}(z)}^{\prime 1 \times \ell} ; \xi \circ\left(\mathcal{B} \bigcap \mathcal{P E}(-i z)^{\ell}\right)=0\right\} \Longrightarrow \\
\mathcal{E}^{\prime 1 \times \ell} \bigcap U_{\mathfrak{m}_{E}(z)}=\left\{\xi \in \mathcal{E}^{\prime 1 \times \ell} ; \xi \circ\left(\mathcal{B} \bigcap \mathcal{P E}(-i z)^{\ell}\right)=0\right\}=\left(\mathcal{B} \bigcap \mathcal{P E}(-i z)^{\ell}\right)^{\perp} \\
\Longrightarrow \mathcal{E}^{\prime \times \ell} \bigcap \bigcap_{z \in \mathbb{C}} U_{\mathfrak{m}_{E}(z)}=\bigcap_{z \in \mathbb{C}}\left(\mathcal{B} \bigcap \mathcal{P E}(-i z)^{\ell}\right)^{\perp}=\mathcal{B}^{\perp}=\operatorname{cl}_{E}(U) .
\end{gathered}
$$

2. Since $\mathbb{C}[s]$ is contained in $\mathcal{E}^{\prime}$ and dense in $\mathcal{O}$ and since $\mathcal{O} \rightarrow \mathcal{O}^{1 \times \ell}, f \mapsto f x$, is continuous for all $x \in \mathcal{O}^{1 \times \ell}$ we infer

$\forall u \in U: \mathcal{O} u=\operatorname{cl}_{O}(\mathbb{C}[s]) u \subseteq \operatorname{cl}_{O}(\mathbb{C}[s] u) \subseteq \operatorname{cl}_{O}(U) \Longrightarrow \operatorname{cl}_{O}(\mathcal{O} U)=\operatorname{cl}_{O}(U)$

We use the commutative diagrams

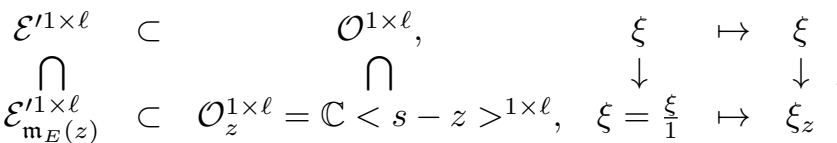

$$
\begin{aligned}
& \text { hence } \mathcal{O}_{z} \mathcal{E}_{\mathfrak{m}_{E}(z)}^{\prime} U=\mathcal{O}_{z} U_{\mathfrak{m}_{E}(z)}=\mathcal{O}_{z} \mathcal{O} U \text {. }
\end{aligned}
$$

Equation (23) implies

$$
\begin{gathered}
\operatorname{cl}_{O}(\mathcal{O} U)=\left\{\eta \in \mathcal{O}^{1 \times \ell} ; \forall z \in \mathbb{C}: \eta_{z} \in \mathcal{O}_{z} \mathcal{O} U=\mathcal{O}_{z} U_{\mathfrak{m}_{E}(z)}\right\} \Longrightarrow \\
\mathcal{E}^{\prime 1 \times \ell} \bigcap \operatorname{cl}_{O}(\mathcal{O} U)=\left\{\xi \in \mathcal{E}^{\prime 1 \times \ell} ; \forall z \in \mathbb{C}: \xi_{z} \in \mathcal{O}_{z} U_{\mathfrak{m}_{E}(z)}\right\}= \\
\left\{\xi \in \mathcal{E}^{\prime 1 \times \ell} ; \forall z \in \mathbb{C}: \xi \in \mathcal{E}_{\mathfrak{m}_{E}(z)}^{\prime \times \ell} \bigcap \mathcal{O}_{z} U_{\mathfrak{m}_{E}(z)}\right\} .
\end{gathered}
$$

Since the inclusion $\mathcal{E}_{\mathfrak{m}_{E}(z)}^{\prime} \subset \mathcal{O}_{z}$ is faithfully flat by (31) the following equality holds for every submodule $V \subseteq \mathcal{E}_{\mathfrak{m}_{E}(z)}^{1 \times \ell}$ :

$$
\begin{gathered}
V=\mathcal{E}_{\mathfrak{m}_{E}(z)}^{\prime} \bigcap \mathcal{O}_{z} V \Longrightarrow U_{\mathfrak{m}_{E}(z)}=\mathcal{E}_{\mathfrak{m}_{E}(z)}^{\prime 1 \times \ell} \bigcap \mathcal{O}_{z} U_{\mathfrak{m}_{E}(z)} \Longrightarrow \mathcal{E}^{\prime 1 \times \ell} \bigcap \mathrm{cl}_{O}(\mathcal{O} U)= \\
\left\{\xi \in \mathcal{E}^{\prime 1 \times \ell} ; \forall z \in \mathbb{C}: \xi \in U_{\mathfrak{m}_{E}(z)}\right\}=\mathcal{E}^{\prime 1 \times \ell} \bigcap \bigcap_{z \in \mathbb{C}} U_{\mathfrak{m}_{E}(z)}=\operatorname{cl}_{E}(U) .
\end{gathered}
$$


This theorem enables the study of $\mathcal{E}^{1 \times \ell} / \mathrm{cl}_{E}(U)$ by means of the f.g. modules $\mathcal{E}_{\mathfrak{m}(z)}^{\prime 1 \times \ell} / U_{\mathfrak{m}(z)}$ over the DVRs $\mathcal{E}_{\mathfrak{m}(z)}^{\prime}$ with their well-known simple structure.

Corollary 4.6. ([34, Prop. 8]) If $U=\mathcal{E}^{1 \times k} R, R \in \mathcal{E}^{\prime k \times \ell}$, is f.g. then $\mathcal{O} U=\mathcal{O}^{1 \times k} R$ is f.g. and thus closed and therefore $\mathrm{cl}_{E}\left(\mathcal{E}^{1 \times k} R\right)=\mathcal{E}^{1 \times \ell} \cap \mathcal{O}^{1 \times k} R$. In particular, $\operatorname{cl}_{E}\left(\mathcal{E}^{\prime} f\right)=\mathcal{E}^{\prime} \cap \mathcal{O} f$ for all $f \in \mathcal{E}^{\prime}$ and this implies the first equivalence in (22).

\section{Corollary 4.7. Assume}

$P \in \mathcal{E}^{\prime \ell_{2} \times \ell_{1}}, U_{i} \subseteq \mathcal{E}^{\prime 1 \times \ell_{i}}, \mathcal{B}_{i}:=U_{i}^{\perp} \subseteq \mathcal{E}^{\ell_{i}}, U_{1} P \subseteq U_{2}$, hence $P \circ \mathcal{B}_{1} \subseteq \mathcal{B}_{2}$.

1. If the $U_{i}$ are closed then $\operatorname{cl}_{E}\left(P \circ \mathcal{B}_{1}\right)=\mathcal{B}_{2}$ if and only if $U_{2}=(\circ P)^{-1}\left(U_{1}\right)$.

2. The map $P \circ: \mathcal{B}_{1} \rightarrow \mathcal{B}_{2} \subseteq \mathcal{E}^{\ell_{2}}$ is injective if and only if

$$
\operatorname{cl}_{E}\left(\mathcal{E}^{\prime 1 \times \ell_{2}} P+U_{1}\right)=\mathcal{E}^{\prime 1 \times \ell_{1}}, \text { i.e. }, \forall z \in \mathbb{C}: \mathcal{E}_{\mathfrak{m}_{E}(z)}^{\prime 1 \times \ell_{2}} P+U_{1, \mathfrak{m}_{E}(z)}=\mathcal{E}_{\mathfrak{m}_{E}(z)}^{\prime 1 \times \ell_{2}} .
$$

Proof. 1. $\Longleftarrow:(46) . \Longrightarrow$ : From the assumption and (46) we infer

$$
U_{2}^{\perp}=\mathcal{B}_{2}=\operatorname{cl}_{E}\left(P \circ \mathcal{B}_{1}\right)=(\circ P)^{-1}\left(U_{1}\right)^{\perp} \Longrightarrow U_{2}=(\circ P)^{-1}\left(U_{1}\right)
$$

since $U_{2}, U_{1}$ and thus $(\circ P)^{-1}\left(U_{1}\right)$ are closed.

2. Application of the left exact functor $\operatorname{Hom}_{\mathcal{E}^{\prime}}(-, \mathcal{E})$ to the exact sequence

$$
\mathcal{E}^{\prime 1 \times \ell_{2}} \stackrel{(\circ P)_{\text {ind }}}{\longrightarrow} \mathcal{E}^{\prime 1 \times \ell_{1}} / U_{1} \stackrel{\text { can }}{\longrightarrow} \mathcal{E}^{\prime} / U_{3} \rightarrow 0, U_{3}:=\mathcal{E}^{\prime 1 \times \ell_{2}} P+U_{1},
$$

of $\mathcal{E}^{\prime}$-modules furnishes the exact gen. beh. sequence

$$
\begin{aligned}
& 0 \rightarrow U_{3}^{\perp} \underset{\text { ident. }}{=} \operatorname{Hom}_{\mathcal{E}^{\prime}}\left(\mathcal{E}^{\prime 1 \times \ell_{1}} / U_{3}, \mathcal{E}\right) \rightarrow \mathcal{B}_{1}=U_{1}^{\perp} \stackrel{P \circ}{\longrightarrow} \mathcal{E}^{\ell_{2}} \text {, hence } \\
& P \circ: \mathcal{B}_{1} \rightarrow \mathcal{E}^{\ell_{2}} \text { is injective } \Longleftrightarrow \operatorname{cl}_{E}\left(U_{3}\right)^{\perp}=U_{3}^{\perp}=0 \Longleftrightarrow \operatorname{cl}_{E}\left(U_{3}\right)=\mathcal{E}^{\prime 1 \times \ell_{1}} \\
& \underset{(52)}{\Longleftrightarrow} \forall z \in \mathbb{C}: \mathcal{E}_{\mathfrak{m}_{E}(z)}^{\prime 1 \times \ell_{2}} P+U_{1, \mathfrak{m}_{E}(z)}=U_{3, \mathfrak{m}_{E}(z)}=\mathcal{E}_{\mathfrak{m}_{E}(z)}^{\prime 1 \times \ell_{2}} \text {. }
\end{aligned}
$$

Corollary 4.8. If $\mathcal{O}^{1 \times \ell_{3}} \stackrel{\circ Q}{\longrightarrow} \mathcal{O}^{1 \times \ell_{2}} \stackrel{\circ P}{\longrightarrow} \mathcal{O}^{1 \times \ell_{1}}, Q \in \mathcal{E}^{\prime \ell_{3} \times \ell_{2}}, P \in \mathcal{E}^{\prime \ell_{2} \times \ell_{1}}$, is exact then $\mathrm{cl}_{E}\left(P \circ \mathcal{E}^{\ell_{1}}\right)=\left\{w \in \mathcal{E}^{\ell_{2}} ; Q \circ w=0\right\}$.

Proof. Let $\mathcal{B}_{2}:=\left\{w \in \mathcal{E}^{\ell_{2}} ; Q \circ w=0\right\}$. Then

$$
\begin{aligned}
& \mathcal{O}^{1 \times \ell_{3}} \stackrel{\circ Q}{\longrightarrow} \mathcal{O}^{1 \times \ell_{2}} \stackrel{\circ P}{\longrightarrow} \mathcal{O}^{1 \times \ell_{1}} \text { exact } \Longleftrightarrow \forall z \in \mathbb{C}: \mathcal{O}_{z}^{1 \times \ell_{3}} \stackrel{\circ Q}{\longrightarrow} \mathcal{O}_{z}^{1 \times \ell_{2}} \stackrel{\circ P}{\longrightarrow} \mathcal{O}_{z}^{1 \times \ell_{1}} \\
& \text { exact } \underset{(31)}{\Longleftrightarrow} \forall z \in \mathbb{C}: \mathcal{E}_{\mathfrak{m}_{E}(z)}^{\prime 1 \times \ell_{3}} \stackrel{\circ Q}{\longrightarrow} \mathcal{E}_{\mathfrak{m}_{E}(z)}^{\prime 1 \times \ell_{2}} \stackrel{\circ P}{\longrightarrow} \mathcal{E}_{\mathfrak{m}_{E}(z)}^{\prime 1 \times \ell_{1}} \text { exact } \underset{\text { Thm. 4.4,(1) }}{\Longleftrightarrow} \\
& \forall z \in \mathbb{C}: \mathcal{P E}(-i z)^{\ell_{1}} \stackrel{P \circ}{\longrightarrow} \mathcal{P E}(-i z)^{\ell_{2}} \stackrel{Q \circ}{\longrightarrow} \mathcal{P E}(-i z)^{\ell_{3}} \text { exact } \Longleftrightarrow \\
& P \circ \mathcal{P} \mathcal{E}^{\ell_{1}}=\bigoplus_{z \in \mathbb{C}}\left(\mathcal{B}_{2} \bigcap \mathcal{P E}(-i z)^{\ell_{2}}\right)=\mathcal{B}_{2} \bigcap \mathcal{P} \mathcal{E}^{\ell_{2}} \Longrightarrow \operatorname{cl}_{E}\left(P \circ \mathcal{E}^{\ell_{1}}\right)=\mathcal{B}_{2} .
\end{aligned}
$$


Corollary 4.9. For every submodule $U \subseteq \mathcal{E}^{\prime 1 \times \ell}$, and $z \in \mathbb{C}$ as in (51) we obtain

$$
\begin{gathered}
U \subseteq \operatorname{cl}_{E}(U) \subseteq U_{\mathfrak{m}_{E}(z)}, U_{\mathfrak{m}_{E}(z)}=\operatorname{cl}_{E}(U)_{\mathfrak{m}_{E}(z)}, \mathcal{K} U=\mathcal{K} \mathrm{cl}_{E}(U)=\mathcal{K} U_{\mathfrak{m}_{E}(z)} \\
M_{\mathfrak{m}_{E}(z)}=\left(\mathcal{E}^{\prime 1 \times \ell} / U\right)_{\mathfrak{m}_{E}(z)}=\mathcal{E}_{\mathfrak{m}_{E}(z)}^{\prime 1 \times \ell} / U_{\mathfrak{m}_{E}(z)}=\left(\mathcal{E}^{\prime 1 \times \ell} / \mathrm{cl}_{E}(U)\right)_{\mathfrak{m}_{E}(z)} \\
\mathcal{K}^{1 \times \ell} / \mathcal{K} U=\mathcal{K} \otimes_{\mathcal{E}^{\prime}}\left(\mathcal{E}^{\prime 1 \times \ell} / U\right)=\mathcal{K} \otimes_{\mathcal{E}^{\prime}}\left(\mathcal{E}^{\prime 1 \times \ell} / \mathrm{cl}_{E}(U)\right)= \\
\mathcal{K} \otimes_{\mathcal{E}_{\mathfrak{m}_{E}(z)}^{\prime}}\left(\mathcal{E}_{\mathfrak{m}_{E}(z)}^{\prime 1 \times \ell} / U_{\mathfrak{m}_{E}(z)}\right)
\end{gathered}
$$

In the preceding Cor. 4.9 define $M:=\mathcal{E}^{\prime 1 \times \ell} / U$ and

$p:=\operatorname{rank}(U):=\operatorname{dim}_{\mathcal{K}}(\mathcal{K} U), m:=\operatorname{rank}(M):=\operatorname{dim}_{\mathcal{K}}\left(\mathcal{K} \otimes_{\mathcal{E}^{\prime}} M\right)$, hence

$$
p+m=\ell, \mathcal{K} \otimes_{\mathcal{E}^{\prime}} M \cong \mathcal{K}^{1 \times m} .
$$

Corollary and Definition 4.10. For the data from (55) the kernel of the canonical map $M \rightarrow \mathcal{K} \otimes_{\mathcal{E}^{\prime}} M, x \mapsto 1 \otimes x$, is the torsion submodule

$$
\operatorname{tor}(M):=\left\{x \in M ; \exists f \in \mathcal{E}^{\prime} \text { with } f \neq 0 \text { and } f x=0\right\}
$$

of $M$, and hence $M$ is a torsion module, i.e., $M=\operatorname{tor}(M)$, if and only if $\mathcal{K} \otimes_{\mathcal{E}^{\prime}} M=0$. Since $M$ is f.g. the canonical map maps $M$ into a free $\mathcal{E}^{\prime}$-submodule of $\mathcal{K}^{1 \times m}$ of dimension $m$ and hence there is a matrix

$$
\begin{gathered}
P \in \mathcal{E}^{\prime \ell \times m} \text { such that } U P=0, \text { hence } U \subseteq U_{2}:=\operatorname{ker}(\circ P)=\left\{\xi \in \mathcal{E}^{\prime \times \ell} ; \xi P=0\right\}, \\
\text { and } \operatorname{tor}(M)=U_{2} / U=\operatorname{ker}\left((\circ P)_{\text {ind }}: M=\mathcal{E}^{\prime 1 \times \ell} / U \rightarrow \mathcal{E}^{\prime 1 \times m}, \xi+U \mapsto \xi P\right) \\
\text { and } \mathcal{E}^{\prime 1 \times m} / \mathcal{E}^{\prime 1 \times \ell} P \text { is a torsion module. }
\end{gathered}
$$

The submodule $U_{2}$ is closed and hence tor $(M)$ is closed in $M=\mathcal{E}^{\prime 1 \times \ell} / U$.

\section{Autonomous behaviors}

The principal goal of this section is the proof of Thms. 5.6 and 5.8.

Let, as usual, $\mathcal{D}:=\mathcal{D}(\mathbb{R})$ denote the subspace of $\mathcal{E}$ of smooth functions with compact support with its standard topology, $\mathcal{D}^{\prime}=\mathcal{D}^{\prime}(\mathbb{R}, \mathbb{C})$ its dual space of distributions and $\mathcal{D}_{+}^{\prime}$ the subspace of $\mathcal{D}^{\prime}$ of distributions with left bounded support. The convolution product makes $\mathcal{D}_{+}^{\prime}$ an integral domain [31, Thm. VI.XIV] and $\mathcal{E}^{\prime}$ is a subalgebra of $\mathcal{D}_{+}^{\prime}$. The subspace $\mathcal{E}_{+}=\mathcal{D}_{+}^{\prime} \bigcap \mathcal{E}$ of smooth functions with left bounded support is an ideal of $\mathcal{D}_{+}^{\prime}$ and therefore a torsionfree $\mathcal{D}_{+}^{\prime}$-module. We conclude that $\mathcal{E}_{+}$is a torsionfree $\mathcal{E}^{\prime}$-submodule of $\mathcal{E}^{\prime} \mathcal{E}$.

Theorem and Definition 5.1. (cf. [17, Prop. 3.10] [35, Def. 2, Prop. 4]) For a not necessarily closed submodule $U \subseteq \mathcal{E}^{\prime 1 \times \ell}$ and the associated f.g. $\mathcal{E}^{\prime}$-module $M:=$ $\mathcal{E}^{\prime 1 \times \ell} / U$ and gen. beh. $\mathcal{B}:=U^{\perp}$ the following properties are equivalent:

1. $M$ is a torsion module, i.e., there is a nonzero $f \in \mathcal{E}^{\prime}$ with $f M=0$ or $\mathcal{E}^{1 \times \ell} f \subseteq U$.

2. $\mathcal{B} \cap \mathcal{E}_{+}^{\prime \ell}=0$, i.e., the past of a trajectory of $\mathcal{B}$ determines its future.

3. There is a nonzero $g \in \mathcal{E}^{\prime}$ with

$$
g \circ \mathcal{B}=0 \text { or } \mathcal{B} \subseteq\left(\mathcal{E}^{1 \times \ell} g\right)^{\perp}=\left\{w \in \mathcal{E}^{\ell} ; g \circ w=0\right\} .
$$

If $U$ is closed one may choose $f=g$ in (1) and (3).

Under these conditions $\mathcal{B}$ is called autonomous. 
Proof. 1. $\Longrightarrow$ 3.: The inclusion

$\mathcal{E}^{\prime 1 \times \ell} f \subseteq U$ implies $\mathcal{B} \subseteq\left(\mathcal{E}^{\prime 1 \times \ell} f\right)^{\perp}=\left\{w \in \mathcal{E}^{\ell} ; f \circ w=0\right\} \Longrightarrow f \circ \mathcal{B}=0$.

3. $\Longrightarrow 1 .:$ The equation $g \circ \mathcal{B}=0,0 \neq g$, implies

$$
\begin{gathered}
U^{\perp} \subseteq\left(\mathcal{E}^{1 \times \ell} g\right)^{\perp} \Longrightarrow \mathcal{E}^{1 \times \ell} g \subseteq\left(\mathcal{E}^{1 \times \ell} g\right)^{\perp \perp} \subseteq U^{\perp \perp}=\operatorname{cl}_{E}(U) \Longrightarrow \\
g M^{\prime}=0 \text { for } M^{\prime}:=\mathcal{E}^{\prime 1 \times \ell} / \operatorname{cl}_{E}(U) .
\end{gathered}
$$

Thus $M^{\prime}$ is a torsion module and so is $M$ due to (54).

1. $\Longrightarrow 2 .:$ The module $\mathcal{B} \cap \mathcal{E}_{+}^{\ell}$ is a submodule of the torsionfree $\mathcal{E}^{\prime}$-module $\mathcal{E}_{+}^{\ell}$ and annihilated by the nonzero $f$ and thus zero.

2. $\Longrightarrow 1 .:$ Recall $\mathcal{K}=$ quot $\left(\mathcal{E}^{\prime}\right)$. We give an indirect proof and assume that $M$ is not a torsion module or $\mathcal{K} \otimes_{\mathcal{E}^{\prime}} M \neq 0$. For the data from (57) this implies

$$
\begin{aligned}
& m>0 \text { and }(\circ P)_{\text {ind }} \neq 0 \text { or } U \subseteq U_{2}=\operatorname{cl}_{E}\left(U_{2}\right) \subsetneq \mathcal{E}^{\prime 1 \times \ell} \Longrightarrow \\
& 0 \neq \mathcal{B}_{2}:=U_{2}^{\perp} \subseteq \mathcal{B} \text {. Since } \mathcal{B} \bigcap \mathcal{E}_{+}^{\ell}=0 \text { also } \mathcal{B}_{2} \bigcap \mathcal{E}_{+}^{\ell}=0 .
\end{aligned}
$$

From (57) and (46) we infer $\operatorname{cl}_{E}\left(P \circ \mathcal{E}^{m}\right)=\mathcal{B}_{2} \neq 0$ and hence $P \circ \mathcal{E}^{m} \neq 0$. Finally $\mathcal{D}$ and therefore $\mathcal{E}_{+}$are dense in $\mathcal{E}$ and therefore

$$
0 \neq P \circ \mathcal{E}^{m}=P \circ \operatorname{cl}_{E}\left(\mathcal{E}_{+}^{m}\right) \subseteq \operatorname{cl}_{E}\left(P \circ \mathcal{E}_{+}^{m}\right) \subseteq \mathcal{B}_{2} \bigcap \mathcal{E}_{+}^{\ell}=0
$$

This is a contradiction.

If an ideal $\mathfrak{a}$ of $\mathcal{E}^{\prime}$ is not closed and

$$
g \in \operatorname{cl}_{E}(\mathfrak{a}) \backslash \mathfrak{a} \text { then } \mathfrak{a}^{\perp}=\operatorname{cl}_{E}(\mathfrak{a})^{\perp}, g \circ \mathfrak{a}^{\perp}=0 \text {, but } g\left(\mathcal{E}^{\prime} / \mathfrak{a}\right) \neq 0 .
$$

So, in general, the distributions $f$ and $g$ in the preceding theorem cannot be chosen equal.

There is no structure theorem for arbitrary closed $\mathcal{E}^{\prime}$-submodules $U \subseteq \mathcal{E}^{1 \times \ell}$ or gen. beh. $\mathcal{B}=U^{\perp}$, but we construct all autonomous gen. beh. in the next theorem. So assume

$$
\begin{gathered}
U \subseteq \mathcal{E}^{1 \times \ell}, M:=\mathcal{E}^{1 \times \ell} / U, 0 \neq f \in \mathcal{E}^{\prime}, \\
f M=0 \text { or } \mathcal{E}^{1 \times \ell} f \subseteq U, \mathcal{B}:=U^{\perp} \subseteq\left(\mathcal{E}^{1 \times \ell} f\right)^{\perp}=\mathcal{B}_{f}^{\ell}, \\
\text { where } \mathcal{B}_{f}:=\left(\mathcal{E}^{\prime} f\right)^{\perp}=\{w \in \mathcal{E} ; f \circ w=0\} .
\end{gathered}
$$

We use data from (32) and (33), especially $V:=V_{\mathbb{C}}(f)$, for $f$ and the (Stein) algebra isomorphism (34)

$$
\mathcal{O} / \mathcal{O} f \cong \prod_{z \in V} \mathcal{O} / \mathcal{O}(s-z)^{k(z)}, g+\mathcal{O} f \mapsto\left(g+\mathcal{O}(s-z)^{k(z)}\right)_{z \in V}
$$

This isomorphism immediately implies

Corollary 5.2. There are elements $\epsilon_{z} \in \mathcal{O}, z \in V$, unique mod $\mathcal{O} f$, such that

$$
\begin{gathered}
\forall z, z^{\prime} \in V: \epsilon_{z} \equiv \delta_{z, z^{\prime}} \bmod \mathcal{O}\left(s-z^{\prime}\right)^{k\left(z^{\prime}\right)}, \text { hence } \\
\forall z, z^{\prime} \in V: \epsilon_{z} \epsilon_{z^{\prime}} \equiv \delta_{z, z^{\prime}} \epsilon_{z} \bmod \mathcal{O} f .
\end{gathered}
$$

In particular, the $\epsilon_{z}+\mathcal{O} f, z \in V$, are orthogonal idempotents in $\mathcal{O} / \mathcal{O} f$. 
Proof. $\epsilon_{z}+\mathcal{O} f$ is the unique inverse image under (60) of $\left(\delta_{z, z^{\prime}}+\mathcal{O}\left(s-z^{\prime}\right)^{k\left(z^{\prime}\right)}\right)_{z^{\prime} \in V}$.

The ideas for the following construction of distributions $\epsilon_{z} \in \mathcal{E}^{\prime}$ that satisfy the congruences in (61) were extracted from [30, pp. 882-, (24)-(29)], but not trivially: Recall

$$
f=(s-z)^{k(z)} f^{z} \text { with } f^{z} \in \mathcal{E}^{\prime}, f^{z}(z) \neq 0, k(z):=\operatorname{mult}(f, z) .
$$

The function $f^{-1}$ is meromorphic with poles at $z \in V$. Let $\operatorname{pp}_{z}\left(f^{-1}\right)$ denote its principal part at $z$ which is given and characterized by

$$
\begin{gathered}
\operatorname{pp}_{z}\left(f^{-1}\right)=\sum_{i=1}^{k(z)} a_{z, i}(s-z)^{-i}, a_{z, i} \in \mathbb{C}, g^{z}:=f^{-1}-\operatorname{pp}_{z}\left(f^{-1}\right) \in \mathcal{O}_{z} . \text { Define } \\
\epsilon_{z}:=f \operatorname{pp}_{z}\left(f^{-1}\right)=f^{z}(s-z)^{k(z)} \operatorname{pp}_{z}\left(f^{-1}\right)=f^{z} \sum_{i=1}^{k(z)} a_{z, i}(s-z)^{k(z)-i} \in \mathcal{E}^{\prime}
\end{gathered}
$$

In particular, the action $\epsilon_{z} \circ w \in \mathcal{E}$ is defined for all $w \in \mathcal{E}$.

Lemma 5.3. The $\epsilon_{z} \in \mathcal{E}^{\prime}, z \in V$, from (63) satisfy the congruences

$$
\begin{gathered}
\epsilon_{z} \equiv \delta_{z, z^{\prime}} \quad \bmod \mathcal{E}^{\prime}\left(s-z^{\prime}\right)^{k\left(z^{\prime}\right)}, z^{\prime} \in V, \text { or } \\
\epsilon_{z}-1 \in \mathcal{E}^{\prime}(s-z)^{k(z)} \text { and } \epsilon_{z} \in \bigcap_{z^{\prime} \in V, z^{\prime} \neq z} \mathcal{E}^{\prime}\left(s-z^{\prime}\right)^{k\left(z^{\prime}\right)} .
\end{gathered}
$$

Proof. (i)

$$
\begin{gathered}
1=f f^{-1}=f\left(\operatorname{pp}_{z}\left(f^{-1}\right)+\left(f^{-1}-\operatorname{pp}_{z}\left(f^{-1}\right)\right)\right)=\epsilon_{z}+f g^{z} \Longrightarrow \\
f g^{z}=1-\epsilon_{z}=(s-z)^{k(z)} f^{z} g^{z} \in \mathcal{O} \underset{f^{z} \bar{g}^{z} \in \mathcal{O}_{z}}{\Longrightarrow} f^{z} g^{z} \in \mathcal{O} \Longrightarrow \\
1-\epsilon_{z}=(s-z)^{k(z)}\left(f^{z} g^{z}\right) \in \mathcal{E}^{\prime} \bigcap \mathcal{O}(s-z)^{k(z)}=\mathcal{E}^{\prime}(s-z)^{k(z)} .
\end{gathered}
$$

(ii) For $z \neq z^{\prime}$ there is another product representation

$$
\begin{gathered}
f=(s-z)^{k(z)}\left(s-z^{\prime}\right)^{k\left(z^{\prime}\right)} f^{z, z^{\prime}}, f^{z, z^{\prime}} \in \mathcal{E}^{\prime}, f^{z}=\left(s-z^{\prime}\right)^{k\left(z^{\prime}\right)} f^{z, z^{\prime}} \Longrightarrow \\
\epsilon_{z}=f^{z} \sum_{i=1}^{k(z)} a_{z, i}(s-z)^{k(z)-i}=\left(s-z^{\prime}\right)^{k\left(z^{\prime}\right)} f^{z, z^{\prime}} \sum_{i=1}^{k(z)} a_{z, i}(s-z)^{k(z)-i} \in \\
\mathcal{E}^{\prime} \bigcap \mathcal{O}\left(s-z^{\prime}\right)^{k\left(z^{\prime}\right)}=\mathcal{E}^{\prime}\left(s-z^{\prime}\right)^{k\left(z^{\prime}\right)} .
\end{gathered}
$$

If (cf. (18))

$f \in \mathcal{O}_{a, p} \subset \mathcal{E}^{\prime}$ then also $\forall z \in V \forall i=1, \cdots, k(z):(s-z)^{-i} f \in \mathcal{O}_{a, p} \Longrightarrow$

$$
\forall z \in V: \epsilon_{z}=\sum_{i=1}^{k(z)} a_{z, i}(s-z)^{-i} f \in \mathcal{O}_{a, p} .
$$


The condition $f^{z}(z) \neq 0$ in $f=(s-z)^{k(z)} f^{z}$ implies that $f^{z}$ is a unit in $\mathcal{E}_{\mathfrak{m}_{E}(z)}^{\prime}$ and hence $\mathcal{E}_{\mathfrak{m}_{E}(z)}^{\prime} f=\mathcal{E}_{\mathfrak{m}_{E}(z)}^{\prime}(s-z)^{k(z)}$. For $z \notin V$ we get $k(z)=0, f=f^{z}$ and $\mathcal{E}_{\mathfrak{m}_{E}(z)}^{\prime} f=\mathcal{E}_{\mathfrak{m}_{E}(z)}^{\prime}$. From (14), (15), (48) and $s=i \partial$ we know that

$$
\begin{aligned}
& \mathcal{B}_{f} \bigcap \mathcal{P} \mathcal{E}=\bigoplus_{z \in \mathbb{C}}\left(\mathcal{B}_{f} \bigcap \mathcal{P E}(-i z t)\right) \text { and } \\
& \mathcal{B}_{f} \bigcap \mathcal{P E}(-i z t) \underset{\text { ident. }}{=} \operatorname{Hom}_{\mathcal{E}_{\mathfrak{m}_{E}(z)}^{\prime}}\left(\mathcal{E}_{\mathfrak{m}_{E}(z)}^{\prime} / \mathcal{E}_{\mathfrak{m}_{E}(z)}^{\prime} f, \mathcal{P E}(-i z)\right)= \\
& \left.\operatorname{Hom}_{\mathcal{E}_{\mathfrak{m}_{E}(z)}^{\prime}}^{\prime}\left(\mathcal{E}_{\mathfrak{m}_{E}(z)}^{\prime} / \mathcal{E}_{\mathfrak{m}_{E}(z)}^{\prime}(s-z)^{k(z)}, \mathcal{P} \mathcal{E}(-i z)\right)\right) \underset{\text { ident. }}{=}\left(\mathcal{E}^{\prime}(s-z)^{k(z)}\right)^{\perp}= \\
& \operatorname{ann}_{\mathcal{E}}\left((s-z)^{k(z)}\right)=\mathbb{C}[t]_{<k(z)} e^{-i z t} \Longrightarrow \\
& \mathcal{B}_{f} \bigcap \mathcal{P} \mathcal{E}=\bigoplus_{z \in \mathbb{C}} \mathbb{C}[t]_{<k(z)} e^{-i z t}=\bigoplus_{z \in V} \mathbb{C}[t]_{<k(z)} e^{-i z t} .
\end{aligned}
$$

Corollary 5.4. For all $z, z^{\prime} \in V$ and $w_{z^{\prime}} \in\left(\mathcal{E}^{\prime}\left(s-z^{\prime}\right)^{k\left(z^{\prime}\right)}\right)^{\perp}=\mathbb{C}[t]_{<k\left(z^{\prime}\right)} e^{-i z^{\prime} t}$, i.e., $\left(s-z^{\prime}\right)^{k\left(z^{\prime}\right)} \circ w_{z^{\prime}}=0$ we have

$$
\epsilon_{z} \circ w_{z^{\prime}}=\delta_{z, z^{\prime}} w_{z^{\prime}} \text {, hence } \epsilon_{z} \circ\left(\mathcal{B}_{f} \bigcap \mathcal{P E}\right)=\mathbb{C}[t]_{<k(z)} e^{-i z t} .
$$

Proof. For $z, z^{\prime} \in V$ we infer from Lemma 5.3 that

$$
\begin{gathered}
\epsilon_{z}=\delta_{z, z^{\prime}}+g\left(s-z^{\prime}\right)^{k\left(z^{\prime}\right)}, g \in \mathcal{E}^{\prime} \Longrightarrow \\
\epsilon_{z} \circ w_{z^{\prime}}=\delta_{z, z^{\prime}} w_{z^{\prime}}+g \circ\left(s-z^{\prime}\right)^{k\left(z^{\prime}\right)} \circ w_{z^{\prime}} \underset{\left(s-z^{\prime}\right)^{k\left(z^{\prime}\right)}}{=}{ }_{o w_{z^{\prime}}=0} \delta_{z, z^{\prime}} w_{z^{\prime}} .
\end{gathered}
$$

The inclusion $\mathcal{B}=U^{\perp} \subseteq \mathcal{B}_{f}^{\ell}$ and the preceding considerations also furnish

Corollary 5.5. For the behavior $\mathcal{B}=U^{\perp} \subseteq \mathcal{B}_{f}^{\ell}$ and $z \in V$ there holds the decomposition

$$
\begin{gathered}
\mathcal{B} \bigcap \mathcal{P \mathcal { E } ^ { \ell }}=\bigoplus_{z^{\prime} \in V}\left(\mathcal{B} \bigcap \mathcal{P E}\left(-i z^{\prime}\right)^{\ell}\right)=\bigoplus_{z^{\prime} \in V}\left(\mathcal{B} \bigcap \mathbb{C}[t]_{<k\left(z^{\prime}\right)}^{\ell} e^{-i z^{\prime} t}\right) \text { and } \\
\epsilon_{z} \circ: \bigoplus_{z^{\prime} \in V}\left(\mathcal{B} \bigcap \mathbb{C}[t]_{<k\left(z^{\prime}\right)}^{\ell} e^{-i z^{\prime} t}\right) \rightarrow \mathcal{B} \bigcap \mathbb{C}[t]_{<k(z)}^{\ell} e^{-i z t}, \sum_{z^{\prime} \in V} w_{z^{\prime}} \mapsto w_{z},
\end{gathered}
$$

is the projection.

Theorem 5.6. 1. For the data from (59)-(69) there are polynomial matrices $R^{z} \in$ $\mathbb{C}[s]^{\ell \times \ell}, z \in V:=V_{\mathbb{C}}(f)$, of degree $\operatorname{deg}_{s}\left(R^{z}\right)<k(z)$ such that for all $z \in V$

$$
\begin{gathered}
\mathcal{B} \bigcap \mathcal{P E}(-i z)^{\ell}=\left\{w \in \mathbb{C}[t]_{<k(z)}^{\ell} e^{-i z t} ; R^{z} \circ w=0\right\}= \\
\left\{w \in \mathcal{E}^{\ell} ;(s-z)^{k(z)} \circ w=0, R^{z} \circ w=0\right\}=\left(\mathcal{E}^{\prime 1 \times 2 \ell}\left(\begin{array}{c}
R^{z} \\
(s-z)^{k(z)} \text { id }_{\ell}
\end{array}\right)\right)^{\perp}
\end{gathered}
$$

2. Conversely, for given $f \neq 0$ in $\mathcal{E}^{\prime}$ with the data from (33) and (34) choose arbitrary polynomial matrices $R^{z} \in \mathbb{C}[s]^{\ell \times \ell}, z \in V$, and define

$$
\begin{gathered}
U(z):=\mathcal{E}^{\prime 1 \times \ell} R^{z}+\mathcal{E}^{\prime 1 \times \ell}(s-z)^{k(z)}=\mathcal{E}^{\prime 1 \times 2 \ell}\left(\begin{array}{c}
R^{z} \\
(s-z)^{k(z)} \mathrm{id}_{\ell}
\end{array}\right) \subseteq \mathcal{E}^{\prime 1 \times \ell}, \\
\text { hence } U(z)^{\perp} \subset \mathbb{C}[t]_{<k(z)}^{\ell} e^{-i z t} \subset \mathcal{P} \mathcal{E}(-i z)^{\ell}, z \in V_{\mathbb{C}}(f),
\end{gathered}
$$


where the $U(z)$ are closed and the $U(z)^{\perp}$ are $\mathbb{C}$-f.d. differential behaviors. Then $\oplus_{z \in V} U(z)^{\perp}\left(\subseteq \bigoplus_{z \in V} \mathbb{C}[t]_{<k(z)}^{\ell} e^{-i z t}\right)$ is an $\mathcal{E}^{\prime}$-submodule of $\mathcal{E}^{\ell}$ and

$$
\mathcal{B}:=\operatorname{cl}_{E}\left(\oplus_{z \in V_{\mathbb{C}}(f)} U(z)^{\perp}\right)
$$

is the unique autonomous gen. beh. $\mathcal{B}$ with

$$
\mathcal{B} \bigcap \mathcal{P E}(-i z)^{\ell}= \begin{cases}U(z)^{\perp} & \text { if } z \in V \\ 0 & \text { if } z \in \mathbb{C} \backslash V .\end{cases}
$$

In particular, it is annihilated by $f$, i.e., $f \circ \mathcal{B}=0$.

3. In the situation of 1. or 2. and with the $\epsilon_{z} \in \mathcal{E}^{\prime}$ from (63) the map

$$
\mathcal{B} \rightarrow \prod_{z \in V} U(z)^{\perp}, w \mapsto\left(\epsilon_{z} \circ w\right)_{z \in V}
$$

is injective.

Proof. 1. For $z \in V$ equation (48) furnishes

$$
\mathcal{B} \bigcap \mathcal{P E}(-i z)^{\ell} \underset{\text { ident. }}{=} \operatorname{Hom}_{\mathcal{E}_{\mathfrak{m}_{E}(z)}^{\prime}}\left(\mathcal{E}_{\mathfrak{m}_{E}(z)}^{\prime 1 \times \ell} / U_{\mathfrak{m}_{E}(z)}, \mathcal{P E}(-i z)\right) .
$$

Recall that in $f=(s-z)^{k(z)} f^{z}$ the distribution $f^{z}$ is a unit in $\mathcal{E}_{\mathfrak{m}_{E}(z)}^{\prime}$. Together with $f \mathcal{E}^{\prime 1 \times \ell} \subseteq U$ this yields

$$
\begin{gathered}
\mathcal{E}_{\mathfrak{m}_{E}(z)}^{\prime \times \ell} f=\mathcal{E}_{\mathfrak{m}_{E}(z)}^{\prime \times \ell}(s-z)^{k(z)} \subseteq U_{\mathfrak{m}_{E}(z)} \Longrightarrow U_{\mathfrak{m}_{E}(z)} / \mathcal{E}_{\mathfrak{m}_{E}(z)}^{\prime 1 \times \ell}(s-z)^{k(z)} \subseteq \\
\mathcal{E}_{\mathfrak{m}_{E}(z)}^{\prime 1 \times \ell} / \mathcal{E}_{\mathfrak{m}_{E}(z)}^{\prime 1 \times \ell}(s-z)^{k(z)} \underset{(29)}{\cong} \mathbb{C}[s]^{1 \times \ell} / \mathbb{C}[s]^{1 \times \ell}(s-z)^{k(z)} \\
\text { where } \mathbb{C}[s] / \mathbb{C}[s](s-z)^{k(z)}=\bigoplus_{i=0}^{k(z)-1} \mathbb{C}\left(s^{i}+\mathbb{C}[s](s-z)^{k(z)}\right) .
\end{gathered}
$$

But all $\mathbb{C}[s]$-submodules of $\mathbb{C}[s]^{1 \times \ell} / \mathbb{C}[s]^{1 \times \ell}(s-z)^{k(z)}$ are of the form

$$
\begin{aligned}
& \left(\mathbb{C}[s]^{1 \times \ell} R^{z}+\mathbb{C}[s]^{1 \times \ell}(s-z)^{k(z)}\right) / \mathbb{C}[s]^{1 \times \ell}(s-z)^{k(z)} \text { with } \\
& R^{z} \in \mathbb{C}[s]^{\ell \times \ell}, \operatorname{deg}_{s}\left(R^{z}\right)<k(z) \underset{(77)}{\Longrightarrow} U_{\mathfrak{m}_{E}(z)}=\mathcal{E}_{\mathfrak{m}_{E}(z)}^{\prime 1 \times \ell} R^{z}+\mathcal{E}_{\mathfrak{m}_{E}(z)}^{\prime 1 \times \ell}(s-z)^{k(z)} \overrightarrow{(76)} \\
& \mathcal{B} \bigcap \mathcal{P E}(-i z)^{\ell} \underset{\text { ident }}{=} \\
& \operatorname{Hom}_{\mathcal{E}_{\mathfrak{m}_{E}(z)}^{\prime}}\left(\mathcal{E}_{\mathfrak{m}_{E}(z)}^{\prime 1 \times \ell} /\left(\mathcal{E}_{\mathfrak{m}_{E}(z)}^{\prime 1 \times \ell} R^{z}+\mathcal{E}_{\mathfrak{m}_{E}(z)}^{\prime 1 \times \ell}(s-z)^{k(z)}\right), \mathcal{P} \mathcal{E}(-i z)\right)= \\
& \left(\mathcal{E}^{\prime 1 \times 2 \ell}\left(\underset{(s-z)^{k(z)} \mathrm{id}_{\ell}}{R^{z}}\right)\right)^{\perp} \text {. }
\end{aligned}
$$

2. The module $U(z)$ is closed since $U(z) / \mathcal{E}^{1 \times \ell}(s-z)^{k(z)}$ is a (closed) subspace of the $\mathbb{C}$-f.d. Hausdorff space $\left(\mathcal{E} / \mathcal{E}^{\prime}(s-z)^{k(z)}\right)^{1 \times \ell}$ (cf. (29)). This implies that

$$
\begin{aligned}
U(z)^{\perp}= & \left\{w \in \mathcal{E}^{\ell} ; R^{z} \circ w=0,(s-z)^{k(z)} \circ w=0\right\}= \\
& \left\{w \in \mathbb{C}[t]_{<k(z)}^{\ell} e^{-i z t} ; R^{z} \circ w=0\right\}
\end{aligned}
$$


is a $\mathbb{C}$-f.d. behavior. Then $\mathcal{B}_{1}:=\oplus_{z \in V} U(z)^{\perp}$ is an $\mathcal{E}^{\prime}$-submodule of $\mathcal{E}^{\ell}$ and its closure $\mathcal{B}:=\operatorname{cl}_{E}\left(\mathcal{B}_{1}\right)$ is a gen. beh.. Recall that $f \circ: \mathcal{E}^{\ell} \rightarrow \mathcal{E}^{\ell}$ is continuous. From

$$
\begin{gathered}
f=(s-z)^{k(z)} f^{z} \text { and }(s-z)^{k(z)} \circ U(z)^{\perp}=0 \text { we infer } f \circ \mathcal{B}_{1}=0 \Longrightarrow \\
f \circ \mathcal{B}=f \circ \operatorname{cl}_{E}\left(\mathcal{B}_{1}\right) \subseteq \operatorname{cl}_{E}\left(f \circ \mathcal{B}_{1}\right)=\operatorname{cl}_{E}(0)=0 \Longrightarrow f \circ \mathcal{B}=0 \underset{\text { Cor. 5.5 }}{\Longrightarrow} \\
\mathcal{B} \bigcap \mathcal{P} \mathcal{E}^{\ell}=\bigoplus_{z \in V}\left(\mathcal{B} \bigcap \mathbb{C}[t]_{<k(z)}^{\ell} e^{-i z t}\right) \text { and } \\
U(z)^{\perp}=\left\{w \in \mathbb{C}[t]_{<k(z)}^{\ell} e^{-i z t} ; R^{z} \circ w=0\right\} \subseteq \mathcal{B} \bigcap \mathbb{C}[t]_{<k(z)}^{\ell} e^{-i z t} .
\end{gathered}
$$

It remains to show that the last inclusion is an equality. So assume $w \in \mathcal{B} \cap \mathbb{C}[t]_{<k(z)}^{\ell} e^{-i z t}$. Since $\mathcal{B}$ is the closure of $\mathcal{B}_{1}$ we conclude that

$$
w=\lim _{n \rightarrow \infty} w_{n} \text { where } w_{n}=\sum_{z \in V} w_{n, z}, w_{n, z} \in \mathbb{C}[t]_{<k(z)}^{\ell} e^{-i z t}, R^{z} \circ w_{n, z}=0 .
$$

Notice that for fixed $n$ only finitely many $w_{n, z}$ are nonzero. Recall that $\epsilon_{z} \circ$ and $R^{z} \circ$ are continuous. Cor. 5.4 furnishes

$$
\begin{gathered}
\epsilon_{z} \circ w=w, \epsilon_{z} \circ w_{n}=w_{n, z} \Longrightarrow w=\epsilon_{z} \circ w=\epsilon_{z} \circ \lim _{n} w_{n}= \\
\lim _{n}\left(\epsilon_{z} \circ w_{n}\right)=\lim _{n} w_{n, z} \text { and } R^{z} \circ w=\lim _{n} R^{z} \circ w_{n, z}=\lim _{n} 0=0 \Longrightarrow \\
w \in\left\{v \in \mathbb{C}[t]_{<k(z)}^{\ell} e^{-i z t} ; R^{z} \circ v=0\right\}=U(z)^{\perp} \Longrightarrow U(z)^{\perp}=\mathcal{B} \bigcap \mathbb{C}[t]_{<k(z)}^{\ell} e^{-i z t} .
\end{gathered}
$$

3. Under the assumption $w \in \mathcal{B}$ and $\epsilon_{z} \circ w=0$ for all $z \in V$ we have to prove $w=0$. Let $\mathcal{B}_{w}:=\operatorname{cl}_{E}\left(\mathcal{E}^{\prime} \circ w\right) \ni w$. From Cor. 5.5 we get

$$
\begin{aligned}
& \mathcal{B}_{w} \bigcap \mathcal{P} \mathcal{E}^{\ell}=\bigoplus_{z \in V}\left(\mathcal{B}_{w} \bigcap \mathcal{P E}(-i z)^{\ell}\right) \text { and } \forall z \in V: \mathcal{B}_{w} \bigcap \mathcal{P E}(-i z)^{\ell}= \\
& \epsilon_{z} \circ\left(\mathcal{B}_{w} \bigcap \mathcal{P \mathcal { E } ^ { \ell }}\right) \subseteq \epsilon_{z} \circ \mathrm{cl}_{E}\left(\mathcal{E}^{\prime} \circ w\right) \subseteq \mathrm{cl}_{E}\left(\mathcal{E}^{\prime} \circ \epsilon_{z} \circ w\right)=\operatorname{cl}_{E}(0)=0 \Longrightarrow \\
& \mathcal{B}_{w} \bigcap \mathcal{P \mathcal { E } ^ { \ell }}=0 \Longrightarrow \mathcal{B}_{w}=\operatorname{cl}_{E}\left(\mathcal{B}_{w} \bigcap \mathcal{P \mathcal { E } ^ { \ell }}\right)=0 \Longrightarrow w=0 .
\end{aligned}
$$

Remark 5.7. Consider the special case $\ell=1,0 \neq f \in \mathcal{E}^{\prime}$ and $\mathcal{B}:=\left(\mathcal{E}^{\prime} f\right)^{\perp}=$ $\{w \in \mathcal{E} ; f \circ w=0\}$ in Thm. 5.6. In this case the injectivity of the map in (75) was established in Schwartz' fundamental theorem [30, Thm. 9] from which he inferred that $\mathcal{B} \cap \mathcal{P E}$ is dense in $\mathcal{B}$ whereas we simply used Result 2.1. Moreover the quoted analysts proved a much stronger version of Thm. 5.6,(3), for the case $\ell=1$ and showed that for all $w \in \mathcal{B}_{f}$ and not only for $w \in \mathcal{B}_{f} \cap \mathcal{P E}=\bigoplus_{z \in V} \mathbb{C}[t]_{<k(z)} e^{-i z t}$ the convergent sum representation $w=\sum_{z \in V} \epsilon_{z} \circ w, V:=V_{\mathbb{C}}(f)$, holds where a suitable notion of convergence has to be used [30, Thm. 11], [2, Thms. 2,3, 4]. In [2] the authors make the essential assumption that $f$ is invertible, i.e., that $\mathcal{E}^{\prime} f$ is closed or $f \circ \mathcal{E}=\mathcal{E}$. Analogous convergent sum representations hold for arbitrary trajectories $w$ of $\mathcal{B}_{f}^{\ell}$ from (59) by componentwise application of the case $\ell=1$. The sum representations $w=\sum_{z \in V} \epsilon_{z} \circ w$ generalize the convergent Fourier series. Notice 
that for $w \in \mathcal{B}$ and $z \in V$

$$
\begin{gathered}
\epsilon_{z} \circ w \in \mathbb{C}[t]_{<k(z)} e^{-i z t}, \text { i.e., } \epsilon_{z} \circ w=\sum_{j=0}^{k(z)-1} a_{z, j} t^{j}, a_{z, j} \in \mathbb{C} \Longrightarrow \\
w=\sum_{z \in V} \epsilon_{z} \circ w=\sum_{z \in V, 0 \leq j<k(z)} a_{z, j} t^{j} e^{-i z t} .
\end{gathered}
$$

For

$$
f=\delta_{2 \pi}-\delta_{0} \underset{\text { ident. }}{=} \widehat{\delta_{2 \pi}}-\widehat{\delta_{0}}=e^{-2 \pi i s}-1, V=\mathbb{Z}
$$

all multiplicities $k(n), n \in V=\mathbb{Z}$, are 1 and therefore (78) obtains the standard form $w=\sum_{n \in \mathbb{Z}} a_{n} e^{i n t}$ of the Fourier series for any periodic $w \in \mathcal{E}$ with $f \circ w=$ $w(t+2 \pi)-w(t)=0$

Theorem 5.8. Every autonomous gen. beh. $\mathcal{B} \subseteq \mathcal{E}^{\ell}$ is indeed a behavior. If $0 \neq f \in \mathcal{E}^{\prime}$ with $\operatorname{supp}(f) \subseteq[-a, a], a>0$, annihilates $\mathcal{B}$, i.e., if $f \circ \mathcal{B}=0$, then there is a matrix $R \in \mathcal{E}^{\prime \ell \times \ell}$ with $\operatorname{supp}(R) \subseteq[-a, a]$ such that

$$
\mathcal{B}=\left\{w \in \mathcal{E}^{\ell} ; R \circ w=0, f \circ w=0\right\}, \text { hence } \mathcal{B}^{\perp}=\operatorname{cl}_{E}\left(\mathcal{E}^{\prime 1 \times 2 \ell}\left(\underset{f \mathrm{id}_{\ell}}{R}\right)\right) .
$$

Proof. We use Thm. 5.6 and its notations and obtain

$$
\begin{gathered}
\mathcal{B} \bigcap \mathcal{P} \mathcal{E}^{\ell}=\bigoplus_{z \in V}\left(\mathcal{B} \bigcap \mathcal{P E}(-i z)^{\ell}\right) \text { with } \\
\mathcal{B} \bigcap \mathcal{P E}(-i z)^{\ell}=\left\{w \in \mathbb{C}[t]_{<k(z)}^{\ell} e^{-i z t} ; R^{z} \circ w=0\right\}, R^{z} \in \mathbb{C}[s]^{\ell \times \ell} .
\end{gathered}
$$

For $z \in V$ we use $\epsilon_{z}=(s-z)^{-k(z)} f \sum_{j=1}^{k(z)} a_{z, j}(s-z)^{k(z)-j}$ from (63). By polynomial division with remainder we get

$$
\begin{gathered}
\sum_{j=1}^{k(z)} a_{z, j}(s-z)^{k(z)-j} R^{z}=P^{z}+(s-z)^{k(z)} Q^{z}, P^{z}, Q^{z} \in \mathbb{C}[s]^{\ell \times \ell} \\
\text { with } \operatorname{deg}_{s}\left(P^{z}\right)<k(z), P^{z}=\sum_{j=1}^{k(z)} P^{z, j}(s-z)^{k(z)-j}, P^{z, j} \in \mathbb{C}^{\ell \times \ell} \Longrightarrow \\
\epsilon_{z} R^{z}=(s-z)^{-k(z)} f \sum_{j=1}^{k(z)} a_{z, j}(s-z)^{k(z)-j} R^{z}=F^{z}+f Q^{z} \text { where } \\
F^{z}:=f \sum_{j=1}^{k(z)} P^{z, j}(s-z)^{-j} .
\end{gathered}
$$

By assumption $f \in \mathcal{E}^{\prime}$ has its support in $[-a, a]$ is therefore contained in some $\mathcal{O}_{a, p}, p \in \mathbb{N}$, (cf. (18)). This implies $f(s-z)^{-j} \in \mathcal{O}_{a, z}$ for $j=1, \cdots, k(z)$ and therefore also

$$
F^{z}=f \sum_{j=1}^{k(z)} P^{z, j}(s-z)^{-j} \in \mathcal{O}_{a, p}^{\ell \times \ell} .
$$


From (62) and (66) we also obtain

$$
\begin{gathered}
F^{z}=f^{z} \sum_{j=1}^{k(z)} P^{z, j}(s-z)^{k(z)-j} \text { and } \forall z^{\prime} \in V, z \neq z^{\prime}: \\
F^{z}=\left(s-z^{\prime}\right)^{k\left(z^{\prime}\right)} f^{z, z^{\prime}} \sum_{j=1}^{k(z)} P^{z, j}(s-z)^{k(z)-j} \in \\
\left(\mathcal{E}^{\prime} \bigcap \mathcal{O}\left(s-z^{\prime}\right)^{\left.k\left(z^{\prime}\right)\right)^{\ell \times \ell}}=\left(\mathcal{E}^{\prime}\left(s-z^{\prime}\right)^{k\left(z^{\prime}\right)}\right)^{\ell \times \ell} \Longrightarrow\right. \\
\forall z^{\prime} \neq z \text { and } w \in \mathcal{E}^{\ell} \text { with }\left(s-z^{\prime}\right)^{k\left(z^{\prime}\right)} \circ w=0: F^{z} \circ w=0 .
\end{gathered}
$$

Let

$$
\begin{gathered}
w \in\left(\mathcal{B}_{f} \bigcap \mathcal{P E}(-i z)\right)^{\ell}=\operatorname{ann}_{\mathcal{E}}\left((s-z)^{k(z)}\right)^{\ell}=\mathbb{C}[t]_{<k(z)}^{\ell} e^{-i z t} \Longrightarrow \\
\epsilon_{z} \circ w \underset{(69)}{=} w \text { and } R^{z} \circ w=\epsilon_{z} R^{z} \circ w=\left(F^{z}+f Q^{z}\right) \circ w_{f \circ w=0}^{=} F^{z} \circ w .
\end{gathered}
$$

In the representation of $\mathcal{B} \cap \mathcal{P E}(-i z)^{\ell}$ we have thus replaced $R^{z} \in \mathbb{C}[s]^{\ell \times \ell}$ by $F^{z} \in$ $\mathcal{O}_{a, p}^{\ell \times \ell}$.

For the final step we notice that if $\left(x^{z}\right)_{z \in V}, V$ countable, is any countable family of vectors $x^{z}$ in a Banach space $X$ with the norm $\|x\|$ one can choose positive real numbers $a(z)>0$ such that $\sum_{z \in V} a(z)\left\|x^{z}\right\|<\infty$. The completeness of $X$ then implies that $\sum_{z \in V} a(z) x^{z} \in X$. We apply this to the Banach space $\mathcal{O}_{a, p}^{\ell \times \ell}$ with the maximum norm $\|-\|_{a, p}$ induced from $\|-\|_{a, p}$ on $\mathcal{O}_{a, p}$ (cf. (18)), i.e.,

$$
Y \in \mathcal{O}_{a, p}^{\ell \times \ell}, Y_{j, k} \in \mathcal{O}_{a, p},\|Y\|_{a, p}=\max _{1 \leq j, k \leq \ell}\left\|Y_{j, k}\right\|_{a, p} .
$$

We choose positive real numbers $a(z)>0, z \in V$, such that $\sum_{z^{\prime} \in V} a\left(z^{\prime}\right)\left\|F^{z^{\prime}}\right\|_{a, p}<$ $\infty$ and obtain the convergent sum $R:=\sum_{z^{\prime} \in V} a\left(z^{\prime}\right) F^{z^{\prime}} \in \mathcal{O}_{a, p} \subset \mathcal{E}^{\prime}$. According to (20) the sum $R:=\sum_{z^{\prime} \in V} a\left(z^{\prime}\right) F^{z^{\prime}}$ is also convergent in the strong topology of $\mathcal{E}^{\prime}$ and therefore

$$
\forall w \in \mathcal{E}^{\ell}: R \circ w=\sum_{z^{\prime} \in V} a\left(z^{\prime}\right)\left(F^{z^{\prime}} \circ w\right)
$$

converges in the topology of $\mathcal{E}$. For $w \in \mathbb{C}[t]_{<k(z)} e^{-i z t}=\operatorname{ann}_{\mathcal{E}}\left((s-z)^{k(z)}\right)$ the preceding equations imply

$$
\begin{gathered}
R \circ w \underset{(85)}{=} \sum_{z^{\prime} \in V} a\left(z^{\prime}\right)\left(F^{z^{\prime}} \circ w\right) \underset{(82)}{=} a(z)\left(F^{z} \circ w\right) \underset{(83)}{=} a(z)\left(R^{z} \circ w\right) \Longrightarrow \\
\mathcal{B} \bigcap \mathcal{P E}(-i z)^{\ell}=\left\{w \in \mathbb{C}[t]_{<k(z)}^{\ell} e^{-i z t} ; R^{z} \circ w=0\right\}= \\
\left\{w \in \mathbb{C}[t]_{<k(z)}^{\ell} e^{-i z t} ; F^{z} \circ w=0\right\}=\left\{w \in \mathbb{C}[t]_{<k(z)}^{\ell} e^{-i t z} ; R \circ w=0\right\} .
\end{gathered}
$$

In the representation of $\mathcal{B} \cap \mathcal{P E}(-i z)^{\ell}$ we have thus replaced the various polynomial 
matrices $R^{z}$ by one matrix $R \in \mathcal{O}_{a, p}^{\ell \times \ell}$. We finally define

$$
\begin{aligned}
& \mathcal{B}_{1}:=\left(\mathcal{E}^{\prime 1 \times 2 \ell}\left(\underset{f \text { id }_{\ell}}{R}\right)\right)^{\perp}=\left\{w \in \mathcal{B}_{f}^{\ell} ; R \circ w=0\right\} \Longrightarrow f \circ \mathcal{B}_{1}=0 \text { and } \\
& \mathcal{B}_{1} \bigcap \mathcal{P E}(-i z)^{\ell}=\left\{w \in \mathbb{C}[t]_{<k(z)} e^{-i z t} ; R \circ w=0\right\}= \\
& \left\{w \in \mathbb{C}[t]_{<k(z)} e^{-i z t} ; R^{z} \circ w=0\right\}=\mathcal{B} \bigcap \mathcal{P E}(-i z)^{\ell} \Longrightarrow \\
& \mathcal{B}_{1} \bigcap \mathcal{P} \mathcal{E}^{\ell}=\bigoplus_{z \in V}\left(\mathcal{B}_{1} \bigcap \mathcal{P} \mathcal{E}(-i z)^{\ell}\right)=\bigoplus_{z \in V}\left(\mathcal{B} \bigcap \mathcal{P} \mathcal{E}(-i z)^{\ell}\right)=\mathcal{B} \bigcap \mathcal{P} \mathcal{E}^{\ell} \Longrightarrow \\
& \mathcal{B}=\operatorname{cl}_{E}\left(\mathcal{B} \bigcap \mathcal{P \mathcal { E } ^ { \ell }}\right)=\mathcal{B}_{1}=\left(\mathcal{E}^{\prime 1 \times 2 \ell}\left(\underset{f \text { id }_{\ell}}{R}\right)\right)^{\perp} \text {. }
\end{aligned}
$$

Corollary 5.9. ([30, Thm. 13 on p.914 ]) If $\mathfrak{a}$ is a nonzero closed ideal of $\mathcal{E}^{\prime}$ with gen. beh. $\mathcal{B}=\mathfrak{a}^{\perp} \subsetneq \mathcal{E}$ and $0 \neq f \in \mathfrak{a} \bigcap \mathcal{O}_{a, p}$ then there is $R \in \mathfrak{a} \bigcap \mathcal{O}_{a, p}$ such that

$$
\mathcal{B}=\{w \in \mathcal{E} ; R \circ w=f \circ w=0\} \text { and } \mathfrak{a}=\operatorname{cl}_{E}\left(\mathcal{E}^{\prime} f+\mathcal{E}^{\prime} R\right) \text {. }
$$

Proof. One applies Thm. 5.8 to the autonomous gen.beh. $\mathcal{B}$. Schwartz constructed $R$ differently and gave only an indication of the proof.

Corollary 5.10. If $\mathcal{B}_{1} \subseteq \mathcal{E}^{\ell_{1}}$ is an autonomous behavior and $P \in \mathcal{E}^{\ell_{2} \times \ell_{1}}$ then the closed image $\mathcal{B}_{2}:=\operatorname{cl}_{E}\left(P \circ \mathcal{B}_{1}\right)(c f$. (36)) is also autonomous and therefore a behavior.

The emphasis of [33] is on delay-differential behaviors. To discuss these we need the algebras

$$
\begin{array}{cccccc}
\mathcal{R}:= & \bigoplus_{h \in \mathbb{R}} \mathbb{C}\left[\delta^{\prime}\right] \delta_{h} & \subset & \mathcal{E}^{\prime} & \subset & \mathcal{O} \\
\mathcal{H}_{\mathcal{R}}:=\operatorname{quot}(\mathcal{R}) \cap \mathcal{O} & \subset & \mathcal{H}_{E}:=\mathcal{K} \cap \mathcal{O} & \subset & \bigcap_{\mathcal{O}}
\end{array}, \quad \mathcal{H}_{\mathcal{R}} \subset \mathcal{E}^{\prime},
$$

where quot $(A)$ denotes the quotient field of a domain $A, \mathcal{M}$ is the field of meromorphic functions on $\mathbb{C}$ and $\mathcal{R}$ is the ring of delay-differential operators. All nonzero distributions in $\mathcal{R}$ are invertible (cf. (22)) [9, p.697]. Therefore $\mathcal{H}_{\mathcal{R}}$ is contained in $\mathcal{E}^{\prime}$ and all nonzero distributions in $\mathcal{H}_{\mathcal{R}}$ are also invertible. Each $h \in \mathcal{H}_{\mathcal{R}}$ has the simpler form $h=f g^{-1}$ with $f \in \mathcal{R}$ and $0 \neq g \in \mathbb{C}[s]$ (cf. [17, Thm. 2.2], [5, (85)]). A behavior of the form $\mathcal{B}=\left\{w \in \mathcal{E}^{\ell} ; R \circ w=0\right\}$ with $R \in \mathcal{H}_{\mathcal{R}}^{k \times \ell}$ is called delay-differential [17, Def. 3.1]. The necessity of $\mathcal{H}_{\mathcal{R}}$ instead of $\mathcal{R}$ is explained in [15], [18] and [17].

Corollary 5.11. If a nonzero $f \in \mathcal{H}_{\mathcal{R}}$ annihilates a gen. beh. $\mathcal{B}$ this is autonomous and hence a behavior, but not delay-differential in general. The closed image of an autonomous delay-differential behavior is a behavior, but again not delay-differential in general.

Proof. Recall from Thm. 5.8 that

$$
\begin{aligned}
\mathcal{B} & =\left\{w \in \mathcal{E}^{\ell} ; R \circ w=0, f \circ w=0\right\} \text { with } \\
R & =\sum_{z \in V} a(z) F^{z}, F^{z}:=f \sum_{j=1}^{k(z)} P^{z, j}(s-z)^{-j} .
\end{aligned}
$$


If $f \in \mathcal{H}_{\mathcal{R}}$ then also all $(s-z)^{-j} f, 1 \leq j \leq k(z)$, belong to $\mathcal{H}_{\mathcal{R}}$ and therefore $F^{z} \in \mathcal{H}_{\mathcal{R}}^{\ell \times \ell}$ for all $z \in V$. But the infinite convergent sum $R=\sum_{z \in V} a(z) F^{z}$ will only rarely belong to $\mathcal{H}_{\mathcal{R}}^{\ell \times \ell}$. So $\mathcal{B}$ is, in general, not a delay-differential behavior.

\section{Characteristic variety and weak controllability}

Recall the algebras and especially the quotient fields from (50) and (89). Consider a f.g. $\mathcal{E}^{\prime}$-module $M=\mathcal{E}^{\prime 1 \times \ell} / U$ with the associated gen. beh. $\mathcal{B}:=U^{\perp}=\operatorname{cl}_{E}(U)^{\perp}$. From (54)- (57) we get

$$
\begin{aligned}
\mathcal{K} \otimes_{\mathcal{E}^{\prime}}\left(\operatorname{cl}_{E}(U) / U\right)= & \left(\mathcal{K} \otimes_{\mathcal{E}^{\prime}} \operatorname{cl}_{E}(U)\right) /\left(\mathcal{K} \otimes_{\mathcal{E}^{\prime}} U\right)=\mathcal{K} \operatorname{cl}_{E}(U) / \mathcal{K} U=0 \Longrightarrow \\
& \operatorname{cl}_{E}(U) / U \subseteq \operatorname{tor}(M)=U_{2} / U .
\end{aligned}
$$

Recall that the dimension $\operatorname{dim}_{A}(N)$ of a free module over a commutative ring is the length of one and then of all its bases. If $N$ is a f.g. module over a noetherian local integral domain $A$ with maximal ideal $\mathfrak{m}$, residue field $k=A / \mathfrak{m}$ and quotient field $K:=\operatorname{quot}(A)$ then $N / \mathfrak{m} N$ is a $k$-space and Krull's (Nakayama's) lemma shows that

$$
\begin{gathered}
\operatorname{dim}_{k}(N / \mathfrak{m} N) \geq \operatorname{rank}(N):=\operatorname{dim}_{K}\left(K \otimes_{A} N\right) \text { and } \\
\operatorname{dim}_{k}(N / \mathfrak{m} N)=\operatorname{rank}(N) \Longleftrightarrow N \text { is free of } \operatorname{dim}_{A}(N)=\operatorname{rank}(N) .
\end{gathered}
$$

For $z \in \mathbb{C}$, the DVR $A:=\mathcal{E}_{\mathfrak{m}_{E}(z)}^{\prime}$ with $\mathcal{E}_{\mathfrak{m}_{E}(z)}^{\prime} / \mathfrak{m}_{E}(z) \mathcal{E}_{\mathfrak{m}_{E}(z)}^{\prime}=\mathbb{C}$ and the f.g. $\mathcal{E}_{\mathfrak{m}(z)}^{\prime}$-module $M_{\mathfrak{m}_{E}(z)}$ with $M / \mathfrak{m}_{E}(z) M \underset{\text { ident. }}{=} M_{\mathfrak{m}_{E}(z)} / \mathfrak{m}_{E}(z) M_{\mathfrak{m}_{E}(z)}$ and $\operatorname{rank}(M)=$ $\operatorname{rank}\left(M_{\mathfrak{m}_{E}(z)}\right)$ this implies

$$
\begin{aligned}
\operatorname{dim}_{\mathbb{C}}\left(M / \mathfrak{m}_{E}(z) M\right) \geq m:=\operatorname{rank}(M)=\operatorname{rank}\left(M_{\mathfrak{m}_{E}(z)}\right) \text { and } \\
\operatorname{dim}_{\mathbb{C}}\left(M / \mathfrak{m}_{E}(z) M\right)=m \Longleftrightarrow M_{\mathfrak{m}_{E}(z)} \text { is free of dimension } m .
\end{aligned}
$$

Recall that the module $M_{\mathfrak{m}_{E}(z)}$ over the DVR $\mathcal{E}_{\mathfrak{m}_{E}(z)}^{\prime}$ is free if and only if it is torsionfree. If $U=\mathcal{E}^{\prime 1 \times k} R, R \in \mathcal{E}^{\prime k \times \ell}$ is itself f.g. and $\mathcal{B}=\left\{w \in \mathcal{E}^{\ell} ; R \circ w=0\right\}$ is a behavior then

$$
\begin{gathered}
p:=\operatorname{rank}(U)=\operatorname{rank}(R), m:=\operatorname{rank}(M)=\ell-p, \\
M / \mathfrak{m}_{E}(z) M \underset{\text { ident. }}{=} \mathbb{C}^{1 \times \ell} / \mathbb{C}^{1 \times k} R(z), \operatorname{dim}_{\mathbb{C}}\left(M / \mathfrak{m}_{E}(z) M\right)=\ell-\operatorname{rank}(R(z)) .
\end{gathered}
$$

We define the characteristic variety

$$
\begin{gathered}
\operatorname{char}(M)=\operatorname{char}(\mathcal{B}):=\left\{z \in \mathbb{C} ; M_{\mathfrak{m}_{E}(z)} \text { is not free }\right\}, \text { especially } \\
\operatorname{char}(M)=\operatorname{char}(\mathcal{B})=\{z \in \mathbb{C} ; \operatorname{rank}(R(z))<\operatorname{rank}(R)\} \text { in the situation of (93). }
\end{gathered}
$$

For all $z \in \mathbb{C} \backslash \operatorname{char}(M)$ the module $M_{\mathfrak{m}_{E}(z)}$ is free of dimension $\operatorname{rank}(M)$.

Recall that $\mathcal{P} \mathcal{E}(-i z)$ is an injective cogenerator over $\mathcal{E}_{\mathfrak{m}_{E}(z)}^{\prime}$ and

$$
\begin{gathered}
\mathcal{B} \bigcap \mathcal{P E}(-i z)^{\ell} \cong \operatorname{Hom}_{\mathcal{E}_{\mathfrak{m}_{E}(z)}^{\prime}}\left(M_{\mathfrak{m}_{E}(z)}, \mathcal{P} \mathcal{E}(-i z)\right), \text { hence } \\
\mathcal{B} \bigcap \mathcal{P} \mathcal{E}(-i z)^{\ell} \neq 0 \Longleftrightarrow M_{\mathfrak{m}_{E}(z)} \neq 0 .
\end{gathered}
$$


If $\mathcal{B}$ is autonomous and thus $M$ and all $M_{\mathfrak{m}_{E}(z)}$ are torsion modules then

$$
\begin{gathered}
\operatorname{char}(M)=\operatorname{char}(\mathcal{B})=\left\{z \in \mathbb{C} ; M_{\mathfrak{m}_{E}(z)} \neq 0\right\}=\left\{z \in \mathbb{C} ; \mathcal{B} \bigcap \mathcal{P E}(-i z)^{\ell} \neq 0\right\} \\
\text { and } \mathcal{B} \bigcap \mathcal{P} \mathcal{E}^{\ell}=\bigoplus_{z \in \operatorname{char}(\mathcal{B})}\left(\mathcal{B} \bigcap \mathcal{P} \mathcal{E}(-i z)^{\ell}\right) .
\end{gathered}
$$

The direct sum decomposition in (96) generalizes the well-known and important modal decomposition of one-dimensional autonomous differential behaviors.

The geometric properties of $\operatorname{char}(M)$ are further explained by studying the associated Stein modules $[5, \S 6]$. We define

$$
\begin{gathered}
\widehat{U}:=\operatorname{cl}_{O}(U) \underset{(53)}{=} \operatorname{cl}_{O}(\mathcal{O} U) \subseteq \mathcal{O}^{1 \times \ell} \text { and } \widehat{M}=\mathcal{O}^{1 \times \ell} / \widehat{U}, \text { hence (cf. (23), [5, Result 6.1] } \\
\widehat{U}_{z}=\mathcal{O}_{z} U=\mathcal{O}_{z} U_{\mathfrak{m}_{E}(z)}, \widehat{M}_{z}=\mathcal{O}_{z}^{1 \times \ell} / \widehat{U}_{z}=\mathcal{O}_{z} \otimes_{\mathcal{E}_{\mathfrak{m}_{E}(z)}^{\prime}} M_{\mathfrak{m}_{E}(z)}
\end{gathered}
$$

The submodule $\widehat{U}$ is closed and thus f.g. and free since $\mathcal{O}$ is a Bézout domain. Since the inclusion $\mathcal{E}_{\mathfrak{m}_{E}(z)}^{\prime} \subset \mathcal{O}_{z}$ is faithfully flat (cf. (31)) the module $M_{\mathfrak{m}_{E}(z)}$ is free if and only if $\widehat{M}_{z}$ is. The following properties are quoted from [5, $\S 8$, Result 8.1]. The characteristic variety $\operatorname{char}(\widehat{M})$ of $\widehat{M}[5,(108)]$ consists of the $z \in \mathbb{C}$ with nonfree $\widehat{M}_{z}$ and is indeed a variety. This implies

$$
\operatorname{char}(M)=\operatorname{char}(\widehat{M})=V_{\mathbb{C}}(g):=\{z \in \mathbb{C} ; g(z)=0\}
$$

for some entire function $g$ that does not necessarily belong to $\mathcal{E}^{\prime}$.

If $\operatorname{cl}_{E}(U)=\mathcal{E}^{\prime 1 \times \ell} \bigcap \operatorname{cl}_{O}(U) \subsetneq \mathcal{E}^{\prime 1 \times \ell}$ or, equivalently, $\widehat{M} \neq 0$ then $\operatorname{char}(\widehat{M}) \subsetneq \mathbb{C}$ and hence $g \neq 0$, $\operatorname{char}(M)$ is a countable discrete subset of $\mathbb{C}$ and $M_{\mathfrak{m}_{E}(z)}$ is free for almost all $z \in \mathbb{C}$. If $\operatorname{cl}_{E}(U)=\mathcal{E}^{\prime 1 \times \ell}$ then $M_{\mathfrak{m}_{E}(z)}=0$ for all $z \in \mathbb{C}$. The module $\widehat{M}$ is free if and only if $\operatorname{char}(\widehat{M})=\operatorname{char}(M)=\emptyset$. This also signifies that all $M_{\mathfrak{m}_{E}(z)}$ are free or torsionfree or that $\operatorname{dim}_{\mathbb{C}}\left(M / \mathfrak{m}_{E}(z) M\right)=\operatorname{rank}(M)$ for all $z \in \mathbb{C}$.

The next theorem characterizes weak controllability of a gen. beh. in the sense of [17, p. 11]). From (90) we derive that $U$ is closed if $M$ is torsionfree and therefore we assume the closedness of $U$ in the next theorem. We use the data from (54)- (57).

Theorem and Definition 6.1. (cf. [17, Thm. 3.12]) 1. If $U$ is closed the following properties are equivalent:

(i) $U=\mathcal{E}^{1 \times \ell} \bigcap \mathcal{K} U$.

(ii) $M \subset \mathcal{K} \otimes_{\mathcal{E}^{\prime}} M$, i.e., $M$ is torsionfree.

(iii) There is a matrix $P \in \mathcal{E}^{\prime \ell \times m}$ such that $\operatorname{ker}(\circ P)=\left\{\xi \in \mathcal{E}^{\prime 1 \times \ell} ; \xi P=0\right\}=U$ or $\left(\circ P_{\text {ind }}\right): M=\mathcal{E}^{1 \times \ell} / U \rightarrow \mathcal{E}^{\prime 1 \times m}$ is injective.

(iv) There is $P \in \mathcal{E}^{\prime \ell \times m}$ such that $\operatorname{cl}_{E}\left(P \circ \mathcal{E}^{m}\right)=\mathcal{B}$ or $P \circ \mathcal{P} \mathcal{E}^{m}=\mathcal{B} \cap \mathcal{P} \mathcal{E}^{\ell}$.

(v) $\operatorname{char}(\mathcal{B})=0$.

(vi) $\mathcal{B}=\operatorname{cl}_{E}\left(\mathcal{B} \cap \mathcal{D}^{\ell}\right)$.

Under these conditions $\mathcal{B}$ is called weakly controllable. The condition ( $v)$ is usually described as spectral controllability of $\mathcal{B}$.

(2) The gen. beh. $\mathcal{B}_{\text {cont }}:=\operatorname{Hom}_{\mathcal{E}^{\prime}}(M / \operatorname{tor}(M), \mathcal{E})=\operatorname{cl}_{E}\left(\mathcal{B} \cap \mathcal{D}^{\ell}\right)$ is the largest weakly controllable gen. beh. contained in $\mathcal{B}$. 
Proof. 1. $(i) \Longleftrightarrow(i i)$ :

$\operatorname{tor}(M)=\operatorname{ker}\left(\operatorname{can}: M=\mathcal{E}^{\prime 1 \times \ell} / U \rightarrow \mathcal{K} \otimes_{\mathcal{E}^{\prime}} M=\mathcal{K}^{1 \times \ell} / \mathcal{K} U\right)=\left(\mathcal{E}^{\prime 1 \times \ell} \bigcap \mathcal{K} U\right) / U$.

(ii) $\Longleftrightarrow($ iii): With the data from (57) we have

$\operatorname{tor}(M)=U_{2} / U=\operatorname{ker}\left((\circ P)_{\text {ind }}: \mathcal{E}^{\prime 1 \times \ell} / U \rightarrow \mathcal{E}^{\prime 1 \times m}\right), U_{2}=(\circ P)^{-1}(0)$.

(iii) $\Longleftrightarrow(i v)$ : Let $P \in \mathcal{E}^{\prime \ell \times m}$. Then

$$
U=(\circ P)^{-1}(0) \underset{\text { Cor. 4.7,(1) }}{\Longleftrightarrow} \operatorname{cl}_{E}\left(P \circ \mathcal{E}^{m}\right)=U^{\perp}=\mathcal{B} .
$$

$(i i) \Longrightarrow(v)$ : If $M$ is torsionfree then so are all $M_{\mathfrak{m}_{E}(z)}$.

$(v) \Longrightarrow(i i)$ : From $\operatorname{char}(M)=\emptyset$ we infer that all $M_{\mathfrak{m}_{E}(z)}$ are torsionfree or, equivalently, free. The equation (52) and $U=\mathrm{cl}_{E}(U)$ imply that the canonical map

$$
M=\mathcal{E}^{\prime 1 \times \ell} / U \rightarrow \prod_{z \in \mathbb{C}} M_{\mathfrak{m}_{E}(z)}=\prod_{z \in \mathbb{C}} \mathcal{E}_{\mathfrak{m}_{E}(z)}^{\prime \times \ell} / U_{\mathfrak{m}_{E}(z)}, \xi+U \mapsto\left(\xi+U_{\mathfrak{m}_{E}(z)}\right)_{z \in \mathbb{C}}
$$

is injective. Since all $M_{\mathfrak{m}_{E}(z)}$ are torsionfree over $\mathcal{E}_{\mathfrak{m}_{E}(z)}^{\prime}$ and hence over $\mathcal{E}^{\prime}$ also $M$ is torsionfree over $\mathcal{E}^{\prime}$.

$(i v) \Longrightarrow(v i)$ : Assume $\mathrm{cl}_{E}\left(P \circ \mathcal{E}^{m}\right)=\mathcal{B}$. Since $\mathcal{D}$ is dense in $\mathcal{E}$ we conclude

$$
\begin{gathered}
P \circ \mathcal{E}^{m}=P \circ \operatorname{cl}_{E}\left(\mathcal{D}^{m}\right) \subseteq \operatorname{cl}_{E}\left(P \circ \mathcal{D}^{m}\right) \subseteq \operatorname{cl}_{E}\left(\mathcal{B} \bigcap \mathcal{D}^{\ell}\right) \subseteq \mathcal{B} \Longrightarrow \\
\mathcal{B}=\operatorname{cl}_{E}\left(P \circ \mathcal{E}^{m}\right)=\operatorname{cl}_{E}\left(\mathcal{B} \bigcap \mathcal{D}^{\ell}\right)=\mathcal{B} .
\end{gathered}
$$

$(v i) \Longrightarrow(i i)$ : We proceed by contradiction. Assume that $M$ contains a nonzero torsion element or, in other words, that there is an injection $(\circ Q)_{\text {ind }}: \mathcal{E}^{\prime} / \mathfrak{a} \rightarrow M=\mathcal{E}^{\prime 1 \times \ell} / U$ with $Q \in \mathcal{E}^{\prime 1 \times \ell}$ and $0 \subsetneq \mathfrak{a} \subsetneq \mathcal{E}^{\prime}$. Since $(\circ Q)_{\text {ind }}$ is injective $\mathfrak{a}=(\circ Q)^{-1}(U)$ is the inverse image of $U$ under $\circ Q$ and thus closed. With (46) we conclude $\operatorname{cl}_{E}(Q \circ \mathcal{B})=$ $\mathfrak{a}^{\perp}$. Since $\mathfrak{a}$ is nonzero the behavior $\mathfrak{a}^{\perp}$ is autonomous. From Thm. 5.1 we infer $\mathfrak{a}^{\perp} \bigcap \mathcal{E}_{+}=0$ and hence $\mathfrak{a}^{\perp} \bigcap \mathcal{D}=0$. But

$$
\begin{gathered}
\mathcal{B}=\operatorname{cl}_{E}\left(\mathcal{B} \bigcap \mathcal{D}^{\ell}\right) \Longrightarrow Q \circ \mathcal{B} \subseteq \operatorname{cl}_{E}\left(Q \circ\left(\mathcal{B} \bigcap \mathcal{D}^{\ell}\right)\right) \subseteq \\
\operatorname{cl}_{E}((Q \circ \mathcal{B}) \bigcap \mathcal{D}) \subseteq \operatorname{cl}_{E}\left(\mathfrak{a}^{\perp} \bigcap \mathcal{D}\right)=\operatorname{cl}_{E}(0)=0 \Longrightarrow \\
\mathfrak{a}^{\perp}=\operatorname{cl}_{E}(Q \circ \mathcal{B})=0 \Longrightarrow \mathfrak{a}=\operatorname{cl}_{E}(\mathfrak{a})=\mathfrak{a}^{\perp \perp}=0^{\perp}=\mathcal{E}^{\prime} .
\end{gathered}
$$

This contradicts $\mathfrak{a} \subsetneq \mathcal{E}^{\prime}$.

2. We use tor $(M)=U_{2} / U$ with $U_{2}=(\circ P)^{-1}(0)$ from (57) and conclude $M / \operatorname{tor}(M)=$ $\mathcal{E}^{\prime 1 \times \ell} / U_{2}$ and

$$
\mathcal{B}_{\text {cont }}:=U_{2}^{\perp} \underset{\text { ident. }}{=} \operatorname{Hom}_{\mathcal{E}^{\prime}}(M / \operatorname{tor}(M), \mathcal{E}) .
$$

Since $M / \operatorname{tor}(M)$ is the largest torsionfree factor module of $M$ the gen. beh. $\mathcal{B}_{\text {cont }}$ is the largest weakly controllable gen. subbehavior of $\mathcal{B}$. Let $\mathcal{B}_{1}=\mathrm{cl}_{E}\left(\mathcal{B} \cap \mathcal{D}^{\ell}\right)$, hence

$$
\mathcal{B} \bigcap \mathcal{D}^{\ell} \subseteq \mathcal{B}_{1} \bigcap \mathcal{D}^{\ell} \subseteq \mathcal{B}_{1} \Longrightarrow \operatorname{cl}_{E}\left(\mathcal{B}_{1} \bigcap \mathcal{D}^{\ell}\right)=\mathcal{B}_{1} \Longrightarrow
$$

$\mathcal{B}_{1}$ weakly controllable $\Longrightarrow \mathcal{B}_{1} \subseteq \mathcal{B}_{\text {cont }}$ and $\mathcal{B}_{\text {cont }}=\operatorname{cl}_{E}\left(\mathcal{B}_{\text {cont }} \bigcap \mathcal{D}^{\ell}\right) \subseteq$

$$
\operatorname{cl}_{E}\left(\mathcal{B} \bigcap \mathcal{D}^{\ell}\right)=\mathcal{B}_{1} \Longrightarrow \mathcal{B}_{\text {cont }}=\mathcal{B}_{1}=\operatorname{cl}_{E}\left(\mathcal{B} \bigcap \mathcal{D}^{\ell}\right)
$$




\section{Input/output structures}

In this section we introduce input/output (IO) structures and the associated transfer matrices of gen. beh. and use them to show that also many nonautonomous gen. beh. are indeed behaviors.

Let $U \subseteq \mathcal{E}^{\prime 1 \times \ell}$ and $\mathcal{B}:=U^{\perp}$. Recall $p=\operatorname{rank}(U)=\operatorname{dim}_{\mathcal{K}}(\mathcal{K} U), m=\operatorname{rank}(M)$ and $p+m=\ell$ from (55). In general there are different subsets $I \subseteq\{1, \cdots, \ell\}$ with $p$ elements such that the projection $\mathcal{K}^{1 \times \ell} \rightarrow \mathcal{K}^{1 \times I}$ induces an isomorphism $\mathcal{K} U \cong$ $\mathcal{K}^{1 \times I}$. Such an $I$ is called an $I O$ structure of $U, M$ or $\mathcal{B}$ and $\mathcal{B}$ with this structure is called an IO gen. beh.. After the usual permutation of the components of $\mathcal{E}^{\prime 1 \times \ell}$ we always assume $I=\{1, \cdots, p\}$.

Lemma and Definition 7.1. There is a unique matrix $H \in \mathcal{K}^{p \times m}$ such that $\mathcal{K} U=$ $\mathcal{K}^{1 \times p}\left(\mathrm{id}_{p},-H\right)$ that is called the transfer matrix of the IO behavior with its chosen IO structure.

Proof. By assumption the projection

$$
\circ\left(\begin{array}{c}
\mathrm{id}_{p} \\
0
\end{array}\right): \mathcal{K} U \subset \mathcal{K}^{1 \times(p+m)} \rightarrow \mathcal{K}^{1 \times p},(\xi, \eta) \rightarrow \xi,
$$

is an isomorphism. Its inverse has the form

$$
\begin{aligned}
& \circ(X,-H): \mathcal{K}^{1 \times p} \cong \mathcal{K} U \subset \mathcal{K}^{1 \times(p+m)},(X,-H) \in \mathcal{K}^{p \times(p+m)} \Longrightarrow \\
& \operatorname{id}_{p}=(X,-H)\left(\begin{array}{c}
\operatorname{id}_{p} \\
0
\end{array}\right)=X,(X,-H)=\left(\operatorname{id}_{p},-H\right) \text { and } \mathcal{K} U=\mathcal{K}^{1 \times p}\left(\operatorname{id}_{p},-H\right) \text {. }
\end{aligned}
$$

Lemma 7.2 below shows that for behaviors instead of gen. beh. this transfer matrix coincides with the usual one that is known from differential IO behaviors and was also used in [17, Thm. 3.9]. Equation (54) implies that IO structures and transfer matrices of $U, \operatorname{cl}_{E}(U)$ and $U_{\mathfrak{m}_{E}(z)}$ coincide. An IO structure gives rise to the isomorphism

$$
\left(\circ\left(0, \mathrm{id}_{m}\right)\right)_{\text {ind }}: \mathcal{K}^{1 \times m} \rightarrow \mathcal{K} \otimes_{\mathcal{E}^{\prime}} M=\mathcal{K}^{1 \times(p+m)} / \mathcal{K} U, \eta \mapsto(0, \eta)+\mathcal{K} U,
$$

and then to the usual exact sequences

$$
\begin{aligned}
& 0 \rightarrow \mathcal{E}^{\prime 1 \times m} \stackrel{\left(\circ\left(0, \text {,id }_{m}\right)\right)_{\text {ind }}}{\longrightarrow} M \stackrel{\left.\left(\circ \stackrel{\left(\mathrm{id}_{p}\right)}{0}\right)\right)_{\text {ind }}}{\longrightarrow} M^{0} \rightarrow 0 \text { where } \\
& M=\mathcal{E}^{\prime 1 \times(p+m)} / U, M^{0}:=\mathcal{E}^{\prime 1 \times p} / U^{0}, U^{0}:=U\left(\begin{array}{c}
\operatorname{id}_{p} \\
0
\end{array}\right), \\
& \left.\left.0 \rightarrow \mathcal{E}_{\mathfrak{m}_{E}(z)}^{\prime 1 \times m} \stackrel{\left(\circ\left(0, \text {,id }_{m}\right)\right.}{\longrightarrow}\right)_{\text {ind }} M_{\mathfrak{m}_{E}(z)} \stackrel{\left(\circ\left(\begin{array}{c}
\mathrm{id}_{p} \\
0
\end{array}\right)\right.}{\longrightarrow}\right)_{\text {ind }} M_{\mathfrak{m}_{E}(z)}^{0} \rightarrow 0, z \in \mathbb{C} .
\end{aligned}
$$

The isomorphism (100) implies $\mathcal{K} \otimes_{\mathcal{E}^{\prime}} M^{0}=0$, hence $M^{0}$ is a torsion module and thus $\mathcal{B}^{0}:=\left(U^{0}\right)^{\perp} \subset \mathcal{E}^{p}$ is autonomous. According to Thm. 5.8 there is a matrix

$$
\begin{gathered}
P \in \mathcal{E}^{\prime k \times p}, \operatorname{rank}(P)=p, k=2 p, \text { with } \operatorname{cl}_{E}\left(U^{0}\right)=\operatorname{cl}_{E}\left(\mathcal{E}^{\prime 1 \times k} P\right), \text { hence } \\
U_{\mathfrak{m}_{E}(z)}^{0}= \begin{cases}\mathcal{E}_{\mathfrak{m}_{E}(z)}^{\prime 1 \times k} P & \text { if } z \in \operatorname{char}\left(M^{0}\right) \\
\mathcal{E}_{\mathfrak{m}_{E}(z)}^{\prime 1 \times p} & \text { if } z \in \mathbb{C} \backslash \operatorname{char}\left(M^{0}\right)\end{cases} \\
M_{\mathfrak{m}_{E}(z)}^{0}=\left\{\begin{array}{ll}
\mathcal{E}_{\mathfrak{m}_{E}(z)}^{1 \times p} / \mathcal{E}_{\mathfrak{m}_{E}(z)}^{1 \times k} P & \text { if } z \in \operatorname{char}\left(M^{0}\right) \\
0 & \text { if } z \in \mathbb{C} \backslash \operatorname{char}\left(M^{0}\right)
\end{array} .\right.
\end{gathered}
$$


Application of $\operatorname{Hom}_{\mathcal{E}^{\prime}}(-, \mathcal{E})$ resp. $\operatorname{Hom}_{\mathcal{E}^{\prime}}(-, \mathcal{P} \mathcal{E})$ to the first exact sequence in $(101)$ furnishes the exact gen. beh. sequence

$$
\begin{aligned}
& \begin{array}{ccccc}
0 \rightarrow \mathcal{B}^{0} & \stackrel{\text { inj }}{\longrightarrow} & \mathcal{B} & \stackrel{\text { proj }}{\longrightarrow} & \mathcal{E}^{m} \\
y & \mapsto & \left(\begin{array}{l}
y \\
0
\end{array}\right),\left(\begin{array}{l}
y \\
u
\end{array}\right) & \mapsto & u
\end{array} \text { and } \\
& \operatorname{cl}_{E}(\operatorname{proj}(\mathcal{B}))=\mathcal{E}^{m}, \operatorname{proj}\left(\mathcal{B} \bigcap \mathcal{P} \mathcal{E}^{p+m}\right)=\mathcal{P} \mathcal{E}^{m} \text {. }
\end{aligned}
$$

In particular, every polynomial-exponential input $u$ gives rise to a trajectory $(y, u)^{\top} \in$ $\mathcal{B}$. Even if $U$ is closed $U^{0}$ is not necessarily so, but $\operatorname{cl}_{E}(U)\left(\begin{array}{c}\operatorname{id}_{P} \\ 0\end{array}\right) \subseteq \operatorname{cl}_{E}\left(U\left(\begin{array}{c}\operatorname{id}_{P} \\ 0\end{array}\right)\right)$ implies

$$
\begin{gathered}
\operatorname{cl}_{E}\left(\mathcal{E}^{\prime 1 \times k} P\right)=\operatorname{cl}_{E}\left(U^{0}\right)=\operatorname{cl}_{E}\left(\operatorname{cl}_{E}(U)^{0}\right) \text { and } \\
\mathcal{B}^{0}=\left\{w \in \mathcal{E}^{p} ; P \circ w=0\right\}=\left(U^{0}\right)^{\perp}=\operatorname{cl}_{E}\left(U^{0}\right)^{\perp}=\left(\operatorname{cl}_{E}(U)^{0}\right)^{\perp} .
\end{gathered}
$$

In particular, $\mathcal{B}^{0}$ does not depend on the choice of $U$ with $U^{\perp}=\mathcal{B}$.

Lemma 7.2. If the IO gen. beh. $\mathcal{B}$ is a behavior, i.e., if there is a matrix

$$
\left(P_{1},-Q_{1}\right) \in \mathcal{E}^{\prime k_{1} \times(p+m)} \text { such that } \operatorname{cl}_{E}(U)=\operatorname{cl}_{E}\left(\mathcal{E}^{\prime \times k_{1}}\left(P_{1},-Q_{1}\right)\right)
$$

then as usual

$$
\begin{gathered}
\operatorname{rank}\left(P_{1},-Q_{1}\right)=\operatorname{rank}\left(P_{1}\right)=p, P_{1} H=Q_{1}, \\
\mathcal{B}=\left\{(y, u)^{\top} \in \mathcal{E}^{p+m} ; P_{1} \circ y=Q_{1} \circ u\right\}, \mathcal{B}^{0}=\left\{y \in \mathcal{E}^{p} ; P_{1} \circ y=0\right\} .
\end{gathered}
$$

Proof. For each $z \in \mathbb{C}$ we get

$$
\begin{gathered}
U_{\mathfrak{m}_{E}(z)}=\mathcal{E}_{\mathfrak{m}_{E}(z)}^{\prime \times k_{1}}\left(P_{1},-Q_{1}\right) \subset \mathcal{K} U_{\mathfrak{m}_{E}(z)}=\mathcal{K}^{1 \times k_{1}}\left(P_{1},-Q_{1}\right)=\mathcal{K}^{1 \times p}\left(\mathrm{id}_{p},-H\right) \Longrightarrow \\
p=\operatorname{rank}\left(P_{1},-Q_{1}\right) \text { and }\left(P_{1},-Q_{1}\right)=X\left(\operatorname{id}_{p},-H\right), X \in \mathcal{K}^{k_{1} \times p} \Longrightarrow \\
P_{1}=X, Q_{1}=X H=P_{1} H \Longrightarrow p=\operatorname{rank}\left(P_{1},-Q_{1}\right)=\operatorname{rank}\left(P_{1}\right) .
\end{gathered}
$$

Moreover

$$
\begin{gathered}
U^{0}=U\left(\begin{array}{c}
\operatorname{id}_{p} \\
0
\end{array}\right) \subseteq \operatorname{cl}_{E}\left(\mathcal{E}^{\prime 1 \times k_{1}}\left(P_{1},-Q_{1}\right)\right)\left(\begin{array}{c}
\operatorname{id}_{p} \\
0
\end{array}\right) \subseteq \operatorname{cl}_{E}\left(\mathcal{E}^{\prime 1 \times k_{1}}\left(P_{1},-Q_{1}\right)\left(\begin{array}{c}
\operatorname{id}_{p} \\
0
\end{array}\right)\right)= \\
\operatorname{cl}_{E}\left(\mathcal{E}^{\prime 1 \times k_{1}} P_{1}\right) \subseteq \operatorname{cl}_{E}\left(\operatorname{cl}_{E}(U)\left(\begin{array}{c}
\operatorname{id}_{p} \\
0
\end{array}\right)\right)=\operatorname{cl}_{E}\left(\operatorname{cl}_{E}(U)^{0}\right) \underset{(104)}{=} \operatorname{cl}_{E}\left(U^{0}\right) \Longrightarrow \\
\operatorname{cl}_{E}\left(U^{0}\right)=\operatorname{cl}_{E}\left(\mathcal{E}^{\prime 1 \times k_{1}} P_{1}\right), \mathcal{B}^{0}=\left\{w \in \mathcal{E}^{p} ; P_{1} \circ w=0\right\} .
\end{gathered}
$$

Notice that for an arbitrary matrix $H \in \mathcal{K}^{p \times m}$ there are various matrices

$$
\begin{gathered}
\left(P_{1},-Q_{1}\right) \in \mathcal{E}^{k_{1} \times(p+m)} \text { with } p=\operatorname{rank}\left(P_{1}\right) \text { and } P_{1} H=Q_{1} \text {. Then } \\
\mathcal{B}_{1}=\left\{(y, u)^{\top} \in \mathcal{E}^{p+m} ; P_{1} \circ y=Q_{1} \circ u\right\}
\end{gathered}
$$

is called a behavior realization of $H$.

For the algebra $\mathcal{H}_{E}:=\mathcal{K} \cap \mathcal{O}$ from (89) we obtain the representation

Lemma 7.3. $\mathcal{H}_{E}=\mathcal{K} \cap \mathcal{O}=\bigcap_{z \in \mathbb{C}} \mathcal{E}_{\mathfrak{m}_{E}(z)}^{\prime}$ 
Proof. Let $f g^{-1} \in \mathcal{K}=\operatorname{quot}\left(\mathcal{E}^{\prime}\right), f, g \in \mathcal{E}^{\prime}, g \neq 0$. Then

$$
\begin{aligned}
\forall z & \in \mathbb{C}: f g^{-1} \in \mathcal{E}_{\mathfrak{m}_{E}(z)}^{\prime} \Longleftrightarrow \forall z \in \mathbb{C}: \mathcal{E}^{\prime} f \subseteq \mathcal{E}_{\mathfrak{m}_{E}(z)}^{\prime} g=\left(\mathcal{E}^{\prime} g\right)_{\mathfrak{m}_{E}(z)} \Longleftrightarrow \\
\mathcal{E}^{\prime} f & \subseteq \mathcal{E}^{\prime} \bigcap \bigcap_{z \in \mathbb{C}}\left(\mathcal{E}^{\prime} g\right)_{\mathfrak{m}_{E}(z)} \underset{(52)}{\overline{(52}} \operatorname{cl}_{E}\left(\mathcal{E}^{\prime} g\right) \underset{(53)}{=} \mathcal{E}^{\prime} \bigcap \mathcal{O} g \Longleftrightarrow f g^{-1} \in \mathcal{O} .
\end{aligned}
$$

Compare the preceding proof with arguments in [17, p.4].

Lemma 7.4. The IO structures of the gen. beh. $\mathcal{B}$ and its weakly controllable subbehavior $\mathcal{B}_{\text {cont }}$ and the corresponding transfer matrices coincide.

Proof. Let $H$ be the transfer matrix of $\mathcal{B}$, i.e., $\mathcal{K} U=\mathcal{K}^{1 \times p}\left(\mathrm{id}_{p},-H\right)$. Recall from (57) and Thm. 6.1,(2), that

$$
\begin{gathered}
\operatorname{tor}(M)=U_{2} / U \subseteq M=\mathcal{E}^{\prime 1 \times \ell} / U, M / \operatorname{tor}(M)=\mathcal{E}^{\prime 1 \times \ell} / U_{2}, \\
\mathcal{B}_{\text {cont }}=U_{2}^{\perp} \subseteq \mathcal{B}=U^{\perp} .
\end{gathered}
$$

The equation $\mathcal{K} \otimes_{\mathcal{E}^{\prime}}$ tor $(M)=0$ and the application of the exact functor $\mathcal{K} \otimes_{\mathcal{E}^{\prime}}(-)$ to the exact sequence $0 \rightarrow \operatorname{tor}(M) \subset M \rightarrow M / \operatorname{tor}(M) \rightarrow 0$ then furnish

$$
\begin{gathered}
\mathcal{K} \otimes_{\mathcal{E}^{\prime}} M=\mathcal{K}^{1 \times \ell} / \mathcal{K} U \cong \mathcal{K} \otimes_{\mathcal{E}^{\prime}}(M / \operatorname{tor}(M))=\mathcal{K}^{1 \times \ell} / \mathcal{K} U_{2} \Longrightarrow \\
\mathcal{K} U=\mathcal{K} U_{2} \Longrightarrow \mathcal{K} U_{2}=\mathcal{K}^{1 \times p}\left(\operatorname{id}_{p},-H\right) .
\end{gathered}
$$

The next theorem shows that also various nonautonomous gen. beh. are indeed behaviors and thus extends Thm. 5.8.

Theorem 7.5. Assume an IO structure $\mathcal{K} U=\mathcal{K}^{1 \times p}\left(\operatorname{id}_{p},-H\right)$ of $\mathcal{B}$ and a matrix $P \in \mathcal{E}^{\prime k \times p}$ as in (102) and (104) and define the matrix $Q:=P H \in \mathcal{K}^{k \times m}$. Then

1. $Q \in \mathcal{H}_{E}^{k \times m}$ (cf. Lemma 7.3)

2. If $Q \in \mathcal{E}^{\prime k \times m}$ then $\mathcal{B}$ is a behavior, viz. $\mathcal{B}=\left\{\left(\begin{array}{l}y \\ u\end{array}\right) \in \mathcal{E}^{p+m} ; P \circ y=Q \circ u\right\}$. This is the case if $U^{0}$ is closed.

3. If $H$ has an invertible common denominator $f\left(0 \neq f \in \mathcal{E}^{\prime}, f H \in \mathcal{E}^{\prime p \times m}\right)$ then $Q \in \mathcal{E}^{\prime k \times m}$ and hence $\mathcal{B}$ is a behavior by item 2.. Moreover the controllable part $\mathcal{B}_{\text {cont }}$ of $\mathcal{B}$ is a behavior and the projection proj : $\mathcal{B} \rightarrow \mathcal{E}^{m},\left(\begin{array}{c}y \\ u\end{array}\right) \mapsto u$, is surjective (cf. [17, Thm. 3.9]).

Proof. 1. For every $z \in \mathbb{C}$ the IO structure is also one of $U_{\mathfrak{m}_{E}(z)}$. Since $\mathcal{E}_{\mathfrak{m}_{E}(z)}^{\prime}$ is a DVR the submodule $U_{\mathfrak{m}_{E}(z)}$ of $\operatorname{rank}\left(U_{\mathfrak{m}_{E}(z)}\right)=\operatorname{rank}(U)$ has the form

$$
\begin{gathered}
U_{\mathfrak{m}_{E}(z)}=\mathcal{E}_{\mathfrak{m}_{E}(z)}^{\prime 1 \times p}\left(P^{z},-Q^{z}\right),\left(P^{z},-Q^{z}\right) \in \mathcal{E}_{\mathfrak{m}_{E}(z)}^{p \times(p+m)}, P^{z} H=Q^{z} \Longrightarrow \\
\mathcal{E}_{\mathfrak{m}_{E}(z)}^{\prime 1 \times k} P=U_{\mathfrak{m}_{E}(z)}^{0}=U_{\mathfrak{m}_{E}(z)}\left(\begin{array}{c}
\operatorname{id}_{p} \\
0
\end{array}\right)=\mathcal{E}_{\mathfrak{m}_{E}(z)}^{\prime 1 \times p} P^{z} \Longrightarrow P=X^{z} P^{z}, X^{z} \in \mathcal{E}_{\mathfrak{m}_{E}(z)}^{\prime k \times p} \\
\Longrightarrow Q=P H=X^{z} P^{z} H=X^{z} Q^{z} \in \mathcal{E}_{\mathfrak{m}_{E}(z)}^{\prime k \times m} \Longrightarrow Q \in \bigcap_{z \in \mathbb{C}} \mathcal{E}_{\mathfrak{m}_{E}(z)}^{\prime k \times m}=\mathcal{H}_{E}^{k \times m} .
\end{gathered}
$$


2. Assume $Q \in \mathcal{E}^{\prime k \times m}$. By part 1 . of the proof we obtain

$$
\begin{gathered}
U_{\mathfrak{m}_{E}(z)}=\mathcal{E}_{\mathfrak{m}_{E}(z)}^{1 \times p}\left(P^{z},-Q^{z}\right)=\mathcal{E}_{\mathfrak{m}_{E}(z)}^{1 \times p} P^{z}\left(\operatorname{id}_{p},-H\right)= \\
\mathcal{E}_{\mathfrak{m}_{E}(z)}^{\prime 1 \times k} P\left(\operatorname{id}_{p},-H\right)=\mathcal{E}_{\mathfrak{m}_{E}(z)}^{\prime 1 \times k}(P,-Q) \underset{(52)}{\Longrightarrow} \operatorname{cl}_{E}(U)=\operatorname{cl}_{E}\left(\mathcal{E}^{\prime 1 \times k}(P,-Q)\right),
\end{gathered}
$$

hence $\mathcal{B}=U^{\perp}=\left\{\left(\begin{array}{c}y \\ u\end{array}\right) \in \mathcal{E}^{p+m} ; P \circ y=Q \circ u\right\}$ is indeed an IO behavior. If $U^{0}$ is closed then $\mathcal{E}^{\prime 1 \times k} P \subseteq U^{0}$ and hence

$$
\begin{gathered}
\mathcal{E}^{\prime 1 \times k}(P,-Q)=\mathcal{E}^{\prime 1 \times k} P\left(\mathrm{id}_{P},-H\right) \subseteq U^{0}\left(\mathrm{id}_{p},-H\right)=U \subseteq \mathcal{E}^{\prime \times(p+m)} \\
\Longrightarrow Q \in \mathcal{E}^{\prime k \times m} .
\end{gathered}
$$

3. Let $0 \neq f \in \mathcal{E}^{\prime}$ be an invertible common denominator of $H$, i.e.,

$$
\begin{gathered}
\mathcal{E}^{\prime} f=\mathcal{E}^{\prime} \bigcap \mathcal{O} f \text { or } \mathcal{E}^{\prime}=f^{-1} \mathcal{E}^{\prime} \bigcap \mathcal{O} \text { and } f H \in \mathcal{E}^{\prime p \times m} \Longrightarrow \\
f Q=P(f H) \in \mathcal{E}^{\prime k \times m} \text { and } Q=f^{-1}(f Q) \in f^{-1} \mathcal{E}^{\prime k \times m} \bigcap \mathcal{O}^{k \times m}=\mathcal{E}^{k \times m} .
\end{gathered}
$$

Part 2. and Lemma 7.4 imply that $\mathcal{B}$ and $\mathcal{B}_{\text {cont }}$ are behaviors. Finally consider an equation $P \circ y=Q \circ u$ with given input $u$. Since $f$ is invertible there is a

$$
\begin{gathered}
v \in \mathcal{E}^{m} \text { with } u=f \circ v \Longrightarrow \\
P \circ((f H) \circ v)=P f H \circ v=Q f \circ v=Q \circ(f \circ v)=Q \circ u \Longrightarrow \\
\mathcal{B} \rightarrow \mathcal{E}^{m},\left(\begin{array}{c}
y \\
u
\end{array}\right) \mapsto u, \text { is surjective. }
\end{gathered}
$$

Corollary 7.6. (cf. [32, Thm. 3.8], [17, Thm. 3.9, lines before Prop. 3.13]). If in Thm. 7.5 the transfer matrix $H$ belongs to quot $(\mathcal{R})(c f .(89))$ it has an invertible common denominator and therefore $\mathcal{B}$ and $\mathcal{B}_{\text {cont }}(c f$. Lemma 7.4) are IO behaviors. In particular, if $\mathcal{B}$ is a delay-differential behavior then $\mathcal{B}_{\text {cont }}$ is a behavior, but it is not known at present whether $\mathcal{B}_{\text {cont }}$ is delay-differential too.

Proof. All nonzero delay-differential operators $g \in \mathcal{R}$ are invertible and so item 3. of Thm. 7.5 is applicable.

We conjecture that the weakly controllable part of a delay-differential behavior is not necessarily a delay-differential behavior.

Lemma 7.7. (Rosenbrock equations) Let $\mathcal{B}_{1} \subseteq \mathcal{E}^{n+m}$ be an IO gen. beh. with transfer matrix $H_{1}$ and autonomous part $\mathcal{B}_{1}^{0}, P=\left(\begin{array}{cc}Y & U \\ 0 & \operatorname{id}_{m}\end{array}\right) \in \mathcal{E}^{\prime(p+m) \times(n+m)}$ and

$$
\mathcal{B}_{2}:=\operatorname{cl}_{E}\left(P \circ \mathcal{B}_{1}\right)=\operatorname{cl}_{E}\left\{\left(\begin{array}{c}
Y \circ x+U \circ u \\
u
\end{array}\right) ;\left(\begin{array}{l}
x \\
u
\end{array}\right) \in \mathcal{B}_{1}\right\} \subseteq \mathcal{E}^{p+m} .
$$

Then also $\mathcal{B}_{2}$ is an $I O$ gen. beh. with transfer matrix $H_{2}=U+Y H_{1}$ and autonomous part $\mathcal{B}_{2}^{0}=\operatorname{cl}_{E}\left(Y \circ \mathcal{B}_{1}^{0}\right)$.

Proof. Let $U_{1}:=\mathcal{B}_{1}^{\perp}, U_{1}^{0}:=U_{1}\left(\begin{array}{c}\operatorname{id}_{n} \\ 0\end{array}\right), \mathcal{K} U_{1}=\mathcal{K}^{1 \times n}\left(\mathrm{id}_{n},-H_{1}\right)$ and consider the map

$$
\begin{gathered}
\circ P=\circ\left(\begin{array}{cc}
Y & U \\
0 & \operatorname{id}_{m}
\end{array}\right): \mathcal{E}^{1 \times(p+m)} \rightarrow \mathcal{E}^{\prime 1 \times(n+m)},\left(\xi_{2}, \eta\right) \mapsto\left(\xi_{2} Y, \xi_{2} U+\eta\right), \Longrightarrow \\
U_{2}:=(\circ P)^{-1}\left(U_{1}\right) \text { closed }, \mathcal{B}_{2} \underset{(46)}{=} U_{2}^{\perp}, U_{2}^{0}=U_{2}\left(\begin{array}{c}
\operatorname{id}_{p} \\
0
\end{array}\right), \\
\mathcal{K} U_{2}:=(\circ P)^{-1}\left(\mathcal{K} U_{1}\right)=(\circ P)^{-1}\left(\mathcal{K}^{1 \times n}\left(\operatorname{id}_{n},-H_{1}\right)\right) .
\end{gathered}
$$


By two easy computations these equations furnish

$$
\mathcal{K} U_{2}=\mathcal{K}^{1 \times p}\left(\mathrm{id}_{p},-\left(U+Y H_{1}\right)\right) \text { and } U_{2}^{0}:=(\circ Y)^{-1}\left(U_{1}^{0}\right) .
$$

These equations signify that $\mathcal{B}_{2}=\operatorname{cl}_{E}\left(P \circ \mathcal{B}_{1}\right)$ is an IO gen. beh. with transfer matrix $H_{2}:=U+Y H_{1}$ and autonomous part $\mathcal{B}_{2}^{0} \underset{(46)}{=} \operatorname{cl}_{E}\left(Y \circ \mathcal{B}_{1}^{0}\right)$.

The connection with the standard Rosenbrock equations is the following: If in Lemma $7.7 \mathcal{B}_{1}$ is an IO behavior of the form

$$
\begin{gathered}
\mathcal{B}_{1}=\left\{\left(\begin{array}{l}
x \\
u
\end{array}\right) \in \mathcal{E}^{n+m} ; P_{1} \circ x=Q_{1} \circ u\right\} \text { then } \\
\mathcal{B}_{2}:=\operatorname{cl}_{E}\left\{\left(\begin{array}{l}
y \\
u
\end{array}\right) \in \mathcal{E}^{p+m} ; \exists x \in \mathcal{E}^{n}, u \in \mathcal{E}^{m}: P_{1} \circ x=Q_{1} \circ u, y=Y \circ x+U \circ u\right\}
\end{gathered}
$$

is an IO gen. beh. with transfer matrix $H_{2}=U+Y H_{1}$ where $P_{1} H_{1}=Q_{1}$. The difference to the case of differential systems is that the closure operator $\mathrm{cl}_{E}$ is needed and that it is generally not known whether $\mathcal{B}_{2}$ is a behavior. But we have

Theorem 7.8. If in Lemma 7.7 the transfer matrix $H_{1}$ of $\mathcal{B}_{1}$ has an invertible common denominator, for instance if $H_{1} \in q u o t(\mathcal{R})^{n \times m}$ or if $\mathcal{B}_{1}$ is a delay-differential behavior, then also $H_{2}$ has an invertible common denominator and therefore both $\mathcal{B}_{1}$ and $\mathcal{B}_{2}$ are behaviors.

Proof. A common denominator of $H_{1}$ is also one of $H_{2}=U+Y H_{1}$, hence the theorem follows from Thm. 7.5.

In Thm. 7.8 one obtains a behavior $\mathcal{B}_{2}$ by eliminating the latent variable $x$ from the behavior $\mathcal{B}_{1}$, but without any rank or observability condition, cf. [17, Thm. 3.7]. There remains the question whether all gen. beh. are indeed behaviors. The following considerations suggest that this is not the case, but that counter-examples are hard to construct. We use the important Thm. 2.12 from [32]. Result 8.1 from [5] (cf. (98)) shows that a Stein module $\widehat{C}=\mathcal{O}^{1 \times \ell} / \mathcal{O}^{1 \times k} R, R \in \mathcal{O}^{k \times \ell}$, is torsionfree and then indeed free of dimension $\ell-r, r:=\operatorname{rank}(R)$, if and only if $\operatorname{char}(\widehat{C})=\emptyset$ or, equivalently, $\operatorname{rank}(R(z))=\operatorname{rank}(R):=r$ for all $z \in \mathbb{C}$. This is also equivalent to the freeness of $\mathcal{O}^{k} / R \mathcal{O}^{\ell}$ or to the existence of a generalized inverse $G \in \mathcal{O}^{\ell \times k}$ of $R$ with $R G R=R[32$, Remark 2.10].

Result 7.9. ([32, Thm. 2.12]) Assume $R \in \mathcal{E}^{\prime k \times \ell}$ and $\operatorname{rank}(R(z))=\operatorname{rank}(R)$ and hence also $\operatorname{rank}\left(R(z)^{\top}\right)=\operatorname{rank}\left(R^{\top}\right)$ for all $z \in \mathbb{C}$. Then there are $k^{\prime}, \ell^{\prime \prime} \in \mathbb{N}$ and $R^{\prime} \in \mathcal{E}^{\prime k^{\prime} \times k}, R^{\prime \prime} \in \mathcal{E}^{\prime \ell \times \ell^{\prime \prime}}$ such that the sequences

$$
\mathcal{O}^{\prime 1 \times k^{\prime}} \stackrel{\circ R^{\prime}}{\longrightarrow} \mathcal{O}^{1 \times k} \stackrel{\circ R}{\longrightarrow} \mathcal{O}^{1 \times \ell} \stackrel{\circ R^{\prime \prime}}{\longrightarrow} \mathcal{O}^{1 \times \ell^{\prime \prime}} \text { and } \mathcal{O}^{\prime k^{\prime}} \stackrel{R^{\prime} \circ}{\longleftarrow} \mathcal{O}^{k} \stackrel{R \circ}{\longleftarrow} \mathcal{O}^{\ell} \stackrel{R^{\prime \prime} \circ}{\longleftarrow} \mathcal{O}^{\ell^{\prime \prime}}
$$

are exact and especially $\operatorname{rank}\left(R^{\prime}\right)+\operatorname{rank}(R)=k, \operatorname{rank}(R)+\operatorname{rank}\left(R^{\prime \prime}\right)=\ell$.

This result follows by application of [32, Thm. 2.12] to $R$ and to $R^{\top}$. For all $z \in \mathbb{C}$ the inclusion $\mathcal{E}_{\mathfrak{m}_{E}(z)}^{\prime} \subset \mathcal{O}_{z}$ is faithfully flat and a sequence $\mathfrak{M}$ of Stein modules is exact if and only if all sequences $\mathfrak{M}_{z}, z \in \mathbb{C}$, are exact [5, Result 6.1]. We conclude

Corollary 7.10. For the data from Result 7.9 and all $z \in \mathbb{C}$ the sequences

$$
\begin{aligned}
& \mathcal{E}_{\mathfrak{m}_{E}(z)}^{\prime 1 \times k^{\prime}} \stackrel{\circ R^{\prime}}{\longrightarrow} \mathcal{E}_{\mathfrak{m}_{E}(z)}^{\prime 1 \times k} \stackrel{\circ R}{\longrightarrow} \mathcal{E}_{\mathfrak{m}_{E}(z)}^{1 \times \ell} \stackrel{\circ R^{\prime \prime}}{\longrightarrow} \mathcal{E}_{\mathfrak{m}_{E}(z)}^{1 \times \ell^{\prime \prime}} \\
& \mathcal{E}_{\mathfrak{m}_{E}(z)}^{\prime k^{\prime}} \stackrel{R^{\prime} \circ}{\longleftarrow} \mathcal{E}_{\mathfrak{m}_{E}(z)}^{\prime k} \stackrel{R \circ}{\longleftarrow} \mathcal{E}_{\mathfrak{m}_{E}(z)}^{\prime \ell} \stackrel{R^{\prime \prime} \circ}{\longleftarrow} \mathcal{E}_{\mathfrak{m}_{E}(z)}^{1 \times \ell^{\prime \prime}}
\end{aligned}
$$


and therefore also the sequences

$$
\begin{gathered}
\mathcal{K}^{1 \times k^{\prime}} \stackrel{\circ R^{\prime}}{\longrightarrow} \mathcal{K}^{1 \times k} \stackrel{\circ R}{\longrightarrow} \mathcal{K}^{1 \times \ell} \stackrel{\circ R^{\prime \prime}}{\longrightarrow} \mathcal{K}^{1 \times \ell^{\prime \prime}}, \\
\mathcal{K}^{k^{\prime}} \stackrel{R^{\prime} \circ}{\longleftarrow} \mathcal{K}^{k} \stackrel{R \circ}{\longleftarrow} \mathcal{K}^{k^{\prime}} \stackrel{R^{\prime \prime} \circ}{\longleftarrow} \mathcal{K}^{\ell^{\prime}}
\end{gathered}
$$

are exact. Moreover $R^{\prime}$ and $R^{\prime \prime}$ also satisfy the rank conditions

$$
\forall z \in \mathbb{C}: \operatorname{rank}\left(R^{\prime}(z)\right)=\operatorname{rank}\left(R^{\prime}\right), \operatorname{rank}\left(R^{\prime \prime}(z)\right)=\operatorname{rank}\left(R^{\prime \prime}\right) .
$$

Due to Cor. 4.8 the sequence

$$
\begin{gathered}
\mathcal{P E}^{\ell^{\prime \prime}} \stackrel{R^{\prime \prime} \circ}{\longrightarrow} \mathcal{P} \mathcal{E}^{\ell} \stackrel{R \circ}{\longrightarrow} \mathcal{P} \mathcal{E}^{k} \stackrel{R^{\prime} \circ}{\longrightarrow} \mathcal{P} \mathcal{E}^{k} \text { is exact and } \\
\operatorname{cl}_{E}\left(R^{\prime \prime} \circ \mathcal{E}^{\ell^{\prime \prime}}\right)=\left\{w \in \mathcal{E}^{\ell} ; R \circ w=0\right\}, \operatorname{cl}_{E}\left(R \circ \mathcal{E}^{\ell}\right)=\left\{w \in \mathcal{E}^{k} ; R^{\prime} \circ w=0\right\}
\end{gathered}
$$

are behaviors.

Proof. Only (112) has to be shown. The first exact sequence in (110) implies the exact sequence

$$
\mathcal{E}_{\mathfrak{m}_{E}(z)}^{\prime 1 \times k^{\prime}} \stackrel{\circ R^{\prime}}{\longrightarrow} \mathcal{E}_{\mathfrak{m}_{E}(z)}^{\prime 1 \times k} \stackrel{\circ R}{\longrightarrow} \mathcal{E}_{\mathfrak{m}_{E}(z)}^{\prime 1 \times \ell} \stackrel{\circ R^{\prime \prime}}{\longrightarrow} \mathcal{E}_{\mathfrak{m}_{E}(z)}^{\prime 1 \times \ell} R^{\prime \prime} \rightarrow 0 .
$$

This is a free resolution of the free module $\mathcal{E}_{\mathfrak{m}_{E}(z)}^{\prime 1 \times \ell} R^{\prime \prime}$ and is therefore split exact. This property is preserved by application of any additive functor. We use $\mathbb{C}=\mathcal{E}^{\prime} / \mathfrak{m}_{E}(z)=$ $\mathcal{E}_{\mathfrak{m}_{E}(z)}^{\prime} / \mathcal{E}_{\mathfrak{m}_{E}(z)}^{\prime} \mathfrak{m}_{E}(z)$, apply the functor $N \mapsto \mathbb{C} \otimes_{\mathcal{E}_{\mathfrak{m}_{E}(z)}^{\prime}}^{\prime} N$ to this sequence and obtain the exact sequence

$$
\mathbb{C}^{1 \times k^{\prime}} \stackrel{\circ R^{\prime}(z)}{\longrightarrow} \mathbb{C}^{1 \times k} \stackrel{\circ R(z)}{\longrightarrow} \mathbb{C}^{1 \times \ell} \longrightarrow \mathbb{C} \otimes_{\mathcal{E}_{\mathfrak{m}_{E}(z)}^{\prime}}\left(\mathcal{E}_{\mathfrak{m}_{E}(z)}^{\prime 1 \times \ell} R^{\prime \prime}\right) \rightarrow 0 .
$$

This, in turn, implies

$$
\operatorname{rank}\left(R^{\prime}(z)\right)=k-\operatorname{rank}(R(z))=k-\operatorname{rank}(R)=\operatorname{rank}\left(R^{\prime}\right) .
$$

The same argument applied to the second exact sequence in (110) furnishes $\operatorname{rank}\left(R^{\prime \prime}(z)\right)=$ $\operatorname{rank}\left(R^{\prime \prime}\right)$ for all $z \in \mathbb{C}$.

If $\mathcal{K} U=\mathcal{K}^{1 \times p}\left(\operatorname{id}_{p},-H\right)$ is any IO structure then $\mathcal{B}:=U^{\perp}$ is weakly controllable (cf. Thm. 6.1,(i), ) if and only if

$$
U=\mathcal{E}^{\prime 1 \times(p+m)} \bigcap \mathcal{K}^{1 \times p}\left(\mathrm{id}_{p},-H\right)=\left\{(\xi, \eta) \in \mathcal{E}^{\prime 1 \times(p+m)} ; \xi H+\eta=0\right\} .
$$

Conversely if $H \in \mathcal{K}^{p \times m}$ is any matrix then

$$
U:=\mathcal{E}^{\prime 1 \times(p+m)} \bigcap \mathcal{K}^{1 \times p}\left(\operatorname{id}_{p},-H\right)=\left\{(\xi, \eta) \in \mathcal{E}^{\prime \times(p+m)} ; \xi H+\eta=0\right\}
$$

is closed. The gen. beh. $\mathcal{B}:=U^{\perp}$ is the only weakly controllable IO gen. beh. with transfer matrix $H$ and is called the weakly controllable realization of $H$. If

$$
\begin{aligned}
& H D=N \text { where } D \in \mathcal{E}^{\prime m \times m^{\prime}}, N \in \mathcal{E}^{\prime p \times m^{\prime}}, \operatorname{rank}(D)=m \text { then } \\
& U:=\operatorname{ker}\left(\circ\left(\begin{array}{c}
N \\
D
\end{array}\right)^{-1}\right)=\left(\circ\left(\begin{array}{c}
N \\
D
\end{array}\right)\right)^{-1}(0)=\left\{(\xi, \eta) \in \mathcal{E}^{\prime 1 \times(p+m)} ; \xi N+\eta D=0\right\} \\
& \text { and } \mathcal{B}=U^{\perp} \underset{(46)}{=} \mathrm{cl}_{E}\left(\mathcal{B}_{c}\right) \text { where } \\
& \mathcal{B}_{c}:=\left(\begin{array}{l}
N \\
D
\end{array}\right) \circ \mathcal{E}^{m^{\prime}}=\left\{\left(\begin{array}{l}
y \\
u
\end{array}\right) \in \mathcal{E}^{p+m} ; \exists x \in \mathcal{E}^{m^{\prime}}:\left(\begin{array}{l}
y \\
u
\end{array}\right)=\left(\begin{array}{l}
N \circ x \\
D \circ x
\end{array}\right) \cdot\right\}
\end{aligned}
$$

The standard representation of this type is $H=N D^{-1}$ with $m^{\prime}=m$ and $\operatorname{det}(D) \neq 0$. 
Corollary 7.11. Assume a representation (117) of $H \in \mathcal{K}^{p \times m}$ such that $\operatorname{rank}\left(\begin{array}{c}N(z) \\ D(z)\end{array}\right)=$ $m$ for all $z \in \mathbb{C}$. Then the weakly controllable realization $\mathcal{B}$ of $H$ is an IO behavior $\mathcal{B}=\left\{\left(\begin{array}{c}y \\ u\end{array}\right) \in \mathcal{E}^{p+m} ; P \circ y=Q \circ u\right\}$ with $P H=Q$ and $\operatorname{rank}(P)=p$ and, of course (cf. Thm. 6.1,(v)), $p=\operatorname{rank}(P)=\operatorname{rank}(P(z),-Q(z))$ for all $z \in \mathbb{C}$.

Proof. From Result 7.9 and Cor. 7.10 we derive the existence of a matrix $(P,-Q) \in$ $\mathcal{E}^{\prime k \times(p+m)}$ and exact sequences

$$
\mathcal{K}^{1 \times k} \stackrel{\circ(P,-Q)}{\longrightarrow} \mathcal{K}^{1 \times(p+m)} \stackrel{\circ \stackrel{(N}{D})}{\longrightarrow} \mathcal{K}^{1 \times m^{\prime}} \text { and } \mathcal{P} \mathcal{E}^{m^{\prime}} \stackrel{\left(\begin{array}{l}
D \\
D
\end{array}\right) \circ}{\longrightarrow} \mathcal{P} \mathcal{E}^{p+m} \stackrel{(P,-Q) \circ}{\longrightarrow} \mathcal{P} \mathcal{E}^{k} .
$$

Then

$$
\mathcal{B}=U^{\perp}=\operatorname{cl}_{E}\left(\left(\begin{array}{l}
N \\
D
\end{array}\right) \circ \mathcal{E}^{m^{\prime}}\right) \underset{(113)}{=}\left\{\left(\begin{array}{c}
y \\
u
\end{array}\right) \in \mathcal{E}^{p+m} ; P \circ y=Q \circ u\right\}
$$

is indeed a behavior. Moreover

$$
\begin{gathered}
(P,-Q)\left(\begin{array}{l}
N \\
D
\end{array}\right)=0 \Longrightarrow Q D=P N=P H D \underset{\operatorname{rank}(D)}{\Longrightarrow} Q m=P H \Longrightarrow \\
\operatorname{rank}(P)=\operatorname{rank}(P,-Q)=p+m-\operatorname{rank}\left(\begin{array}{l}
N \\
D
\end{array}\right)=p+m-m=p .
\end{gathered}
$$

Theorem 7.12. Assume that $\mathcal{B}=\left\{\left(\begin{array}{c}y \\ u\end{array}\right) \in \mathcal{E}^{p+m} ; P \circ y=Q \circ u\right\}$ is the weakly controllable realization of $H \in \mathcal{K}^{p \times m}$ and a behavior, hence

$$
P H=Q \text { and } \operatorname{rank}(P)=\operatorname{rank}(P,-Q)=\operatorname{rank}(P(z),-Q(z)) \text { for all } z \in \mathbb{C} \text {. }
$$

Then there is a matrix $\left(\begin{array}{l}N \\ D\end{array}\right) \in \mathcal{E}^{\prime(p+m) \times m^{\prime}}$ such that

$$
H D=N \text { and } \forall z \in \mathbb{C}: m=\operatorname{rank}(D)=\operatorname{rank}\left(\begin{array}{c}
N \\
D
\end{array}\right)=\left(\begin{array}{c}
N(z) \\
D(z)
\end{array}\right) .
$$

Hence the weakly controllable realization of $H$ is a behavior if and only if a matrix $\left(\begin{array}{l}N \\ D\end{array}\right)$ with the properties of Cor. 7.11 exists.

Proof. Result 7.9 and Cor. 7.10 furnish a matrix $\left(\begin{array}{l}N \\ D\end{array}\right) \in \mathcal{E}^{\prime(p+m) \times m^{\prime}}$ such that

$$
\begin{aligned}
& \mathcal{K}^{1 \times k} \stackrel{\circ(P,-Q)}{\longrightarrow} \mathcal{K}^{1 \times(p+m)} \stackrel{\circ\left(\begin{array}{l}
N \\
D
\end{array}\right)}{\longrightarrow} \mathcal{K}^{1 \times m^{\prime}} \text { is exact and } \\
& \forall z \in \mathbb{C}: \operatorname{rank}\left(\begin{array}{l}
N \\
D
\end{array}\right)=\operatorname{rank}\left(\begin{array}{c}
N(z) \\
D(z)
\end{array}\right) .
\end{aligned}
$$

We conclude

$$
\begin{gathered}
P N=Q D=P H D \underset{\operatorname{rank}(P)=p}{\Longrightarrow} N=H D \text { and } \\
\operatorname{rank}(D)=\operatorname{rank}\left(\begin{array}{l}
N \\
D
\end{array}\right)=p+m-\operatorname{rank}(P,-Q)=m .
\end{gathered}
$$

For the proof of the next theorem we consider $n \in \mathbb{N}$ and the nondegenerate bilinear form

$$
\mathcal{K}^{1 \times n} \times \mathcal{K}^{n} \rightarrow \mathcal{K},(\xi, x) \mapsto \xi x=\sum_{i=1}^{n} \xi_{i} x_{i}
$$


For subsets $V \subseteq \mathcal{K}^{1 \times n}$ resp. $X \subseteq \mathcal{K}^{n}$ we define the polar spaces

$$
V^{\circ}:=\left\{x \in \mathcal{K}^{n} ; V x=0\right\}, X^{\circ}:=\left\{\xi \in \mathcal{K}^{1 \times n} ; \xi X=0\right\} .
$$

If $V$ and $X$ are $\mathcal{K}$-subspaces then $V=V^{\circ \circ}$ and $X=X^{\circ \circ}$.

For the data from (115) we have

$$
\begin{gathered}
U^{\circ}=(\mathcal{K} U)^{\circ}=\left(\mathcal{K}^{1 \times p}\left(\operatorname{id}_{p},-H\right)\right)^{\circ}=\left(\begin{array}{c}
H \\
\operatorname{id}_{m}
\end{array}\right) \mathcal{K}^{m}, \\
\mathcal{K} U=\mathcal{K}^{1 \times p}\left(\operatorname{id}_{p},-H\right)=\left(\left(\underset{\operatorname{id}_{m}}{H}\right) \mathcal{K}^{m}\right)^{\circ} .
\end{gathered}
$$

Theorem 7.13. 1. If $m=1$ or $p=1$, in particular in the SISO case $p=m=1$, the unique weakly controllable realization $U^{\perp}$ of a transfer matrix $H \in \mathcal{K}^{p \times m}$ is a behavior where $U:=\left\{(\xi, \eta) \in \mathcal{E}^{\prime 1 \times(p+m)} ; \xi H+\eta=0\right\}$.

Proof. We use the notations from (115).

1.(i) Assume $m=1, p \geq 1, n:=p+1$. Choose a nonzero common denominator $D$ of $H$ and define

$$
\begin{gathered}
0 \neq f=\left(f_{1}, \cdots, f_{n}\right)^{\top}:=\left(\begin{array}{c}
H \\
1
\end{array}\right) D=\left(\begin{array}{c}
H D \\
D
\end{array}\right) \in \mathcal{E}^{\prime n}, \text { hence } U=\mathcal{E}^{\prime \times n} \bigcap(f \mathcal{K})^{\circ}, \\
0 \neq f_{0}:=\prod\left\{f_{i} ; 1 \leq i \leq n, f_{i} \neq 0\right\} \in \mathcal{E}^{\prime}, V:=V_{\mathbb{C}}\left(f_{0}\right) \supseteq V_{\mathbb{C}}\left(f_{i}\right) \text { if } f_{i} \neq 0, \\
\forall 1 \leq i \leq n \text { with } f_{i} \neq 0 \forall z \in V: k_{i}(z):=\operatorname{mult}\left(f_{i}, z\right), \\
k(z):=\min \left\{k_{i}(z) ; 1 \leq i \leq n, f_{i} \neq 0\right\} \leq k_{i}(z) \text { if } f_{i} \neq 0 .
\end{gathered}
$$

In the sequel we consider $i$ with $1 \leq i \leq n$ and $f_{i} \neq 0$ only. From the multiplicities we derive, for all $z \in V$, the representations

$$
f_{i}=(s-z)^{k_{i}(z)} f_{i}^{z},(s-z)^{-k(z)} f_{i}=(s-z)^{k_{i}(z)-k(z)} f_{i}^{z}, f_{i}^{z} \in \mathcal{E}^{\prime}, f_{i}^{z}(z) \neq 0 .
$$

The $f_{i}$ are contained in $\mathcal{E}^{\prime}$ and hence in some $\mathcal{O}_{a, p}$ (cf. (18)). We infer

$$
\forall z \in V: f_{i}^{z}=(s-z)^{-k_{i}(z)} f_{i},(s-z)^{-k(z)} f_{i}=(s-z)^{k_{i}(z)-k(z)} f_{i}^{z} \in \mathcal{O}_{a, p} .
$$

We choose numbers $a(z)>0, z \in V$, such that

$$
\begin{gathered}
\forall i \text { with } 1 \leq i \leq n \text { and } f_{i} \neq 0: \sum_{z \in V} a(z)\left\|(s-z)^{-k(z)} f_{i}\right\|_{a, p}<\infty \Longrightarrow \\
g_{i}:=\sum_{z \in V} a(z)(s-z)^{-k(z)} f_{i} \in \mathcal{O}_{a, p} \subset \mathcal{E}^{\prime} \Longrightarrow \forall z_{0} \in V: \\
g_{i}=a\left(z_{0}\right)\left(s-z_{0}\right)^{k_{i}\left(z_{0}\right)-k\left(z_{0}\right)} f_{i}^{z_{0}}+\sum_{z_{0} \neq z \in V} a(z)(s-z)^{-k(z)} f_{i}
\end{gathered}
$$

For each $z_{0} \in V$ we may and do choose an index $i$ such that

$$
\begin{gathered}
k_{i}\left(z_{0}\right)=k\left(z_{0}\right) \text { since } k\left(z_{0}\right)=\min \left\{k_{i}\left(z_{0}\right) ; 1 \leq i \leq n, f_{i} \neq 0\right\} \\
\Longrightarrow g_{i}=a\left(z_{0}\right) f_{i}^{z_{0}}+\sum_{z_{0} \neq z \in V} a(z)(s-z)^{-k(z)} f_{i}, f_{i} \neq 0 \Longrightarrow \\
g_{i}\left(z_{0}\right)=a\left(z_{0}\right) f_{i}^{z_{0}}\left(z_{0}\right)+\sum_{z_{0} \neq z \in V} a(z)\left(z_{0}-z\right)^{-k(z)} f_{i}\left(z_{0}\right), a\left(z_{0}\right) f_{i}^{z_{0}}\left(z_{0}\right) \neq 0 .
\end{gathered}
$$


If $k_{i}\left(z_{0}\right)=\operatorname{mult}\left(f_{i}, z_{0}\right)=0$ then $f_{i}\left(z_{0}\right) \neq 0$. If $k_{i}\left(z_{0}\right)>0$ then $f_{i}\left(z_{0}\right)=0$ and the last equation in (121) implies $g_{i}\left(z_{0}\right)=a\left(z_{0}\right) f_{i}^{z_{0}}\left(z_{0}\right) \neq 0$. We conclude that for all $z_{0} \in V$ there is an index $i$ such that $f_{i}\left(z_{0}\right) \neq 0$ or $g_{i}\left(z_{0}\right) \neq 0$. Define $g_{i}:=0$ if $f_{i}=0$, $g:=\left(g_{1}, \cdots, g_{n}\right)^{\top}$ and $R:=(f, g) \in \mathcal{E}^{\prime n \times 2}$. For this matrix we have just shown that for all $z_{0} \in V$ either $f\left(z_{0}\right) \neq 0$ or $g\left(z_{0}\right) \neq 0$. The vector $f=\left(\begin{array}{c}H_{D} D \\ D\end{array}\right), D \neq 0$, is nonzero and hence $f\left(z_{0}\right) \neq 0$ for all $z_{0} \notin V=V_{\mathbb{C}}\left(f_{0}\right)$. We infer that

$$
\operatorname{rank}\left(R\left(z_{0}\right)\right) \geq 1 \text { for all } z_{0} \in \mathbb{C} .
$$

(ii) Recall $g_{i}=\sum_{z \in V} a(z)(s-z)^{-k(z)} f_{i}$. For all $\xi \in U:=\mathcal{E}^{\prime 1 \times n} \bigcap(f \mathcal{K})^{\circ}$ we get

$$
\begin{gathered}
\xi f=\sum_{i=1}^{n} \xi_{i} f_{i}=0 \Longrightarrow \sum_{i=1}^{n} \xi_{i} g_{i}=\sum_{z \in V} a(z)(s-z)^{-k(z)} \sum_{i=1}^{n} \xi_{i} f_{i}=0 \Longrightarrow \\
\xi g=0 \Longrightarrow \xi R=0 \Longrightarrow(f \mathcal{K})^{\circ}=\mathcal{K} U \subseteq\left(R \mathcal{K}^{2}\right)^{\circ} \subseteq(f \mathcal{K})^{\circ} \Longrightarrow \\
\left(R \mathcal{K}^{2}\right)^{\circ}=(f \mathcal{K})^{\circ} \Longrightarrow R \mathcal{K}^{2}=f \mathcal{K}=\left(\begin{array}{c}
H \\
1
\end{array}\right) \mathcal{K} \Longrightarrow \operatorname{rank}(R)=\operatorname{rank}(f)=1 \Longrightarrow \\
\forall z \in \mathbb{C}: 1=\operatorname{rank}(R)=\operatorname{rank}(R(z)) \text { and } U=\mathcal{E}^{\prime 1 \times n} \bigcap\left(R \mathcal{K}^{2}\right)^{\circ} .
\end{gathered}
$$

With these data Cor. 7.11 implies that $\mathcal{B}=U^{\perp}$ is a behavior.

2. Assume $p:=1, m \geq 1$ and $n:=1+m$. Then $\mathcal{K} U=\mathcal{K}(1,-H) \neq 0$. Choose any nonzero vector $f=\left(f_{1}, \cdots, f_{n}\right) \in U$, hence $\mathcal{K} U=\mathcal{K} f$. As in part 1 . we construct

$$
\begin{gathered}
g_{i}=\left\{\begin{array}{ll}
\sum_{z \in V} a(z)(s-z)^{-k(z)} f_{i} & \text { if } f_{i} \neq 0 \\
0 & \text { if } f_{i}=0
\end{array},\right. \\
g:=\left(g_{1}, \cdots, g_{n}\right), R:=\left(\begin{array}{l}
f \\
g
\end{array}\right) \in \mathcal{E}^{2 \times(1+m)} \text { such that } \\
\left(\begin{array}{c}
H \\
\mathrm{id}_{m}
\end{array}\right) \mathcal{K}^{m}=(\mathcal{K}(1,-H))^{\circ}=U^{\circ}=\left(\mathcal{K}^{1 \times 2} R\right)^{\circ}, \mathcal{K} U=\mathcal{K}(1,-H)=\mathcal{K}^{1 \times 2} R \\
\operatorname{rank}(R)=1 \text { and } \forall z \in \mathbb{C}: \operatorname{rank}(R(z))=\operatorname{rank}(R)=1 .
\end{gathered}
$$

From Result 7.9 and Cor. 7.10 we infer a matrix $R^{\prime \prime}=\left(\begin{array}{l}N \\ D\end{array}\right) \in \mathcal{E}^{\prime(1+m) \times \ell^{\prime \prime}}$ such that

$$
\begin{aligned}
& \mathcal{O}^{1 \times 2} \stackrel{\circ R}{\longrightarrow} \mathcal{O}^{1 \times(1+m)} \stackrel{\circ\left(\begin{array}{l}
N \\
D
\end{array}\right)}{\longrightarrow} \mathcal{O}^{1 \times \ell^{\prime \prime}} \text { and } \mathcal{K}^{1 \times 2} \stackrel{\circ R}{\longrightarrow} \mathcal{K}^{1 \times(1+m)} \stackrel{\circ\left(\begin{array}{l}
N \\
D
\end{array}\right)}{\longrightarrow} \mathcal{K}^{1 \times \ell^{\prime \prime}} \\
& \text { are exact and } \forall z \in \mathbb{C}: \operatorname{rank}\left(\begin{array}{c}
N(z) \\
D(z)
\end{array}\right)=\operatorname{rank}\left(\begin{array}{l}
N \\
D
\end{array}\right)=1+m-\operatorname{rank}(R)=m .
\end{aligned}
$$

We conclude

$$
\mathcal{K}(1,-H)\left(\begin{array}{l}
N \\
D
\end{array}\right)=\mathcal{K}^{1 \times 2} R(\underset{D}{N})=0 \Longrightarrow N=H D, \operatorname{rank}(D)=\operatorname{rank}\left(\begin{array}{l}
N \\
D
\end{array}\right)=m .
$$

Hence $N=H D$ is a representation as in (117) and satisfies the condition of Cor. 7.11. Therefore the weakly controllable realization of $H$ is a behavior.

\section{Concluding remarks}

We discuss several questions that the reviewer raised in her/his report.

1. The usefulness of the Fourier-Laplace transform: In Section 3 the embedding $\mathcal{E}^{\prime} \subset \mathcal{O}=\mathcal{O}(\mathbb{C})$ is explained. Algebraic properties of $\mathcal{O}$ are used at several 
instances. The Paley-Wiener-Schwartz theorem (19) and Ehrenpreis' fundamental complement (20) are essential analytic, not algebraic tools for the proof of Thms. 5.8 and 7.13. The space $\mathcal{E}$ is not an $\mathcal{O}$-module and therefore the Bézout property of $\mathcal{O}$ does not help much for subbehaviors of $\mathcal{E}^{\ell}$. Our paper [5] is devoted to ${ }_{A} W$-behaviors where both the signal space $W$ and the ring $A$ of operators consist of multivariate analytic functions.

2. The size of $\mathcal{E}^{\prime}$ : The smaller and simpler Bézout domain $\mathcal{O} \cap \mathbb{C}(s)\left[\sigma, \sigma^{-1}\right] \subset$ $\mathcal{E}^{\prime}, \sigma=e^{-i \tau s}$, is the canonical ring of operators for delay-differential (DD) equations with commensurate delays in $\mathbb{Z} \tau$ [16],[23]. The present paper is a contribution to more general convolution behaviors, for instance to those defined by DD-equations with incommensurate delays in the lattice $L=\mathbb{Z} \tau_{1} \oplus \cdots \oplus$ $\mathbb{Z} \tau_{k}, k>1$, with their associated ring of operators [17, Thm. 2.2)]

$$
\mathcal{H}_{L}:=\mathcal{O} \bigcap \mathbb{C}(s)\left[\sigma_{1}, \sigma_{1}^{-1}, \cdots, \sigma_{k}, \sigma_{k}^{-1}\right] \underset{(89)}{\subset} \mathcal{H}_{\mathcal{R}}, \sigma_{j}:=e^{-i \tau_{j} s} .
$$

In contrast to the case $k=1$ [16], [23] the algebraic, topological and constructive properties of $\mathcal{H}_{L}$ are only little known. There is no analogue of the analytic properties (19) and (20) of $\mathcal{E}^{\prime}$ for $\mathcal{H}_{\mathcal{R}}$ and $\mathcal{H}_{L}$ and therefore the study of $\mathcal{E}^{\prime}$ instead of $\mathcal{H}_{L}$ seems advantageous (cf. Cor. 5.11). Since every distribution in $f \in \mathcal{E}^{\prime}$ is the limit of finite linear combinations of point distributions $\delta_{\tau}, \tau \in \mathbb{R}$, $[31,(\mathrm{VI}, 3 ; 16)]$ the convolution $(f \circ w)(t)$ is a limit of finite sums $\sum_{i=1}^{m} a_{i} w(t+$ $\tau_{i}$ ) that have an obvious interpretation from the engineering point of view. A good property of $\mathcal{H}_{\mathcal{R}} \supseteq \mathcal{H}_{L}$ is that all its nonzero elements are invertible in the distributional sense (cf. remarks following (89)), and this was essentially used in Cor. 7.6.

3. Computations and constructions: In contrast to the theory of finitely presented modules over the Bézout domain $\mathcal{O} \cap \mathbb{C}(s)\left[\sigma, \sigma^{-1}\right]$ from above [16], [23] the theory of f.g. $\mathcal{E}^{\prime}-$ or $\mathcal{H}_{L}$-modules is presently indeed unconstructive to a wide extent, both in the present paper and its predecessors. This applies even to the Bézout domain $\mathcal{O}(\mathbb{C})$ since the convergent power series are determined by infinitely many coefficients and cannot therefore be exactly represented in a computer. Thm. 4.4 with Lemma 4.2 and Cor. 4.3 as preparations and Thms. 5.6 and 5.8 are as constructive as possible for the given data.

4. Coherence as in [28]: The coherence of the rings $\mathcal{E}^{\prime}, \mathcal{H}_{L}$ and $\mathcal{H}_{\mathcal{R}}$ is not known and does probably not hold. Coherence signifies that the intersection of two f.g. ideals is again f.g.. But f.g. ideals of $\mathcal{E}^{\prime}$ are not closed in general (cf. (22) and Cor. 5.9) and nothing is known about nonclosed ideals. In Schwartz' seminal paper ideal of $\mathcal{E}^{\prime}$ means closed ideal [30, p. 912]. The f.g. ideals of $\mathcal{O}\left(\mathbb{C}^{n}\right), n \geq 1$, are closed, but coherence of $\mathcal{O}\left(\mathbb{C}^{n}\right), n>1$, is nevertheless not known and probably not valid (cf. [5, Cor. 6.3]).

5. Algebraic Analysis, for instance [28]: If a signal module ${ }_{A} W$ of analytic or engineering interest is given this notion can refer to the theory of f.g. $A$-modules $M$, called systems by Fliess, or to that of the solution modules or behaviors $\mathcal{B}:=\operatorname{sol}_{W}(M) \underset{\text { Malgrange }}{\cong} \operatorname{Hom}_{A}(M, W)$. Unless certain injectivity properties of ${ }_{A} W$ can be derived as in the present paper the module theory (algebra) does not help very much in analytic or engineering problems concerning $\mathcal{B}$ even if the 
ring $A$ has good algebraic properties, for instance being noetherian or coherent or even Bézout.

6. Fractional representation, coprime factorization, for instance [28]: In the present paper these appear in Cor. 7.11 and Thm. 7.12 in the form $P H=Q$ or $H D=N$ if the weakly controllable realization $\mathcal{B}(H)$ of a transfer matrix $H \in \mathcal{K}^{p \times m}, \mathcal{K}:=\operatorname{quot}\left(\mathcal{E}^{\prime}\right)$, is a behavior. The coprimeness condition is $\operatorname{rank}(P(z),-Q(z))=p=\operatorname{rank}(P), z \in \mathbb{C}, \operatorname{resp} \cdot \operatorname{rank}\left(\begin{array}{c}N(z) \\ D(z)\end{array}\right)=m=$ $\operatorname{rank}(D), \quad z \in \mathbb{C}$. The existence of $(P,-Q)$, that of $\left(\begin{array}{l}N \\ D\end{array}\right)$ and the behavior property of the gen. beh. $\mathcal{B}(H)$ are equivalent, but it is open whether they always hold. Thm. 7.13 proves these properties for the cases that $p$ or $m$ are 1 . According to Cor. 7.6 they always hold if $H \in \operatorname{quot}(\mathcal{R})$ (cf. Cor. 5.10).

\section{References}

[1] C.A. Berenstein, B.A. Taylor, 'A New Look at Interpolation Theory for Entire Functions of One Variable', Advances in Mathematics 33(1979), 109-143

[2] C.A. Berenstein, B.A. Taylor, 'Mean-Peridic Functions', Internat. J. Math. and Math. Sci. 3(1980), 199-235

[3] N. Bourbaki, Topological Vector Spaces, Springer, New York, 1987

[4] H. Bourlès, U. Oberst, 'Duality for Differential-Difference Systems over Lie Groups', SIAM J. Control Optim. 48(2009), 2051-2084

[5] H. Bourlès, U. Oberst, 'Elimination, fundamental principle and duality for analytic linear systems of partial differential-difference equations with constant coefficients', Math. of Control, Signals, Systems 24(2012), 351-402

[6] H. Bourlès, U. Oberst, 'Convolution behaviors and topological algebra', Proc. MTNS 2014, 1260-1267, Groningen, 2014

[7] D. Brethé, J.J. Loiseau, 'A result that could bear fruit for the control of delaydifferential systems', Proc. 4th IEEE Mediteranean Symp. Control and Autom., 168-172, Chania, Greece, 1996

[8] J. Delsarte, 'Les fonctions moyenne-périodiques', J. Math. Pures et Appl. 14(1935), 403-453

[9] L. Ehrenpreis, 'Solutions of some problems of division III', Amer. J. Math. 78(1956), 685-715

[10] L. Ehrenpreis, 'Solutions of some problems of division IV', Amer. J. Math. 82(1960), 522-588

[11] L. Ehrenpreis, Fourier Analysis In Several Complex Variables, WileyInterscience, New York, 1970

[12] S.J.L. van Eijndhoven, L.C.G.J.M. Habets, 'Equivalence of Convolution Systems in a Behavioral Framework', Math. Control Signals Systems 16(2003), 175-206 
[13] M. Fliess, H. Mounier, 'Interpretation and comparison of various types of delay system controllabilities', pp. 330-335 in Proceedings IFAC Conference System Structure and Control, Nantes, France, July 1995

[14] O. Forster, 'Primärzerlegung in Steinschen Algebren', Math. Ann. 154(1964), 307-329

[15] H. Glüsing-Lürssen, 'A behavioral approach to delay-differential systems', SIAM J. Control Optim. 35(1997), 480-499

[16] H. Glüsing-Lürssen, Linear Delay-Differential Systems with Commensurate Delays: An Algebraic Approach, Lecture Notes in Mathematics 1770, Springer, Berlin, 2002

[17] H. Glüsing-Lürssen, P. Vettori, S. Zampieri, 'The algebraic structure of DD systems: a behavioral perspective', Kybernetica 37(2001), 397-426

[18] L.C. Habets, 'System equivalence for AR-systems over rings-With an application to delay-differential systems', Math. Contr. Sign. Syst. 12(1999), 219-244

[19] L. Hörmander, The Analysis of Linear Partial Differential Operators I, Springer, Berlin, 1983

[20] L. Hörmander, The Analysis of Linear Partial Differential Operators II, Springer, Berlin, 1983

[21] J.-P. Kahane, 'Sur quelques problèmes d'unicité et de prolongement, relatifs aux fonctions approchables par de sommes d'exponentielles', Ann. Inst. Fourier 5(1953/54), 39-130

[22] A.F. Leont'ev, 'Differential-difference equations', Mat. Sb. 24(1949), 347-374

[23] J.-J. Loiseau, 'Algebraic tools for the control and stabilization of time-delay systems', IFAC Reviews, Annual Reviews in Control 24(2000), 135-149

[24] H. Matsumura, Commutative ring theory, Cambridge University Press, Cambridge, 1986

[25] H. Mounier, 'Algebraic interpretations of the spectral controllability of a linear delay system', Forum Math. 10(1998), 39-58

[26] U. Oberst, 'Variations on the Fundamental Principle for Linear Systems of Partial Differential and Difference Equations with Constant Coefficients', Applicable Algebra in Engineering, Communication and Computing 6(1995), 211-243

[27] F. Parreau, Y. Weit, 'Schwartz's theorem on mean-periodic vector-valued functions', Bull. Soc. Math. France 117(1989), 319-325

[28] A. Quadrat, 'The fractional representation approach to synthesis problems: An algebraic analysis viewpoint. Part I: (Weakly) doubly coprime factorizations', SIAM J. Control Optim. 42(2003), 266-299

[29] P.M. Rocha and J.C. Willems, 'Behavioral controllability of delay-differential systems', SIAM J. Control Optim. 35(1997), 254-264 
[30] L. Schwartz, 'Théorie générale des fonctions moyenne-périodiques', Ann. Math. 48(1947), 857-929

[31] L. Schwartz, Théorie des distributions, Hermann, Paris, 1966

[32] P. Vettori, S. Zampieri, 'Controllability of Systems Described by Convolutional or Delay-Differential Equations', SIAM J. Control Optim. 39(2000), 728-756.

[33] P. Vettori, S. Zampieri, 'Some Results on Systems Described by Convolution Equations', IEEE Trans. Autom. Control 46(2001), 793-797

[34] P. Vettori, S. Zampieri, ' Module theoretic approach to controllability of convolutional systems', Linear Alg. and Appl. 351/352(2002), 739-759

[35] P. Vettori, S. Zampieri, 'Stability and stabilizability of delay-differential systems', pp. 307-322 in B. Hanzon, M. Hazewinkel (Eds.), Constructive Algebra and Systems Theory, Royal Netherlands Academy of Arts and Sciences, 2006

[36] Y. Yamamoto, 'Pseudo-rational input/output maps and their realizations: A fractional representation approach to infinite-dimensional systems', SIAM J. Control Optim. 26(1988), 1415-1430

[37] Y. Yamamoto, J.C. Willems, 'Behavioral Controllability and Coprimeness for a Class of Infinite-Dimensional Systems', Proceedings of the 47th Conference on Decision and Control, Cancun, 2008, 1513-1518 